\title{
THE ONSET OF VORTEX TURBULENCE
}

\author{
Greg Huber ${ }^{1}$ \\ Center for Polyme Studies \\ Boston University \\ Boston, MA 02215 \\ and \\ Lawrence Berkeley Laboratory \\ University of California \\ Berkeley, CA 94720 U.S.A.
}

Ph.D. Thesis

December 1992

\footnotetext{
${ }^{1}$ Supported in part by a DARPA-NASA fellowship in parallel processing, administered by the Institute for Advanced Computer Studies, University of Maryland, and in part by the Applied Mathematical Sciences Subprogram of the Office of Energy Research, U.S. Department of Energy under Contract DE-AC(13-76SF(00098.
} 


\section{Contents}

1 The Complex Ginzburg-Landau Equation 1

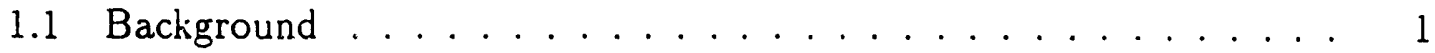

1.2 Near the Hopf bifurcation . . . . . . . . . . . . . . . . 4

1.2.1 Taylor expansions .................. 4

1.2.2 Rescaling space and time .............. 8

1.2.3 Amplitude equation ................. 9

1.3 Coupled-map formulation ................ 10

1.3.1 Basic idea ...................... 10

1.3.2 Timescales................... 11

1.3.3 Nonlocal map .................... 12

1.3 .4 Local map ..................... 13

1.4 Homogeneous solution .................. 13

1.4.1 Linear stability .................. 13

1.4.2 Benjamin-Feir and $\alpha-\beta$ space ........... 15

1.4.3 The Brusselator .................. 17

2 Vortex States $\quad 20$

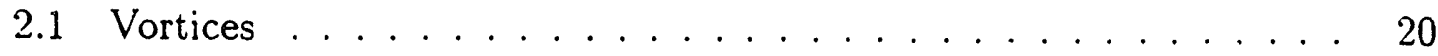


2.1 .1 Defects.................... 20

2.1.2 Topological charge and boundary conditions . . . . . 22

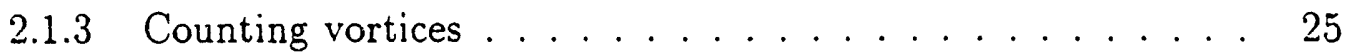

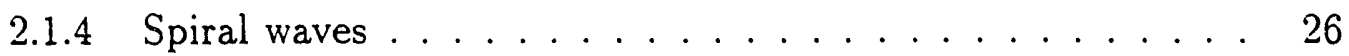

2.2 Frozen States . . . . . . . . . . . . . . . . . . . . . 29

2.2 .1 Initial conditions $\ldots \ldots \ldots \ldots \ldots \ldots$

2.2 .2 Amplitude portraits $\ldots \ldots \ldots \ldots \ldots$

2.2 .3 Wavelength charts $\ldots \ldots \ldots \ldots \ldots \ldots$

2.3 Turbulent States $\ldots \ldots \ldots \ldots \ldots \ldots \ldots$

2.4 Transition Line $\ldots \ldots \ldots \ldots \ldots \ldots$

3 Vortex Density and Decay 45

3.1 Density Traces . . . . . . . . . . . . . . . . 45

3.2 Initial Decay . . . . . . . . . . . . . . . . . 48

3.3 Slow decay to Frozen State . . . . . . . . . . . . . . . 51

3.4 Fast Decay to Frozen State . . . . . . . . . . . . . 51

3.4.1 Parabola law: Numerics . . . . . . . . . . . . . . . 51

3.4 .2 Nucleation . . . . . . . . . . . . . . . 55

3.4.3 Parabola law: Model . . . . . . . . . . . . . . . 6 60

$3.5 \quad$ XY-type Decay . . . . . . . . . . . . . . . . . . 62

3.5 .1 The exponent $\xi \ldots \ldots \ldots \ldots \ldots \ldots \ldots \ldots$

3.5.2 Annihilation scale . . . . . . . . . . . . . 67

3.5.3 Continuous ordering $\ldots \ldots \ldots \ldots 67 \ldots \ldots$

3.5 .4 Interaction volume $\ldots \ldots \ldots \ldots \ldots \ldots \ldots$

3.5.5 Freely-decaying turbulence . . . . . . . . . . . . . 72 
4 Transient Turbulence $\quad \mathbf{7 4}$

4.1 Transient Overview . . . . . . . . . . . . . . 74

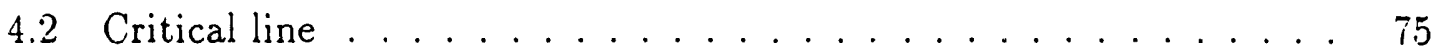

4.2.1 Local density minimum. . . . . . . . . . . 75

4.2.2 Linear stability analysis . . . . . . . . . . . 75

4.3 Near the Transition Line . . . . . . . . . . . . . . 78

4.3.1 Absolute instability . . . . . . . . . . . . . . 78

4.3.2 Nucleation time . . . . . . . . . . . . . . . 79

4.4 Geometrical Nucleation . . . . . . . . . . . . . . 80

4.4 .1 Outer perturbations ............... 80

4.4 .2 Divergence.................... 82

5 Conclusions and Extensions $\quad \mathbf{8 4}$

5.1 Conclusions ....................... 84

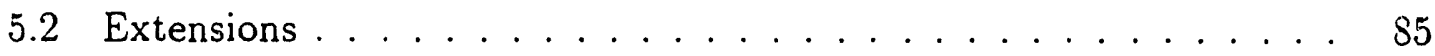

5.2 .1 Suprises in quadrant III .............. 85

5.2 .2 Vortex lines in three dimensions . . . . . . . . . 88 


\section{Chapter 1}

\section{The Complex Ginzburg-Landau Equation}

\section{$1.1 \quad$ Background}

It is the goal of this thesis to investigate some of the unusual and spectacular properties near the transition to turbulence in a two-dimensional field of limit-cycle oscillators. Of particular interest are the dynamics of topological defects (vortices) associated with the onset of turbulence.

The complex Ginzburg-Landau equation describes an extended reaction-diffusion system close to the bifurcation of a steady state into a stable, periodic orbit. In the jargon of nonlinear dynamics, it is the amplitude equation corresponding to a Hopf bifurcation. Because of the generality of the assumptions under w'lich it is derived, the complex Ginzburg-Landau equation describes systems in contexts other than chemical reactions with diffusion. Examples include Rayleigh-Bénard convection and the phase fields of multimode lasers. The reaction-diffusion model 
is, however, a sufficiently general model to frame our discussion.

A reaction-diffusion system, strictly speaking, is a set of ordinary differential equations coupled by diffusion terms. In the continuum limit, one speaks of a reaction-diffusion field, where the total system is viewed as a large number of identical, local systems diffusively coupled. Oscillating chemical reactions, like the Belousov-Zhabotinskii reaction $[1,2,3,4,5]$, are prime examples - even a small, spatially-isolated part of the solution is observed to oscillate in a well-defined limit cycle. An analogy to statistical mechanical models of critical phenomena is tempting, especially to the Landau picture of a coarse-grained local order parameter. However, there are important differences. In the reaction-diffusion oscillator field, small regions can persist in states far from equilibrium, thereby forming spatiotemporal patterns. Such patterns include waves, kinks, vortices, domain walls, cellular structures, and percolation clusters of activation $[6,7,8]$. Turbulence, i.e. rapid and irregular spatial and temporal dynamics, is another dramatic example of the wide range of order-disorder phenomena exhibited by these excitable media.

Let us start with a general nonlinear reaction-diffusion equation

$$
\frac{\partial \mathbf{X}}{\partial t}=\mathbf{f}(\mathbf{X}, \mu)+\mathbf{D} \nabla^{2} \mathbf{X}
$$

where $\mu$ is a tunable control parameter, and $\mathbf{D}$ a matrix of diffusion constants (often diagonal). In the case of a chemical reaction, $\mathbf{X}$ is a set of chemical concentrations, and $\mu$ the rate of inflowing chemicals. As a function of $\mu$, the system may move from a homogeneous time-independent state to a homogeneous time-periodic state beyond a critical value $\mu_{c}$. Figure 1.1 displays the generic phase portraits below and above $\mu_{c}$. The development of a stable periodic orbit from a stable steady state of an evolution equation is called a Hopf bifurcation, after a famous theorem of E. Hopf 


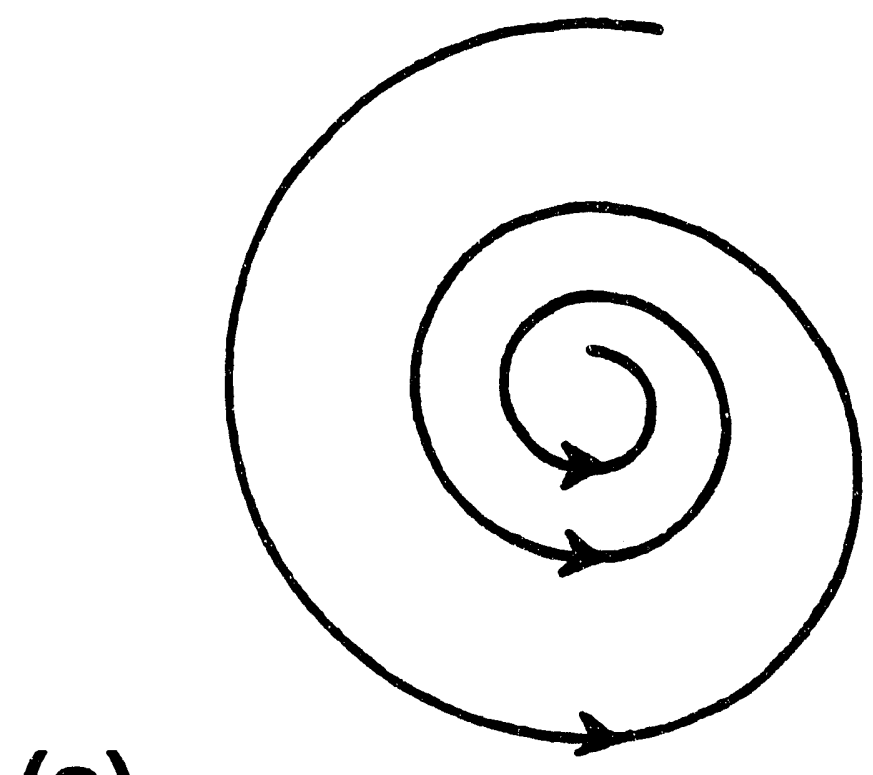

(a)

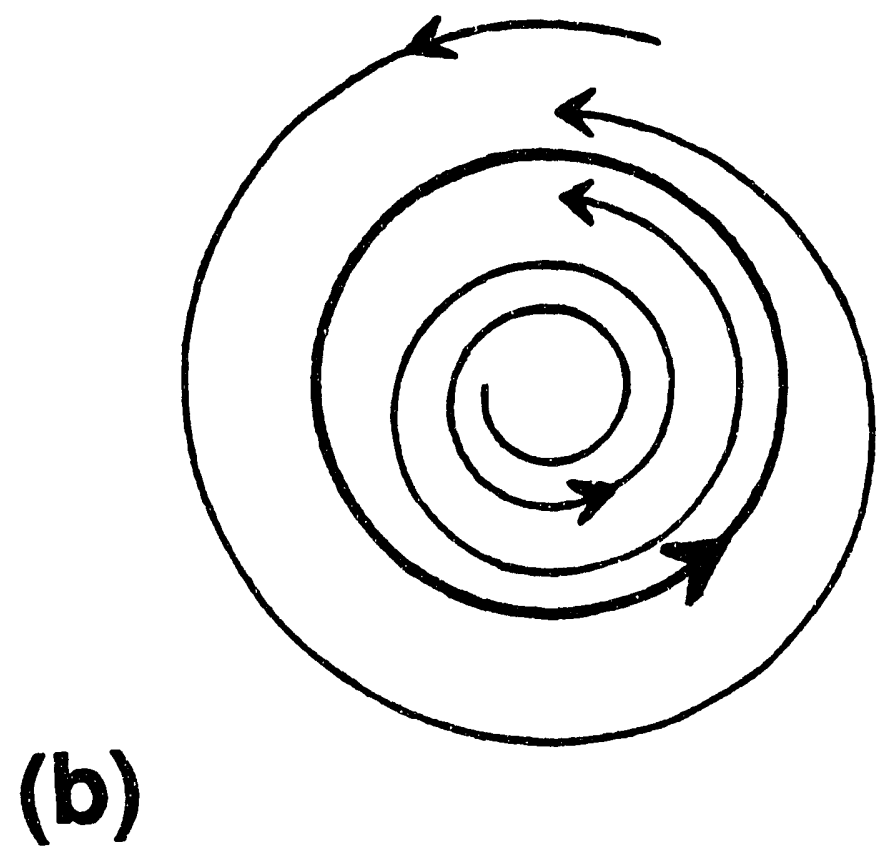

Figure 1.1: Typical subcritical (a) and supercritical (b) phase trajectories. 
[9] guaranteeing the existence of a one-parameter family of stable, periodic solutions under a set of fairly general conditions. Generically, $\mu$ can be used to parametrize this family of periodic orbits [10]. In the present study, we concentrate on the order and disorder in a field of these limit-cycle oscillators. Of course, over the space of all oscillator fields, many properties of the dynamics vary from system to system; nevertheless, there exists a universal behavior close to the Hopf bifurcation. The universal equation is called the complex Ginzburg-Landau equation, after its similarity to the order parameter equation of superconductivity and superfluidity.

\subsection{Near the Hopf bifurcation}

\subsubsection{Taylor expansions}

Let $\mathrm{X}_{0}(\mu)$ be a stationary, homogeneous solution of $(1.1)$,

$$
\mathbf{f}\left(\mathbf{X}_{0}, \mu\right)=0
$$

The expansion of (1.1) in a Taylor series, in terms of the deviation from the steady state, $\mathbf{u}(\mathbf{r}, t)=\mathbf{X}-\mathbf{X}_{\mathbf{0}}$, reads

$$
\frac{\partial \mathbf{u}}{\partial t}=\left(\mathbf{L}+\mathbf{D} \nabla^{2}\right) \mathbf{u}+\text { h.o.t. }
$$

where $\mathbf{L}$ is the Jacobian $\partial \mathbf{f}\left(\mathbf{X}_{\mathbf{0}}\right) / \partial \mathbf{X}$. Of the higher-order terms in (1.3), the first two are (using the summation convention)

$$
\begin{aligned}
\text { Muu } & =\frac{1}{2} \frac{\partial^{2} \mathbf{f}\left(\mathbf{X}_{0}\right)}{\partial X_{j} \partial X_{k}} u_{j} u_{k} \\
\text { Nuuu } & =\frac{1}{6} \frac{\partial^{3} \mathbf{f}\left(\mathbf{X}_{0}\right)}{\partial X_{j} \partial X_{k} \partial X_{l}} u_{j} u_{k} u_{l} .
\end{aligned}
$$

For the moment, we ignore the spatial degrees of freedom coming from the Laplacian in (1.3). Assume, that up to $\mu_{c}=0, \mathbf{X}_{0}$ is stable to small perturbations, 
and unstable for $\mu>0$. The stability of $\mathbf{X}_{\mathbf{0}}$ depends on the configuration of eigenvalues $\lambda$ given by

$$
\mathrm{Lu}=\lambda \mathbf{u} .
$$

If any eigenvalue of $\mathbf{L}$ has a positive real part, there exist perturbations which increase exponentially as $t \rightarrow \infty$, i.e. $\mathbf{X}_{\mathbf{0}}$ becomes uristable. Our assumption is that just one eigenvalue $\lambda_{0}$, or just one pair of complex conjugate eigenvalues $\left(\lambda_{0}, \lambda_{0}^{*}\right)$, first crosses the imaginary axis, from the left half plane, when $\mu=0$. The Hopf theorem requires two hypotheses on $\mathbf{L}[10,11]$. The first Hopf assumption is that the critical $(\mu=0)$ eigenvalues are purely imaginary, that is if $\lambda=\sigma(\mu) \pm i \omega(\mu)$, then $\sigma(0)=0, \omega(0) \equiv \iota \cdot 0$. The second Hopf condition is that the two imaginary eigenvalues cross the imaginary axis with finite velocity as $\mu$ goes through zero; in other words, $\sigma^{\prime}(0)>0$. A hypothetical critical configuration of eigenvalues is plotted in Fig. 1.2.

Allowing, now, the influence of the diffusive coupling in (1.3), i.e. non-uniform perturbations, spatial modes and the influence of system size come into play. For large systems, the eigenvalue spectrum obtained from (1.3) is almost continuous. Each eigenvalue of $\mathbf{L}$ is extended into an entire branch, as shown schematically in Fig. 1.3. The range of applicability of the Hopf theorem is thus restricted to arbitrarily small neighborhoods around $\mu_{c}$. Spatial variations are therefore essential to the question of the stability of the bifurcated solution, and more generally, any reduced version of Eq. (1.3) must necessarily take into account spatial as well as temporal degrees of freedom. 


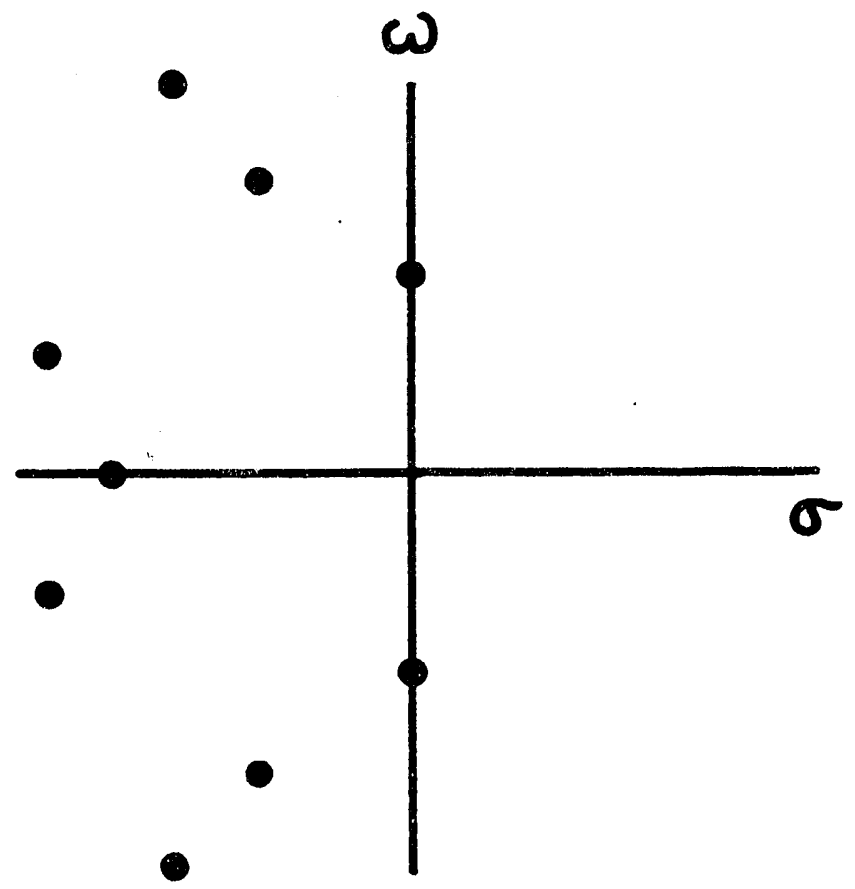

Figure 1.2: Hypothetical distribution of eigenvalues at $\mu=\mu_{c}$. 


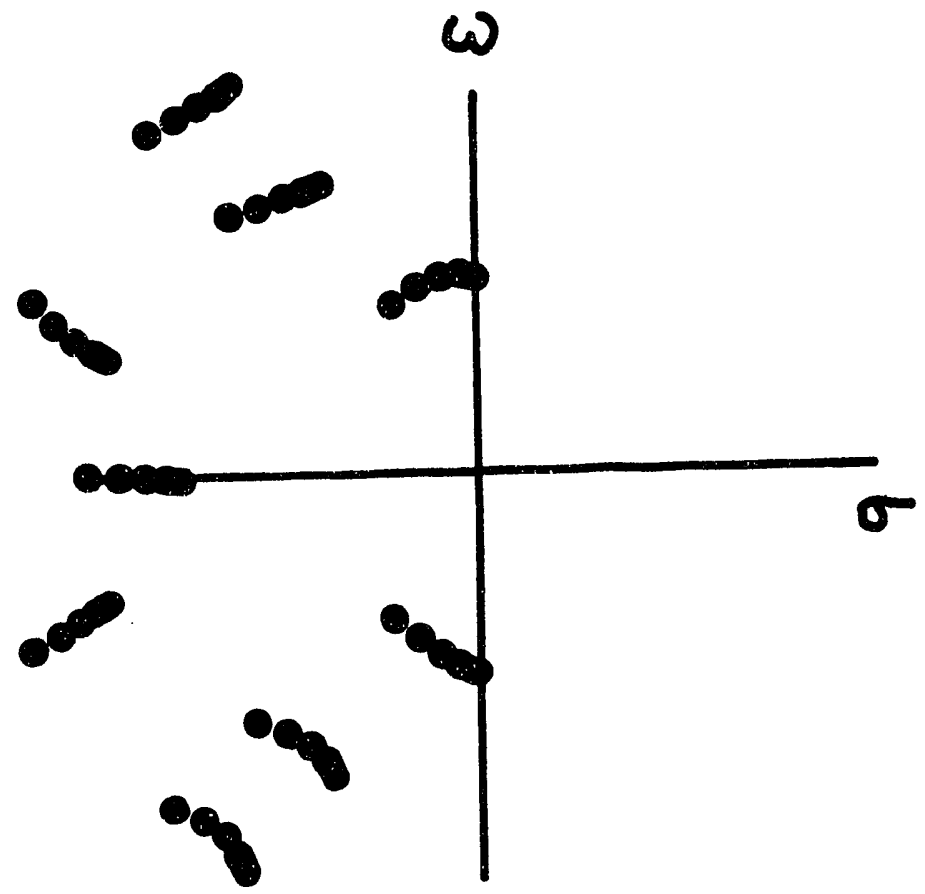

Figure 1.3: Critical distribution of eigenvalues for an extended system, in correspondence with Fig. 1.2 


\subsubsection{Rescaling space and time}

Near criticality, the Jacubian (and second and third derivatives), eigenvector and eigenvalue may be self-consistently expanded in powers of $\mu$ :

$$
\begin{aligned}
\mathbf{L} & =\mathbf{L}_{\mathbf{0}}+\mu \mathbf{L}_{1}+\mu^{2} \mathbf{L}_{2}+\cdots \\
\mathbf{M} & =\mathbf{M}_{\mathbf{0}}+\mu \mathbf{M}_{1}+\mu^{2} \mathbf{M}_{2}+\cdots \\
\mathbf{N} & =\mathbf{N}_{0}+\mu \mathbf{N}_{1}+\mu^{2} \mathbf{N}_{2}+\cdots \\
\mathbf{u} & =\mu^{1 / 2} \mathbf{u}_{1}+\mu \mathbf{u}_{2}+\mu^{3 / 2} \mathbf{u}_{3}+\cdots \\
\lambda & =\lambda_{0}+\mu \lambda_{1}+\mu^{2} \lambda_{2}+\cdots
\end{aligned}
$$

where $\lambda_{1}=\sigma_{1}+i \omega_{1}, \lambda_{2}=\sigma_{2}+i \omega_{2}$, and so on.

We now wish to derive a contracted form of (1.1), which admits slow modulations (in space and time) of the periodic orbit. In the last section, based on the nearly continuous family of eigenvalues, we argued that in the limit $\mu \rightarrow 0$ only the uniform mode survived. However, if we are dealing with an infinite system, then no neighborhood of $\pm i \omega_{0}$ is small enough to apply the Hopf ideas. Reversing the limits, by taking the system size $L$ to infinity for fixed values of $\mu$, should admit many non-uniform modes into our fixed neighborhood. On dimensional grounds, once $L$ becomes larger than $1 / \sqrt{\mu}$, a number of modes will have time scales comparable to $\omega_{0}^{-1}$, and should be treated with equal weight, since all modes in the critical neighborhood also share similar growth and decay rates.

It is clear that we must somehow "get inside" the neighborhood around the critical mode, and we do this by rescaling our space and time variables. Since $\lambda$ (a frequency) has real part of order $\mu$, time is naturally rescaled so that

$$
\tau=\mu t
$$


Allowing a slow spatial dependence of $\mathbf{u}$, rescaling of space takes the form

$$
\mathbf{s}=\sqrt{\mu} \mathbf{r} .
$$

The eigenvertor $\mathrm{u}$ can now be considered a function of the three variables $t, \tau$ and $\mathbf{s}$. Physically, this is equivalent to dealing with the long-time, long-wavelength modes in their "natural" variailes $\tau$ and $\mathrm{s}$, and reserving $t$ for the overail periodic orbit.

\subsubsection{Amplitude equation}

To first order, the substitutions in Section 1.2.2 lead to an eigenfunction in lowestharmonic form [12],

$$
\mathbf{u}_{1}(\mathrm{~s}, \tau, t)=A(\mathbf{s}, \tau) \mathbf{u}_{0} e^{\lambda_{0} t}+c . c .=A(\mathbf{s}, \tau) \mathbf{u}_{0} e^{i \omega_{0} t}+c . c .
$$

where $A(\mathrm{~s}, \tau)$ is some complex amplitude (to be determined), and $\mathbf{u}_{0}$ is the eigenvector of $\mathbf{L}_{0}$ corresponding to $\lambda_{0}$. By further matching powers of $\mu, \mathbf{u}_{2}$ can be expressed in terms of $\mathbf{u}_{1}$ and the higher-order derivatives in Eq. (1.3).

Matching the third-order terms in this way $[12,13]$, yields a closed-form expression for $A(\mathbf{s}, \tau)$, the complex Ginzburg-Landau equation,

$$
\frac{\partial A}{\partial \tau}=\lambda_{1} A-g|A|^{2} A+h \nabla^{2} A
$$

(valid in this form for $\mu>0$ ). Here the complex constants $g$ and $h$ hide some of the messy details of the intermediate calculation [13]. Explicitly,

$$
\begin{aligned}
g= & 4 \tilde{u}_{0} \mathrm{M}_{0} \mathrm{u}_{0} \mathrm{~L}_{0}{ }^{-1} \mathrm{M}_{0} \mathrm{u}_{0} \mathrm{u}_{0}{ }^{*}+ \\
& 2 \tilde{\mathbf{u}}_{0} \mathrm{M}_{0} \mathrm{u}_{0}{ }^{*}\left(\mathrm{~L}_{0}-2 i \omega_{0} \mathbf{I}\right)^{-1} \mathrm{M}_{0} \mathrm{u}_{0} \mathrm{u}_{0}-3 \tilde{\mathrm{u}}_{0} \mathrm{~N}_{0} \mathrm{u}_{0} \mathrm{u}_{0} \mathrm{u}_{0}{ }^{*} \\
h= & \tilde{\mathrm{u}}_{0} \mathrm{D} \mathbf{u}_{0},
\end{aligned}
$$


where $u_{0}{ }^{*}$ is the complex conjugate of $u_{0}$, and $\tilde{u}_{0}$ is the left eigenvertor of $\mathbf{L}_{0}$. (The operators $\mathrm{M}$ and $\mathrm{N}$ for heterogeneous operands can be read off from Eqs. (1.4,1.5).)

Without sacrificing generality, Eq. (1.15) can be further rescaled to simplify the three complex coefficients $\lambda_{1}, g$, and $h$. A scale transformation, and rotation (of A),

$$
\begin{aligned}
\mathbf{r} & =\sqrt{\sigma_{1} / \operatorname{Re}(h)} \mathbf{s}, \\
t & =\sigma_{1} \tau \\
A^{\prime} & =\sqrt{|\operatorname{Re}(g)| / \sigma_{1}} e^{-i \omega_{1} \tau} A,
\end{aligned}
$$

yields a complex Ginzburg-Landau equation of the form (dropping the prime on $A$ )

$$
\frac{\partial A}{\partial t}=A-(1+i \alpha)|A|^{2} A+(1+i \beta) \nabla^{2} A
$$

where $\alpha=\operatorname{Im}(g) / \operatorname{Re}(g)$ and $\beta=\operatorname{Im}(h) / \operatorname{Re}(h)$. This particular form clearly reveals the two-dimensional nature of the real parameter space $(\alpha, \beta)$, with $\alpha$ controlling the nonlinear term and $\beta$ the diffusional term. Henceforth, by "complex GinzburgLandau" (or CGL) we refer to this two-parameter equation (1.21), which, in our context, can be considered the simplest field of nonlinear oscillators.

\subsection{Coupled-map formulation}

\subsubsection{Basic idea}

As first noted by Oono and Puri [14], an efficient way to study Ginzburg-Landau models numerically is by simulating a lattice of dynamical maps which interact through a diffusive coupling. Such a coupled-map lattice is constructed to mimic 
a set of partial differential equations, like (1.21). Although we focus on the twodimensional square lattice in this work, the methods described here are applicable to any lattice. The idea $[15,16]$ is to integrate $(1.21)$ in two distinct operations: a local map $A_{n+1}=F\left(A_{n}\right)$ which takes care of the $A-(1+i \alpha)|A|^{2} A$ part of (1.21), and a nonlocal operator which represents the Laplacian term $(1+i \beta) \nabla^{2} A$. This nonlocal operator will be seen to be the product of an arbitrary number of Laplaceoperator terms (c.f. Section 1.3.3). The coupled-map algorithm is well-suited to the Single-Instruction Multiple-Data (SIMD) architecture of the Connection Machine 2 , and our numerical work takes full advantage of this.

\subsubsection{Timescales}

The local and nonlocal pieces are updated separately, so two distinct time steps $\tau$ and $\tau_{0}$ are introduced. To see what this separation implies for our particular form of the CGL, we write the time update for the whole equation,

$$
A_{n+1}=A_{n}+\tau_{0}\left[A_{n}-(1+i \alpha)\left|A_{n}\right|^{2} A_{n}+(1+i \beta) \nabla^{2} A_{n}\right]
$$

Reserving $\tau_{0}$ for the Laplacian term, we define a time step $\tau$ for the local terms by $\tau_{0}=a \tau(a<1)$, and simultaneously rescale $A: A_{n}=A_{n}^{\prime} / \sqrt{a}$. Dropping the prime on $A_{n}$, the result is

$$
A_{n+1}=A_{n}+\tau\left[a A_{n}-(1+i \alpha)\left|A_{n}\right|^{2} A_{n}\right]+\tau_{0}(1+i \beta) \nabla^{2} A_{n}
$$

The unit coefficient of the $A_{n}$ term in (1.22) is here rescaled by the factor $a=\tau_{0} / \tau$. The new time derivative $A_{n+1}-A_{n}$ is now taken to be $\tau$ parts local and $\tau_{0}$ parts nonlocal. Under this scheme, a rescaled "canonical" CGL equation should also have this effective coefficient. By convention, the coefficient is denoted $\mu\left(=\tau_{0} / \tau\right)$ in the 
new equation,

$$
\frac{\partial A}{\partial t}=\mu A-(1+i \alpha)|A|^{2} A+(1+i \beta) \nabla^{2} A
$$

since, like the control parameter in Section 1.2.1 its change of sign marks the location of the Hopf bifurcation. For the purposes of simulation, Eq. (1.24) replaces Eq. (1.21) as our canonical model.

\subsubsection{Nonlocal map}

We first consider the nonlocal part of (1.24), the complex heat equation

$$
\frac{\partial A}{\partial t}=(1+i \beta) \nabla^{2} A
$$

Using the time step $\tau_{0}$, we write the formal solution to (1.25),

$$
A\left(\mathbf{r}, t+\tau_{0}\right)=e^{\tau_{0}(1+i \beta) \nabla^{2}} A(\mathbf{r}, t)
$$

We approximate this solution by a nonlocal map

$$
A^{\prime}=\left(1+\frac{\tau_{0}}{m}(1+i \beta) \nabla^{2}\right)^{m} A
$$

where $\nabla^{2}$ now denotes the discrete Laplacian formed by summing over the nearest neighbors $\mathbf{r}^{\prime}$ to site $\mathbf{r}$,

$$
\nabla^{2} A(\mathbf{r}, t)=\frac{1}{4} \sum_{\mathbf{r}^{\prime}}\left(A\left(\mathbf{r}^{\prime}, t\right)-A(\mathbf{r}, t)\right)
$$

and $m$ is chosen sufficiently large. Simulational experience has shown that $m=5$ is large enough so that short-wavelength instabilities are avoided - larger values of $m$ had no appreciable effect on the observed dynamics. 


\subsubsection{Local map}

The local map $F(A)$ is constructed by omitting the Laplacian from Eq. (1.24). Let $A=R e^{i \phi}$, then the local equation becomes

$$
\begin{aligned}
& \dot{R}=\mu R-R^{3} \\
& \dot{\phi}=-\alpha R^{2} .
\end{aligned}
$$

Integration over a time interval $\tau$ renders the above as

$$
\begin{aligned}
R(t+\tau) & =\frac{\sqrt{\mu} R(t)}{\sqrt{\lambda \mu+(1-\lambda) R(t)^{2}}} \\
\phi(t+\tau) & =\phi(t)-\tau \alpha R(t)^{2}
\end{aligned}
$$

(where $\lambda=e^{-2 \mu \tau}$ ), and these comprise the map $F(A)$. The iterative coupled-map system is obtained by combining the local and nonlocal parts,

$$
A(\mathbf{r}, t+1)=F\left(A^{\prime}(\mathbf{r}, t)\right)
$$

with the parameter values $\tau=1, m=5, \tau_{0}=0.2, \mu=\tau_{0} / \tau=0.2$, which we use throughout. The lattices used are of sizes $128 \times 128$ through $1024 \times 1024$.

\subsection{Homogeneous solution}

\subsubsection{Linear stability}

The supercritical homogeneous (i.e. spatially uniform) solutions of the CGL equation (1.24), are just $A=0$ and

$$
A(t)=\sqrt{\mu} e^{-i \alpha \mu t} .
$$


The latter is Hopf's bifurcated limit cycle. While the zero solution is unstable, the Hopf theorem only guarantees the stability of (1.34) when $\mu$ is infinitesimally close to $\mu_{c}=0$. For practical uses, a finite value of $\mu$ is required; e.g. the value $\mu=0.2$ preferred in the present numerical study. To extend the analysis to finite $\mu$, it is necessary to examine the evolution of weak perturbations about the solution (1.34) in space and time. For clarity, our discussion uses the simple, canonical form (1.24), rather than the full numerical scheme based on coupled maps.

We consider weak perturbations of amplitude $(\eta)$ and phase $(0)$ of the form $[12,16]$

$$
A=(\sqrt{\mu}+\eta(\mathbf{x}, t)) e^{-i \alpha \mu t+i \theta(\mathbf{x}, t)} .
$$

Substituting (1.35) into the CGL equation (1.24), and separating the result into real and imaginary parts, yields two coupled time-evolution equations for $\eta$ and $\theta$ :

$$
\begin{aligned}
\dot{\eta}= & -2 \mu \eta+\nabla^{2} \eta-\beta(\sqrt{\mu}+\eta) \nabla^{2} 0- \\
& 2 \beta \nabla \eta \cdot \nabla \theta-3 \sqrt{\mu} \eta^{2}-(\sqrt{\mu}+\eta)|\nabla \theta|^{2}-\eta^{3} \\
(\sqrt{\mu}+\eta) \dot{\theta}= & (\sqrt{\mu}+\eta) \nabla^{2} \theta-2 \alpha \mu \eta+\beta \nabla^{2} \eta+ \\
& 2 \nabla \eta \cdot \nabla \theta-3 \alpha \sqrt{\mu} \eta^{2}-\beta(\sqrt{\mu}+\eta)|\nabla \theta|^{2}-\alpha \eta^{3} .
\end{aligned}
$$

Linearizing the above in $\eta$ and $\theta$ results in the linear equation

$$
\dot{\zeta}=\mathbf{B} \zeta
$$

with $\zeta \equiv\left(\begin{array}{c}\eta \\ \theta\end{array}\right)$ and

$$
\mathbf{B}=\left(\begin{array}{cc}
\nabla^{2}-2 \mu & -\beta \sqrt{\mu} \nabla^{2} \\
\frac{\beta}{\sqrt{\mu}} \nabla^{2}-2 \dot{a} \sqrt{\mu} & \nabla^{2}
\end{array}\right) .
$$


Transforming to momentum space leads to the eigenvalue equation

$$
\tilde{\mathbf{B}} \zeta=i \omega \zeta
$$

where

$$
\tilde{\mathbf{B}}=-\left(\begin{array}{cc}
k^{2}+2 \mu & -\beta \sqrt{\mu} k^{2} \\
\frac{\beta}{\sqrt{\mu}} k^{2}+2 \alpha \sqrt{\mu} & k^{2}
\end{array}\right) .
$$

The resulting dispersion relation reduces to

$$
\omega(k)=i\left(\mu+k^{2}\right)\left(\frac{k}{\mu}\right)^{2}(1+\alpha \beta)
$$

for small $k$. From $(1.40$ ), we see that if $\operatorname{Im}(\omega)<0$ then the small-k (longwavelength) modes are exponentially enhanced, i.e. the solution (1.34) is unstable. Likewise, for $\operatorname{Im}(\omega)>0$, the long-wavelength modes decay exponentially. The sign of the quantity $(1+\alpha \beta)$ therefore marks the instability. When

$$
1+\alpha \beta<0
$$

even the uniform limit-cycle oscillation loses its stability. This condition for longwavelength instability is known as the Benjamin-Feir stability limit [17].

\subsubsection{Benjamin-Feir and $\alpha-\beta$ space}

Exactly the same analysis can be performed for our coupled-map lattice model. The discreteness of that model results in a numerical shift in the analogous condition for instability; namely,

$$
\gamma+\alpha \beta<0
$$

where $\gamma=(1-\lambda) / 2 \mu \tau$ and $\lambda$ is defined after Eqs. $(1.31,1.32)$. Note that as $\mu \tau \rightarrow 0$, the discrete Benjamin-Feir condition (1.44) reduces to the continuum version (1.43). 


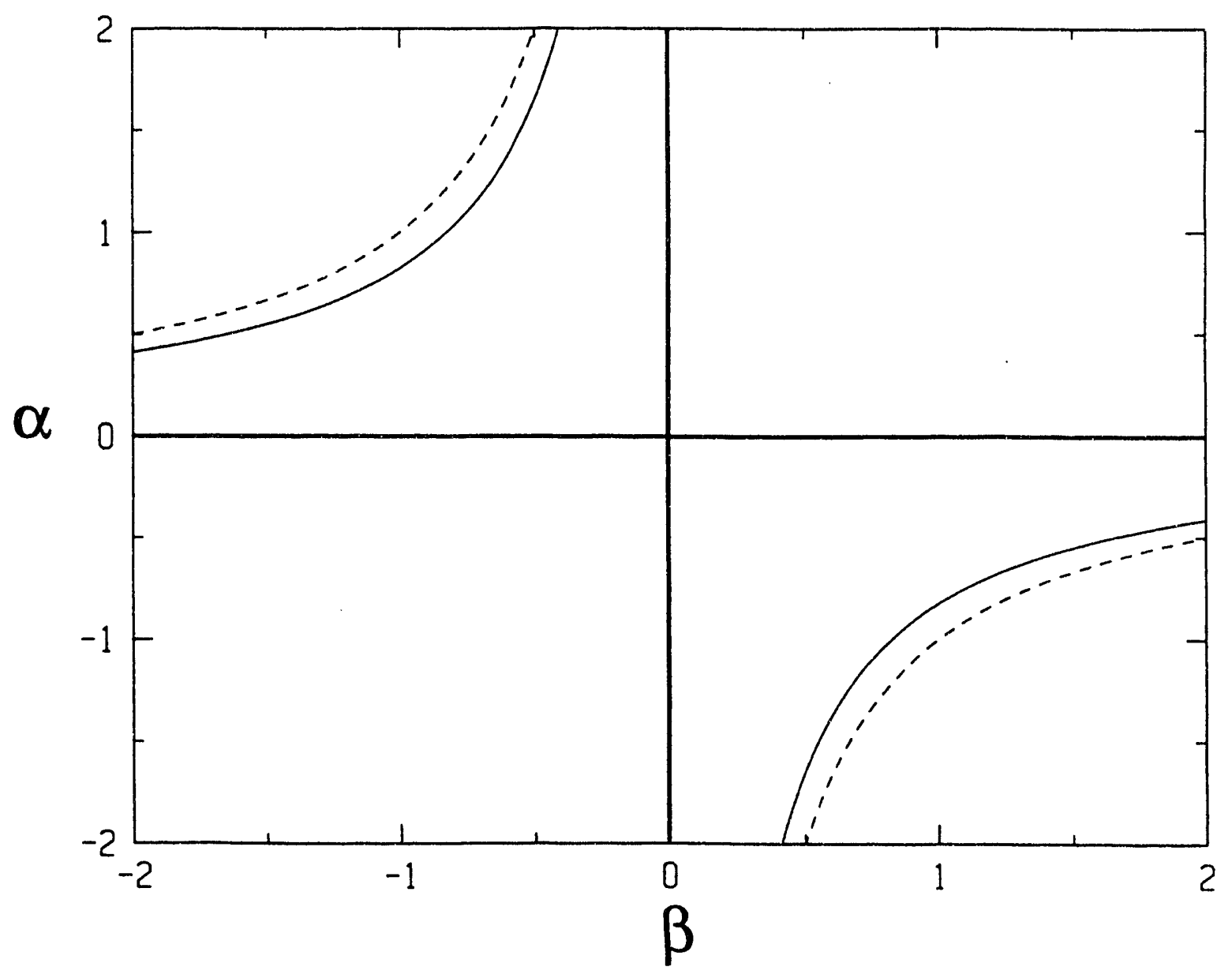

Figure 1.4: $\alpha-\beta$ phase diagram with continuum (dashed) and discrete (solid) Benjamin-Feir stability lines. 
In our simulations, with $\mu \tau=0.2$, we have $\gamma=0.8242$. If we make a plot of $\alpha$ versus $\beta$, then the hyperbola representing the Benjamin-Feir condition is shifted for the discrete case by about $18 \%$ (cf. Fig. 1.4).

The Benjamin-Feir condition has the nice symmetry of invariance under a simultaneous change of sign of $\alpha$ and $\beta$. This is not just accidental; the CGL equation (1.24) shares this symmetry. To see this, take the complex conjugate of (1.24). This will obtain a CGL equation for the field $A^{*}$, with the signs flipped on $\alpha$ and $\beta$. Only two quadrants of Fig. 1.4 are needed then. Furthermore, the stability line lies in only one of these two quadrants, the other quadrant being entirely BenjaminFeir stable. In this study, we therefore consider just quadrant II $(\alpha \geq 0, \beta \leq 0)$ of Fig. 1.4 as our phase diagram [18].

\subsubsection{The Brusselator}

In this section, we apply the analysis of Section 1.2 to a chemical reaction model known as the Brusselator $[19,20]$. Our treatment will closely follow that of references [13] and [5].

Consider the following reaction mechanism [21]:

$$
\begin{aligned}
A & \longrightarrow X \\
B+X & \longrightarrow Y+D \\
2 X+Y & \longrightarrow 3 X \\
X & \longrightarrow E .
\end{aligned}
$$

Assume that the reaction are irreversible and that the concentrations of $A$ and $B$ are kept constant. The rate equations for $X$ and $Y$ are then

$$
\dot{X}=k_{1} A-k_{2} B X+k_{3} X^{2} Y-k_{4} X
$$




$$
\dot{Y}=k_{2} B X-k_{3} X^{2} Y
$$

These can be simplified through the change of variables $\tau=k_{4} t, a=\frac{k_{1}}{k_{4}} \sqrt{\frac{k_{3}}{k_{4}}} A, b=$ $\frac{k_{2}}{k_{4}} B, x=\sqrt{\frac{k_{3}}{k_{4}}} X, y=\sqrt{\frac{k_{3}}{k_{4}}} Y$. The new rate equations, witin diffusion turned on, are then

$$
\begin{aligned}
& =a-(b+1) x+x^{2} y+D_{x} \nabla^{2} x \\
\dot{y} & =b x-x^{2} y+D_{y} \nabla^{2} y .
\end{aligned}
$$

The uniform, steady-state solution to these equations is $\left(x_{0}, y_{0}\right)=(a, b / a)$, and we define our fluctuation about this fixed point by $\mathbf{u}=(x, y)-\left(x_{0}, y_{0}\right) \equiv(\xi, \eta)$. Rewriting $(1.51,1.52)$, the time evolution of the fluctuations is given by

$$
\begin{aligned}
& \dot{\xi}=(b-1) \xi+a^{1} \eta+D_{x} \nabla^{2} \xi+f(\xi, \eta) \\
& \dot{\eta}=-b \xi-a^{2} \eta+D_{y} \nabla^{2} \eta-f(\xi, \eta)
\end{aligned}
$$

where $f(\xi, \eta)=b \xi^{2} / a+2 a \xi \eta+\xi^{2} \eta$. The above is transparently linearized, and the eigenvalues of $\mathrm{L}$ are easily computed: $\lambda=\frac{1}{2}\left(b-1-a^{2} \pm \sqrt{\left(b-1-a^{2}\right)^{2}-4 a^{2}}\right)$. Therefore, the steady state is stable iff $\operatorname{Re}(\lambda)<0$, i.e. $b<1+a^{2}$. Let us suppose that $a$ is constant, and take $b$ as our tunable control parameter $\dot{a}$ la $\mu$. Then we have the critical value $b_{c}=1+a^{2}$ marking the Hopf bifurcation. We can confirm that this is truly Hopf by computing the critical eigenvalues: $\lambda_{c}= \pm i a$.

To facilitate the small- $\mu$ expansion, we shift and normalize the control parameter to $\mu=\left(b-b_{c}\right) / b_{c}$. It is then straightforward to find the next set of operators and eigenvectors:

$$
\mathbf{L}_{\mathbf{0}}=\left(\begin{array}{cc}
a^{2} & a^{2} \\
-\left(1+a^{2}\right) & -a^{2}
\end{array}\right), \mathbf{L}_{1}=\left(1+a^{2}\right)\left(\begin{array}{cc}
1 & 0 \\
-1 & 0
\end{array}\right), \mathbf{D}=\left(\begin{array}{cc}
D_{x} & 0 \\
0 & D_{y}
\end{array}\right)
$$




$$
\mathbf{u}_{0}=\left(\begin{array}{c}
1 \\
-1+i / a
\end{array}\right), \tilde{\mathbf{u}}_{0}=\frac{1}{2}(1-i a,-i a), \lambda_{1}=\frac{1}{2}\left(1+a^{2}\right)
$$

and

$$
h=\frac{1}{2}\left[D_{x}+D_{y}-i a\left(D_{x}-D_{y}\right)\right]
$$

Likewise, the $\mathbf{M}_{\mathbf{0}}$ and $\mathbf{N}_{\mathbf{0}}$ operators can be computed to give ihe constant $g$ :

$$
g=\left[6 a+3 a^{3}+i\left(4-7 a^{2}+4 a^{4}\right)\right] / 6 a^{3} .
$$

Remembering that $\alpha=\operatorname{Im}(g) / \operatorname{Re}(g)$ and $\beta=\operatorname{Im}(h) / \operatorname{Re}(h)$, we finally arrive at the expressions

$$
\begin{aligned}
\alpha & =\frac{4-7 a^{2}+4 a^{4}}{3 a\left(a^{2}+2\right)} \\
\beta & =a \frac{D_{y}-D_{x}}{D_{y}+D_{x}}
\end{aligned}
$$

which relate our $\alpha$ and $\beta$ parameters back to the rate and diffusion constants. 


\title{
Chapter 2
}

\section{Vortex States}

\author{
IN GIRVM IMVS NOCTE \\ ET CONSVMIMVR IGNI \\ - Latin palindrome
}

\section{$2.1 \quad$ Vortices}

\subsubsection{Defects}

Totally different from the homogeneous state (1.34), are solutions that allow the field $A$ to assume phase singularities, or phase-less points. As an example of phase configurations that result in points of undefined phase, consider Fig. 2.1a. In the two-dimensional context, we refer to these defects as vortices, since the phase field undergoes rotation on a closed loop encircling one. Proceeding clockwise around the vortex in Fig. 2.1a, the field $A$ also rotates clockwise. Same for Fig. 2.1b. However, in Fig. 2.1c (or d), the field $A$ rotates counterclockwise along the same clockwise-oriented loop. These latter configurations are called antivortices. The 


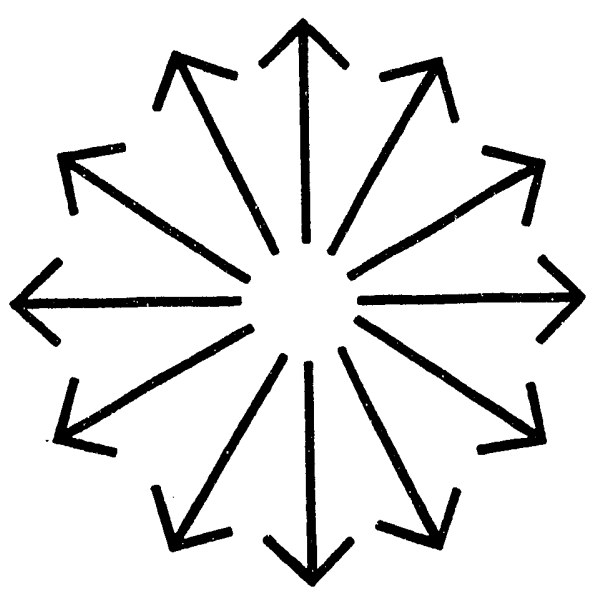

(a)

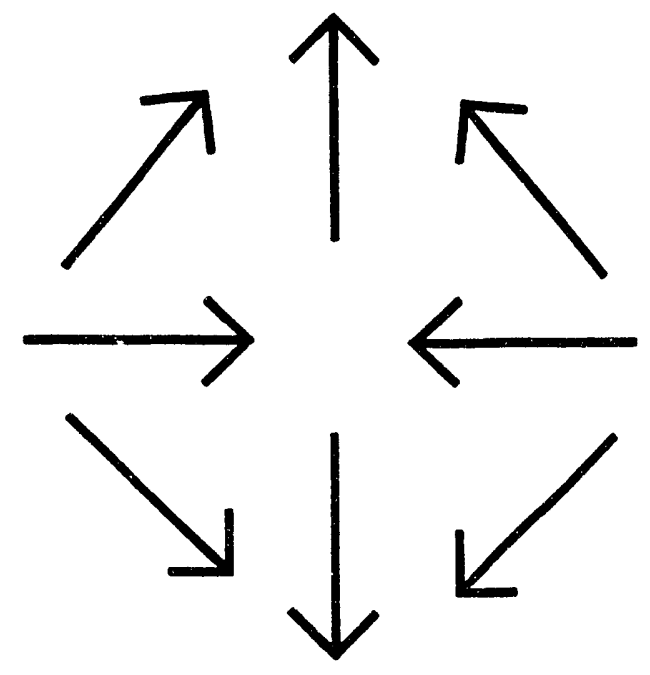

(c)

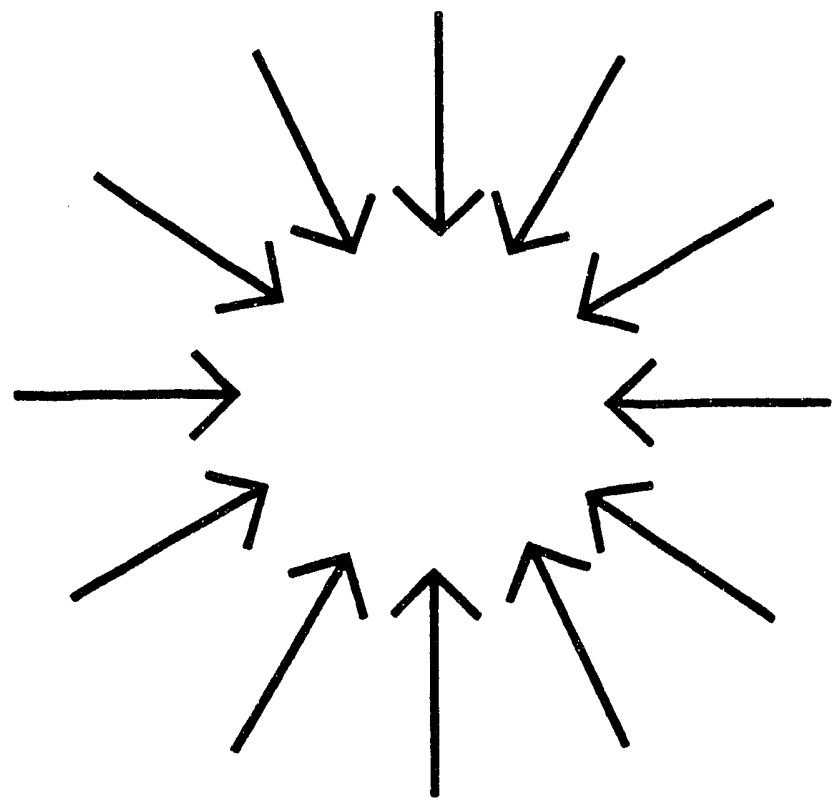

(b)
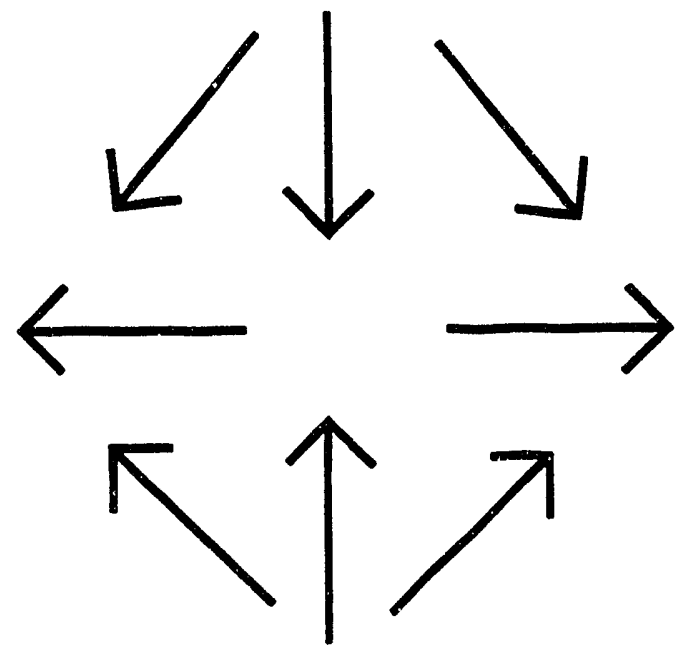

(d)

Figure 2.1: Singular phase configurations represented by unit vectors: (a) Field around a vortex of charge 1. (b) Field in (a) rotated by $\pi$. (c) Field around an antivortex of charge -1 . (d) Field in (c) rotated by $\pi$. 
phase $\phi(\mathbf{r}, t)$ at a vortex (or antivortex) center is undefined, but $|A|=0$ so that $A$ remains well-defined. (Where the distinction between vortices and antivortices is irrelevant, we refer to them collectively as vortices.) Since these defects result from the nontrivial topology of the surrounding field, they cannot simply decay away or be smoothed over. Only the merger of a vortex and antivortex can relax the field to a simpler topology.

In some respects, vortices and antivortices behave like positive and negative charges. This is best seen if the above definitions of vortices are rewritten in terms of the gradient field $\nabla \phi$. The circulation of the gradient of the phase over a closed loop containing $n_{+}$vortices and $n_{-}$antivortices is

$$
\Delta \phi=\oint \nabla \phi \cdot d l=2 \pi\left(n_{+}-n_{-}\right)
$$

Physically, this is the two-dimensional analog of Gauss' theorem from electrostatics. One interprets $n_{+}-n_{-}$as the total topological charge of $n_{+}$positive and $n_{-}$negative unit charges. (Higher integral charges are conceivable, but seemingly never appear in our simulations.) An interesting feature of this analogy is that the usual electric field lines are now isophase lines, and the usual equipotential lines are now gradient field lines (cf. Fig. 2.2). In other words, the usual electrostatic features are rotated by $\pi / 2$.

\subsubsection{Topological charge and boundary conditions}

An important global constraint on the numbers and types of vortices can be appreciated through a simple argument. It is related to the notion of "combing the hair" on a two-dimensional surface. First, picture the phase field as a field of unit vectors lying on a sphere. It is clear that the presence of a source and sink (as in 


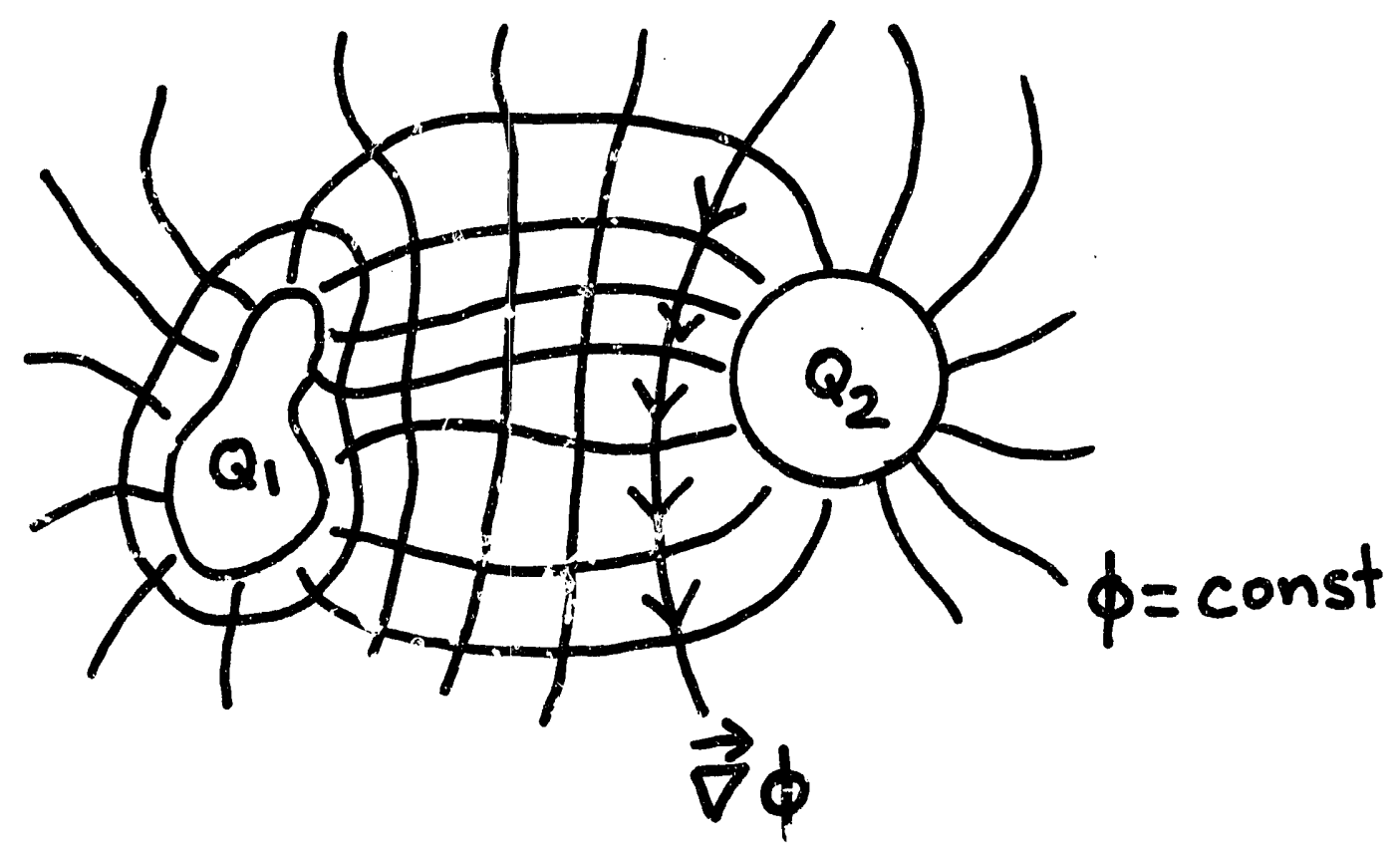

Figure 2.2: Two regions (positive and negative) of topological charge. Isophase lines $(\phi=$ constunt $)$ and gradient field $(\nabla \phi)$ are indicated. 


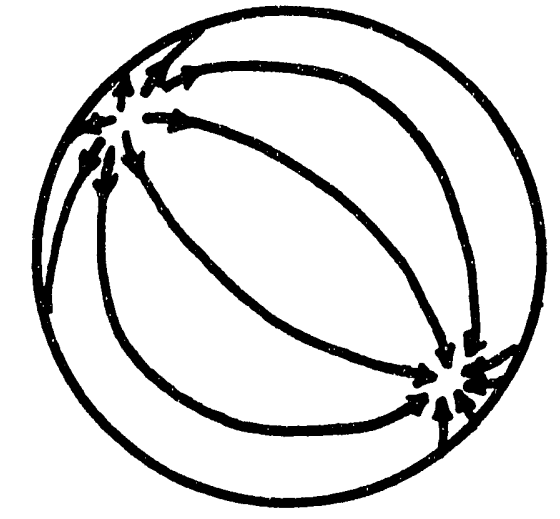

(a)

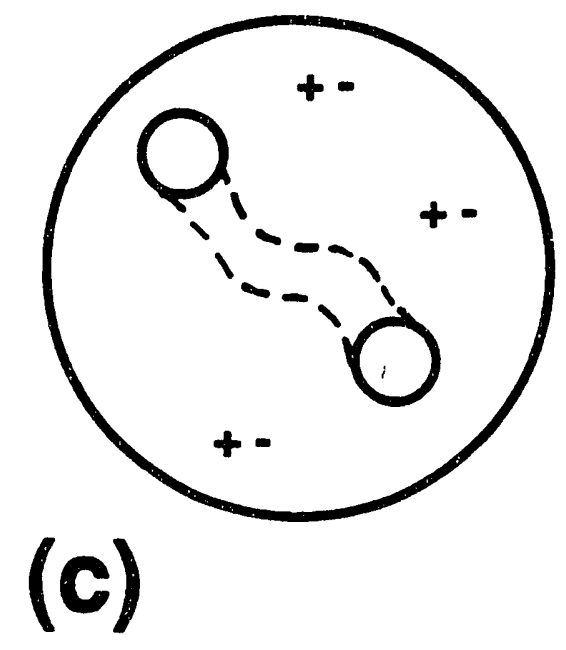

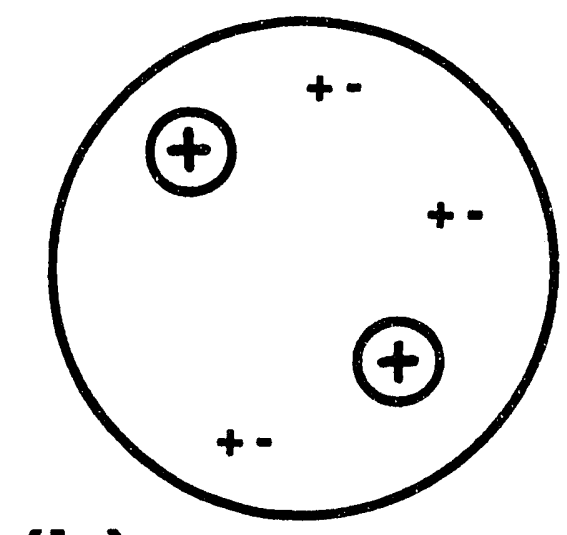

(b)

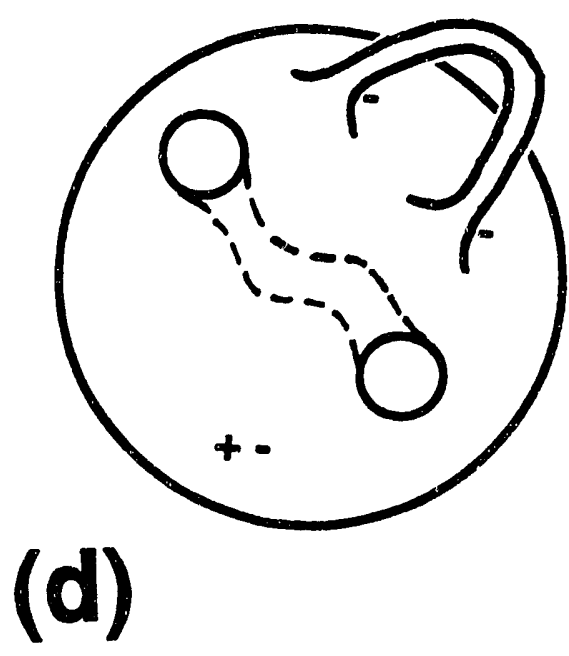

Figure 2.3: (a) Source, $\operatorname{sink}$ and $(\cos \phi, \sin \phi)$ lines on a sphere. (b) Topological charges replace source and sink. (c) Handle replaces two positive charges. (d) A subsequent handle likerates two negative charges. 
Fig. $2.1 \mathrm{a}, \mathrm{b}$ ) on the sphere leads to an otherwise continuous vector field (Fig. $2.3 \mathrm{a}$ ). In addition to this vortex-vortex pair, any number of vortex-antivortex pairs may be present, perturbing but not breaking the original field lines. These can then be separated and moved around at will. So much for the sphere - it can thus support a net topological charge of +2 (Fig. $2.3 \mathrm{~b}$ ).

Imagine now making a torus by adding a handle to the sphere, with the handle ends attached at the locations of the original vortex-vortex pair. These two vortices are no longer needed as the handle surface can smoothly carry the flow from source to sink (Fig. $2.3 \mathrm{c}$ ). The torus therefore supports a net zero charge. Likewise, the attachment of any new two-vortex handle, and the subsequent unbinding of two vortex-antivortex pairs, leads to the liberation of two antivortices - a decrease of 2 in net charge (Fig. $2.3 \mathrm{~d}$ ). Therefore, a surface with $g$ handles (i.e. a surface of genus $g$ ) supports a net charge of $2+g \times(-2)$, which is exactly the Euler characteristic $\chi$ for a compact, orientable surface.

In this study, the use of periodic boundary conditions is topologically equivalent to embedding in a toroidal surface $(g=1)$, so the relevant constraint on the total topological charge is $Q=2-2 g=0$, as stated above. For systems where unitcharged vortices and antivortices are the only defects, this guarantees equal numbers of each: $n_{+}=n_{-}$.

\subsubsection{Counting vortices}

A simple method can be used to count the vortices and antivortices in the system. We take the counterclockwise circulation about all the elementary plaquettes in the 


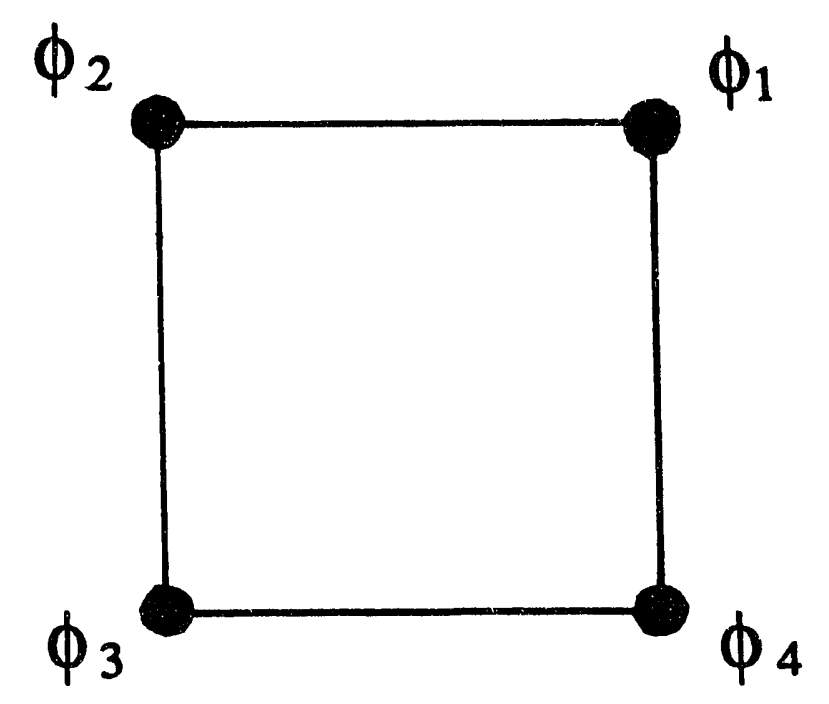

Figure 2.4: Elementary plaquette with phase variables.

lattice, using the discrete form of $(2.1)$ :

$$
Q_{p}=\frac{1}{2 \pi}\left[f\left(\phi_{4}-\phi_{3}\right)+f\left(\phi_{3}-\phi_{2}\right)+f\left(\phi_{2}-\phi_{1}\right)+f\left(\phi_{1}-\phi_{4}\right)\right]
$$

where $\phi_{i} \in[-\pi, \pi]$ and $f$ is a $2 \pi$-periodic function, with $f(x)=x$ in the dumain $|x|<\pi$.

In practice, the amplitude $|A|$ is always slightly larger than zero on our discrete lattice, and therefore no phase on a plaquette is ever truly undefined. From the definition in (2.2), it is easy to see that the possible cases are $Q_{p}=0, \pm 1$. The total number of vortices plus antivortices is then the sum of $\left|Q_{p}\right|$ over the entire lattice.

\subsubsection{Spiral waves}

Having dealt with the topological properties of point defects, we now turn to the geometry of actual vortices. Since an analytic solution describing the vortices does 


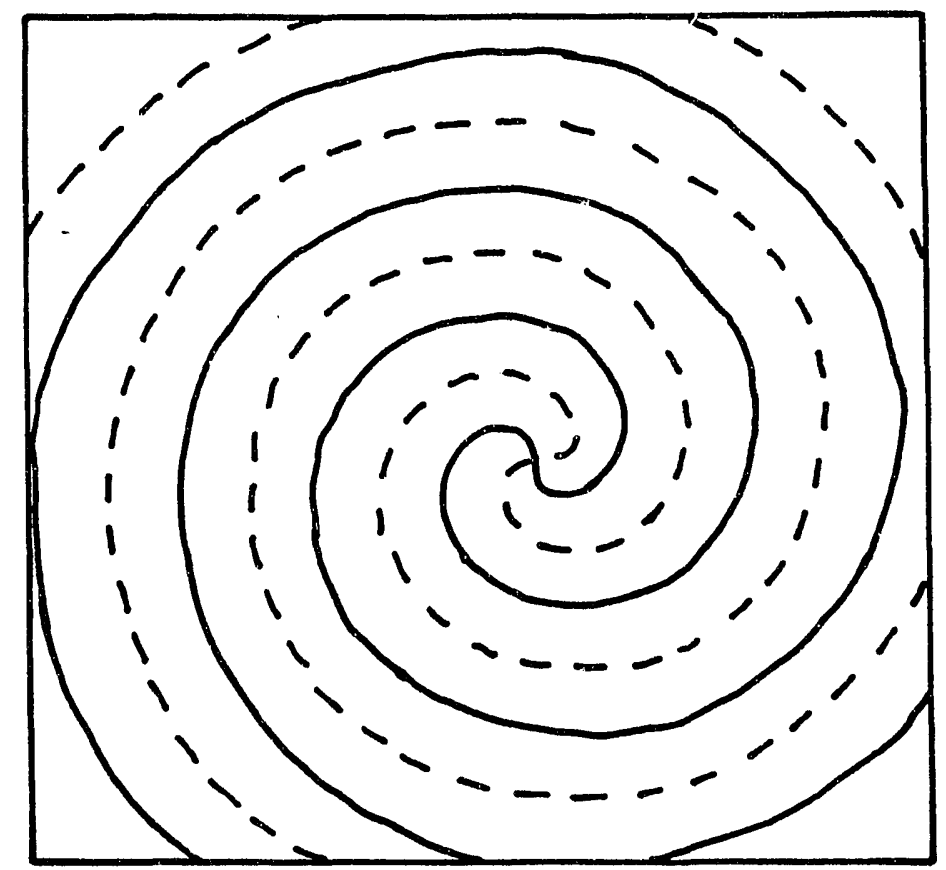

Figure 2.5: Contours of $\operatorname{Re}(A)=0$ and $\operatorname{Im}(A)=0$ for a vortex solution.

not exist [13], we begin with information gleaned from numerical experience [15, $22,23,24]$.

Generally speaking, the actual solutions resemble our schematics (Figs. 2.1) only near the vortex center. The general case has the geometry of Fig. 2.5, where the curves $\operatorname{Re}(A)=0$ and $\operatorname{Im}(A)=0$ form tightly-wound, Archimedean spirals. (The two lines must meet at the vortex core, where $A=0$.) A more complete view of the spiral-wave structure can be had from a phase portrait, using a color map extending from dark blue to dark red, corresponding to the phase range $-\pi$ to $\pi$, respectively. Fig. 2.6 shows a vortex in a system with parameters $\alpha=0.72, \beta=$ -1. Experimental observations of concentration waves in the Belousov-Zhabotinskii reaction show remarkably similar features to the phase plot in Fig. 2.6a. $[5,25]$. The corresponding amplitude plot (Fig. 2.6b), shows how the amplitude goes to zero at the vortex core, and flattens out far from the core. Here the color map ranges from 

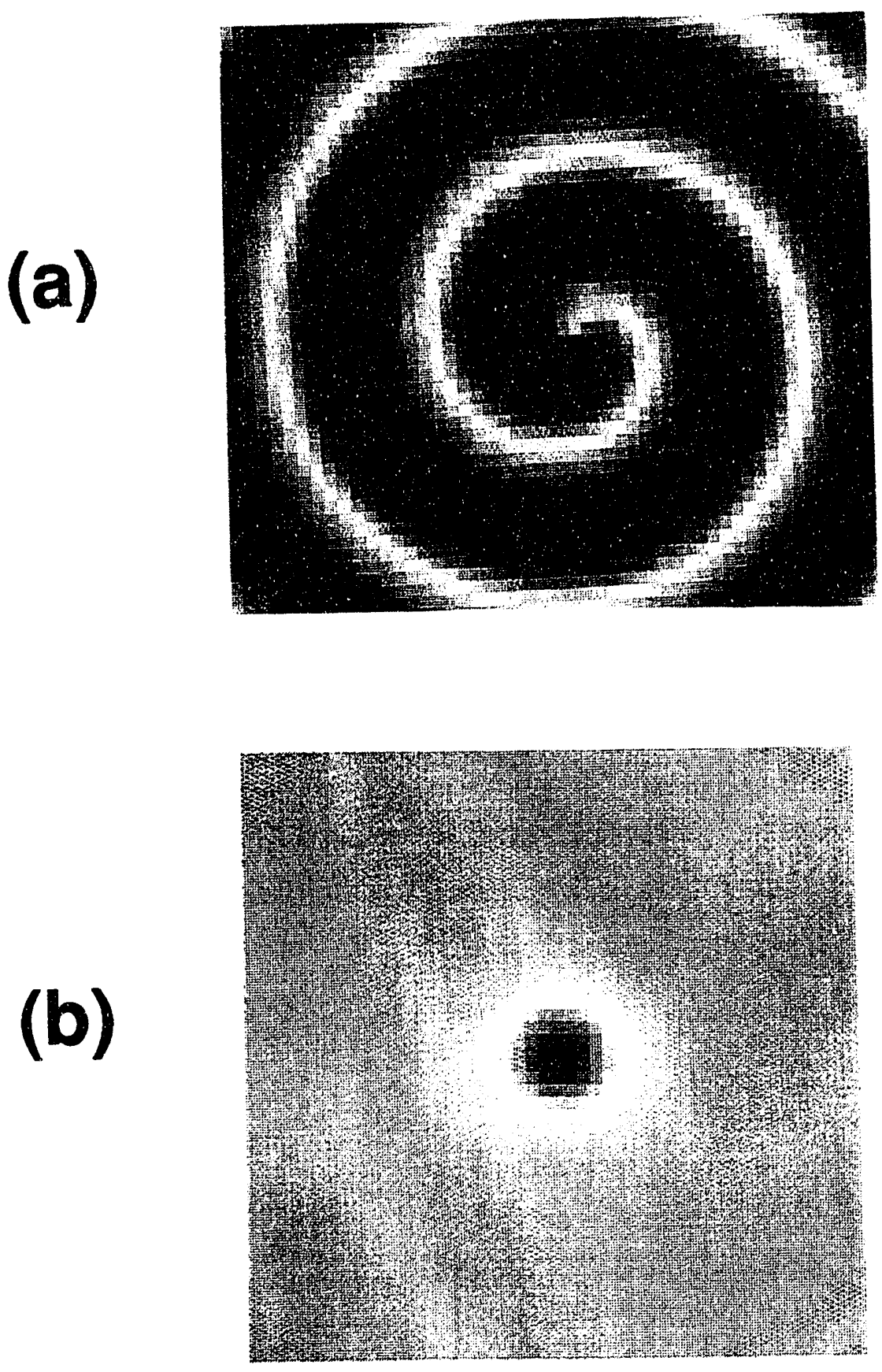

Figure 2.6: Vortex in (a) phase and (b) amplitude portraits. Pictured is a $50 \times 50$ section of a $128 \times 128$ system. 
dark blue at the amplitude minimum (zero), to dark red at the amplitude maximum (not shown). Aside from its intrinsic worth, the advantage or the disadvantage (depending on one's point of view) of the amplitude portrait is the great reduction in amount of visual information it presents. The absolute phase is lost, but this loss is compensated for by the clearly pictured localization of the vortex core. For many-vortex states, we find that plots of the amplitude are indispensable for our qualitative and quantitative arguments.

The steady rotation of spirals, seen numerically as well as experimentally, lends the vortex solutions their wave character. Rotation of the spiral pattern, at a frequency $\Omega$, can be expressed by a phase function of simplified argument: $\phi(r, \theta, t)=$ $\phi(r, \theta \pm \Omega t)$. This, with our earlier observation that $\phi(r, \theta+2 \pi, t)=\phi(r, \theta, t) \pm 2 \pi$, and together a long argument [13], implies that $\phi$ can be approximated asymptotically by

$$
\phi(r, \theta, t) \approx c_{1} r+c_{2} \log r \pm \theta+\Omega t
$$

Far from the vortex core (at $r=0$ ), the field $A$ is well approximated by a radial plane wave of constant $|A|$ and linearly increasing $\phi$. We will find this characterization particularly useful in Section 4.2.2.

\section{$2.2 \quad$ Frozen States}

\subsubsection{Initial conditions}

Here we begin a discussion of the many-vortex states, both ordered and disordered, generated by the CGL equation. Our initial conditions of choice are uniformallydistributed, random values of amplitude and phase. Amplitude is chosen from the 
interval $[0,1]$, and phase from the interval $[-\pi, \pi]$.

Starting from these random initial conditions, and depending on the parameters $\alpha$ and $\beta$, a state populated by a low density of stationary vortices and antivortices is eventually reached. The existence of this "frozen" state is based almost entirely on numerical observations $[15,18]$. (Note that it is the amplitude which is effectively frozen here; a spiral wave rotates around each vortex core.) Elphick and Meron [26] found solutions consisting of a bound pair of vortices for $\beta<0$. We find these stationary, frozen states even for $\beta=0$, suggesting the need for an intuitive, physical argument supporting them. From the local point of view, the general situation is one of two annihilating waves, of the same wavelength and frequency, colliding and unable to grow further into each others territory. Another perspective comes from thinking of the vortices as interacting point particles, whose interactions are screened by the spatial and temporal rotation of the surrounding phase field.

\subsubsection{Amplitude portraits}

As a graphic catalog of the different varieties of frozen vortex states, we perform the following simple exercise: Let a large $(512 \times 512)$ system evolve to a late time $\left(t=10^{4}\right)$, and take a snapshot of the amplitude at that time. We make two passes through the $\alpha-\beta$ phase diagram, one horizontal (fixed $\alpha$ ), and one vertical (fixed $\beta$ ). The parameter values for these two slices are $\alpha=0.5$ (Figs. 2.7,2.8) and $\beta=-1$ (Figs. 2.9,2.10).

The appearance of "domain" walls separating the spiral waves is a striking and unexpected discovery. As is clear from the amplitude plots, the walls are the global amplitude maxima of the field $A$. However, unlike the vortices, which are amplitude minima, the walls are not topological in origin. 

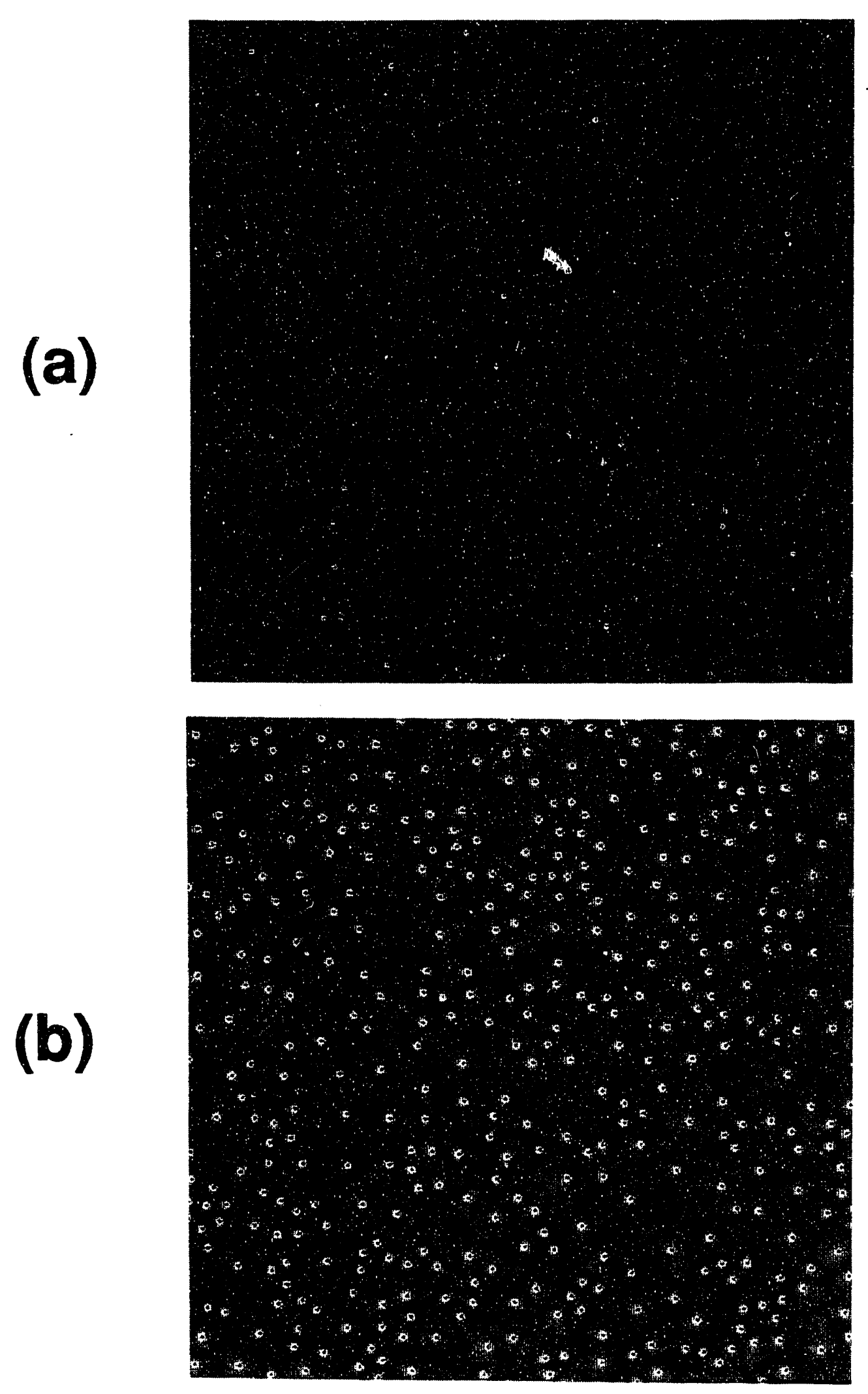

Figure 2.7: Amplitude for (a) $\beta=0$, (b) $\beta=-0.5$. $(\alpha=0.5$.) 

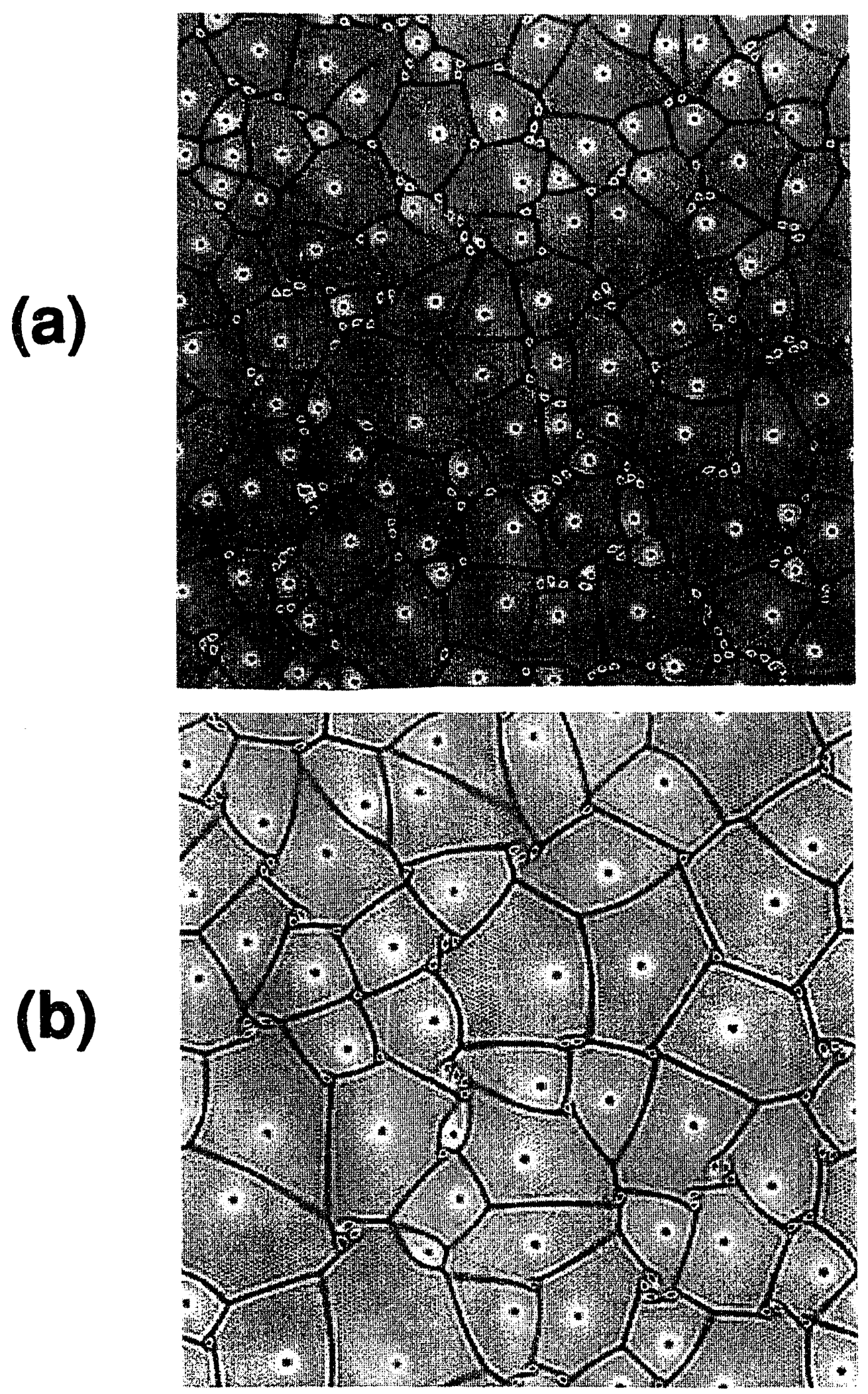

Figure 2.8: Amplitude for (a) $\beta=-1$, (b) $\beta=-1.5$. $(\alpha=0.5$.) 

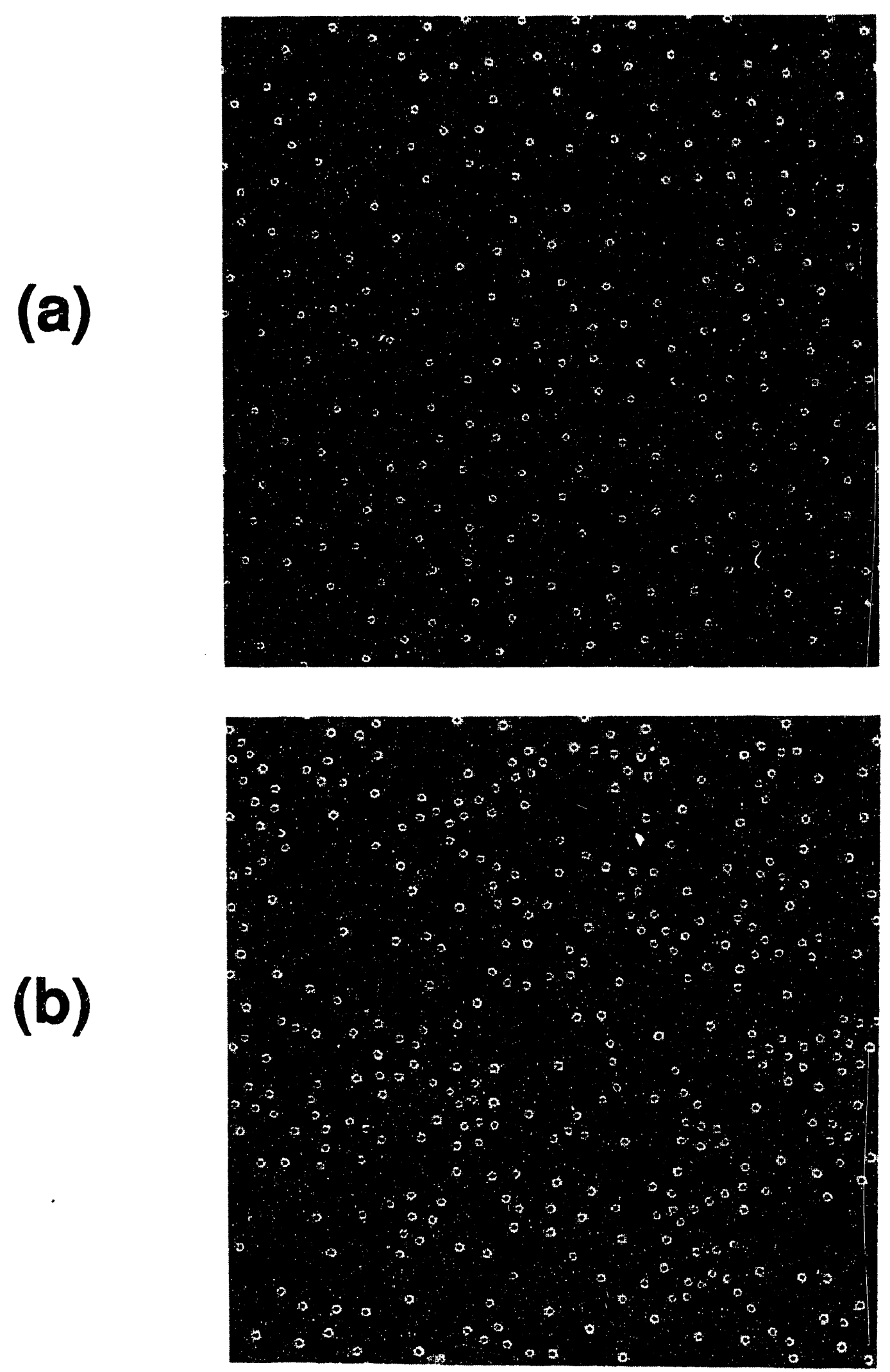

Figure 2.9: Amplitude for (a) $\alpha=0$, (b) $\alpha=0.2$. $(\beta=-1$.) 
(a)
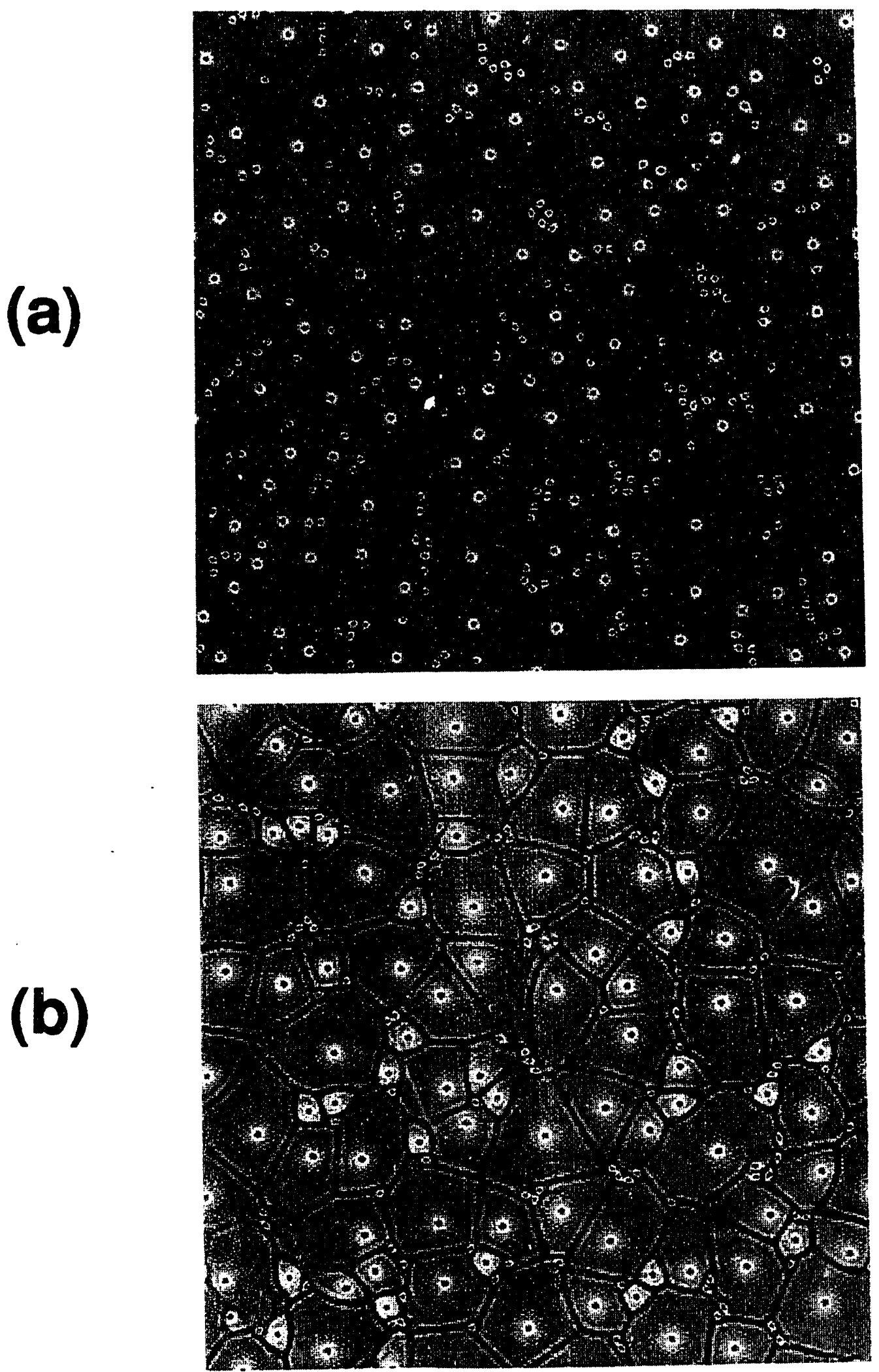

Figure 2.10: Amplitude for (a) $\alpha=0.4$, (b) $\alpha=0.6$. $(\beta=-1$.) 


\subsubsection{Wavelength charts}

It is possible to define a wavelength for the spiral waves in the region where the spiral arms are well-approximated by plane waves. A unique wavelength, a function only of $\alpha$ and $\beta$, is selected by the frozen spiral-wave solutions. Examples of this phenomenon, and data that will be useful in Chapter 4, are presented in Figs. 2.112.13. In each of these sets, $\beta$ is fixed and $\alpha$ is varied

A measurement of this wavelength, for a particular $(\alpha, \beta)$, requires only the existence of a spiral wave. Sufficient time must elapse before a spiral achieves a substantial size (at least a few wavelengths in radius). Depending on the average inter-vortex separation (from the vortex density), large spiral structures may be suppressed, making the wavelength measurement impractical. For an accurate reckoning, some care is taken in the preparation of vortex states. A fast "quench" from an $\alpha$ value corresponding to a low-density vortex state to our particular $\alpha$ of interest (with $\beta$ fixed), will eventually result in a low density of well-formed spirals, all having the sought-after wavelength. Listed in Table 2.1 are the corresponding wavelengths for each of the phase portraits in Figs. 2.11, 2.12, and 2.13, respectively.

\subsection{Turbulent States}

In sharp contrast to the frozen vortex states detailed in Section 2.2, there exist turbulent vortex states characterized by moving vortices and vortex-antivortex pair creation and annihilation. Various methods have been used to analyze this vortex turbulence $[13,22]$.

From the statistical point of view, a suprisingly simple picture has emerged [27]: 


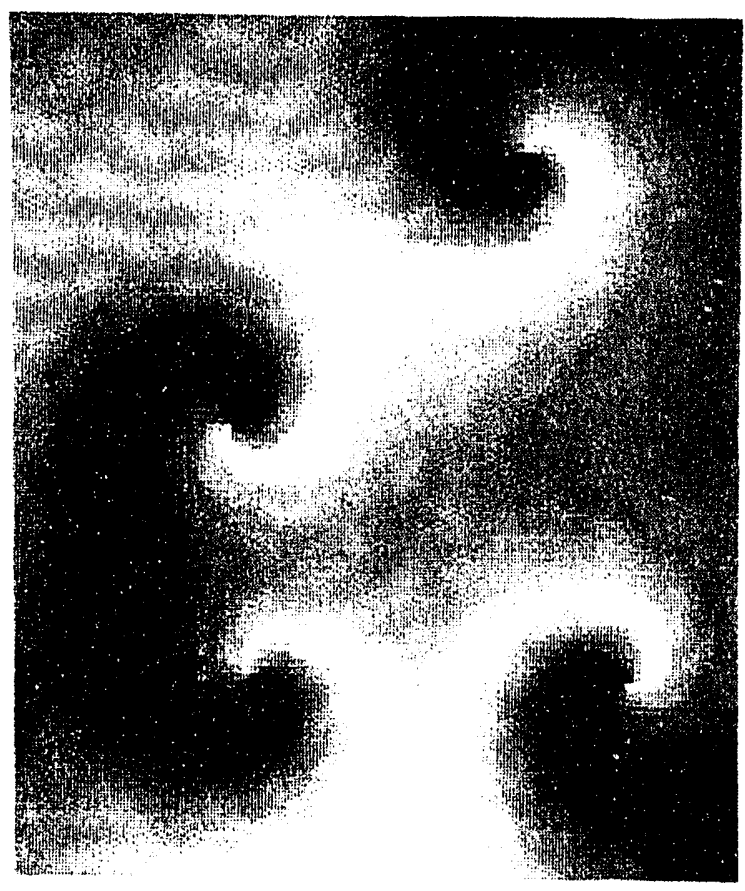

(a)

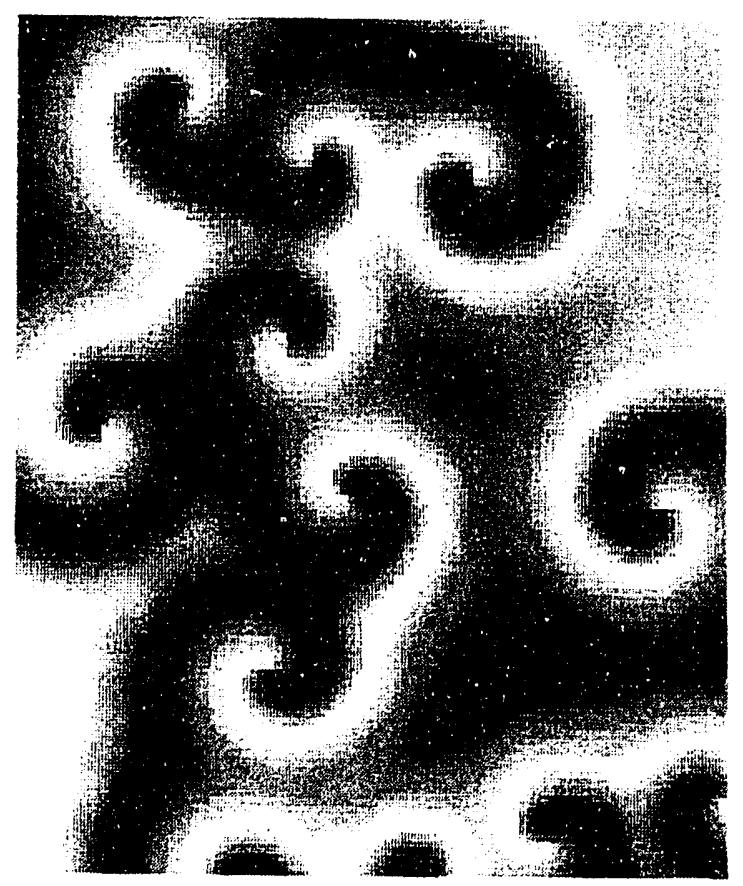

(c)

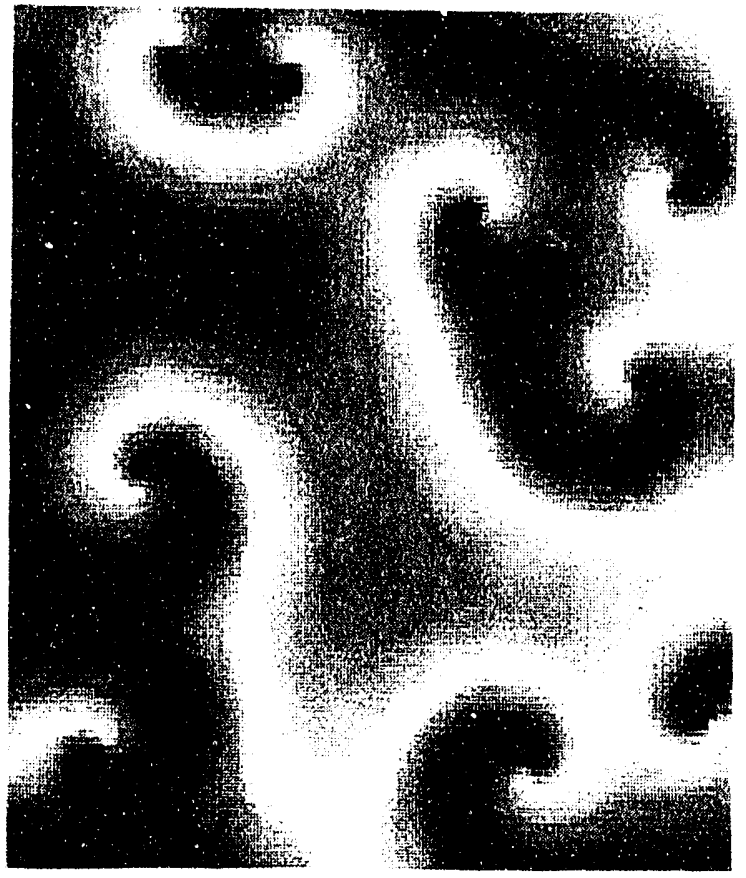

(b)

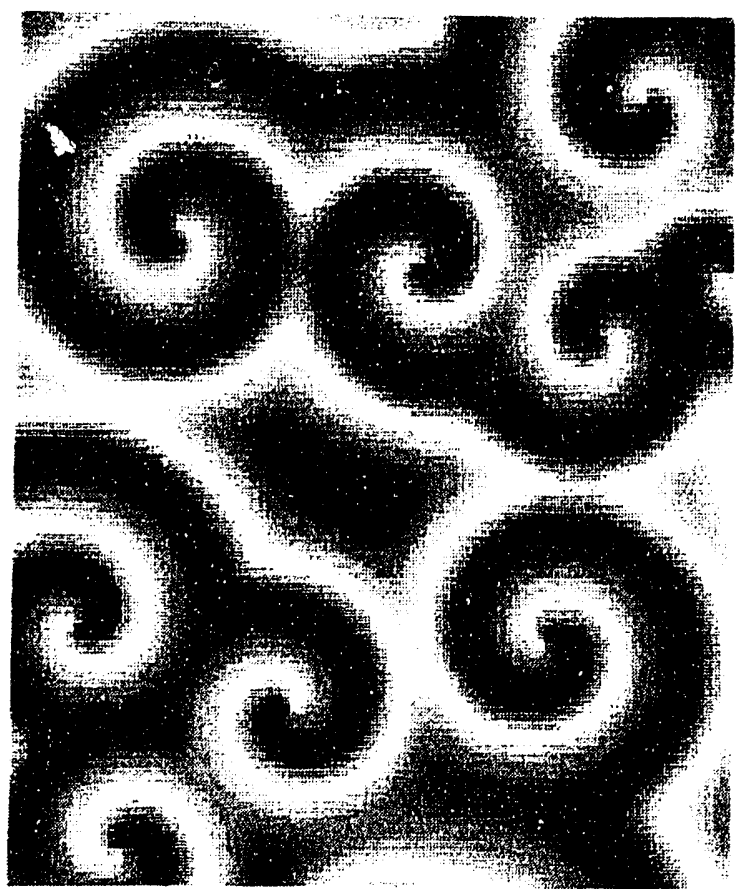

(d)

Figure 2.11: Phase portraits of spiral waves for $\beta=-0.25$ and $\alpha$ values of (a) 0.4 , (b) 0.6 , (c) 0.8 , (d) 1 . Shown are $100 \times 84$ regions of $256 \times 256$ lattices. 


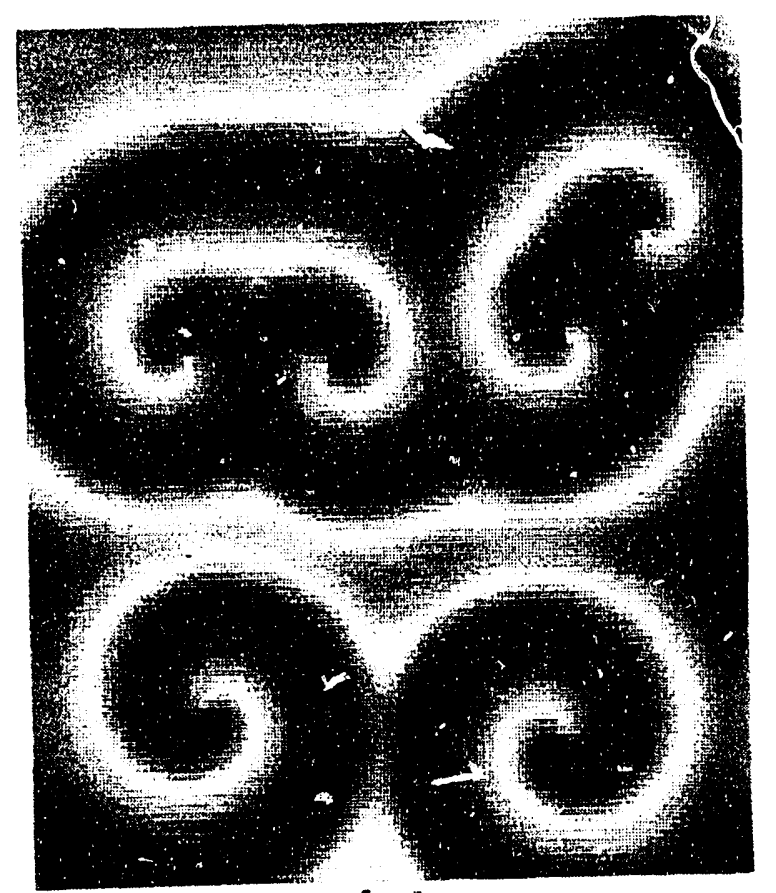

(a)

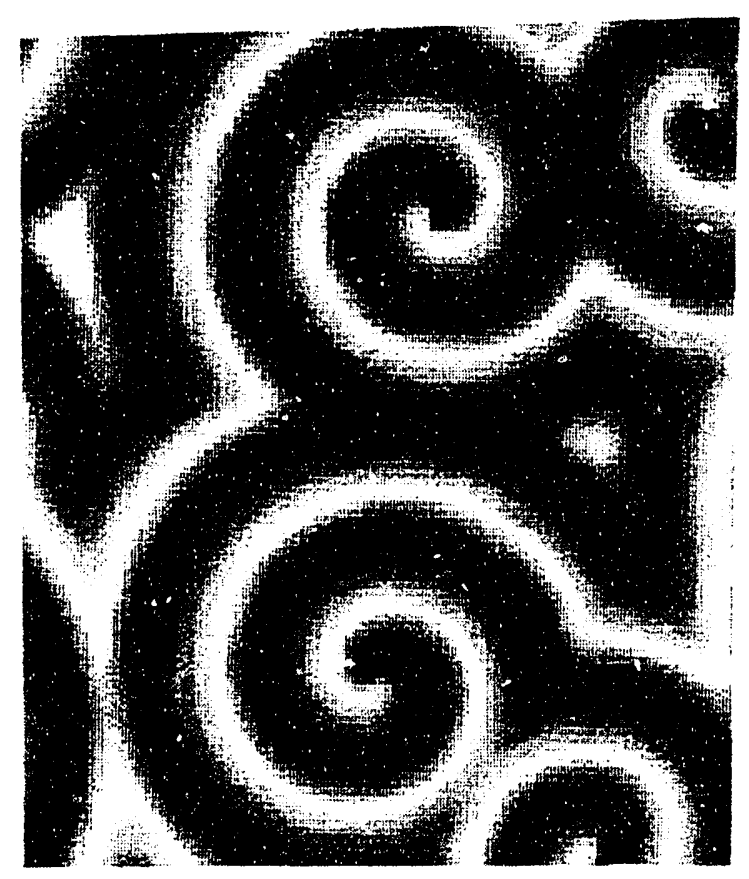

(c)

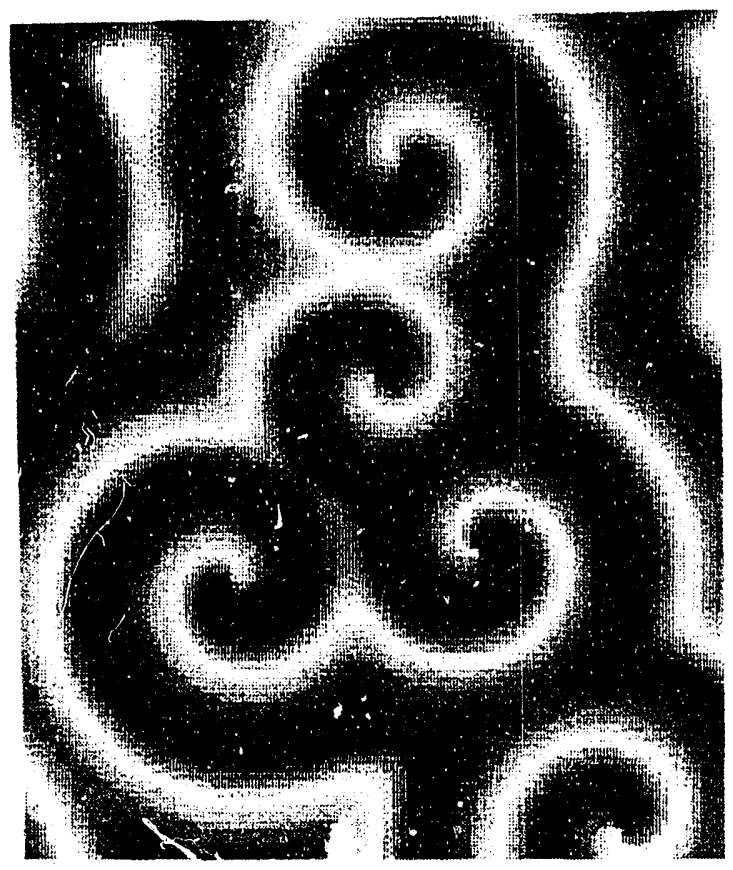

(b)

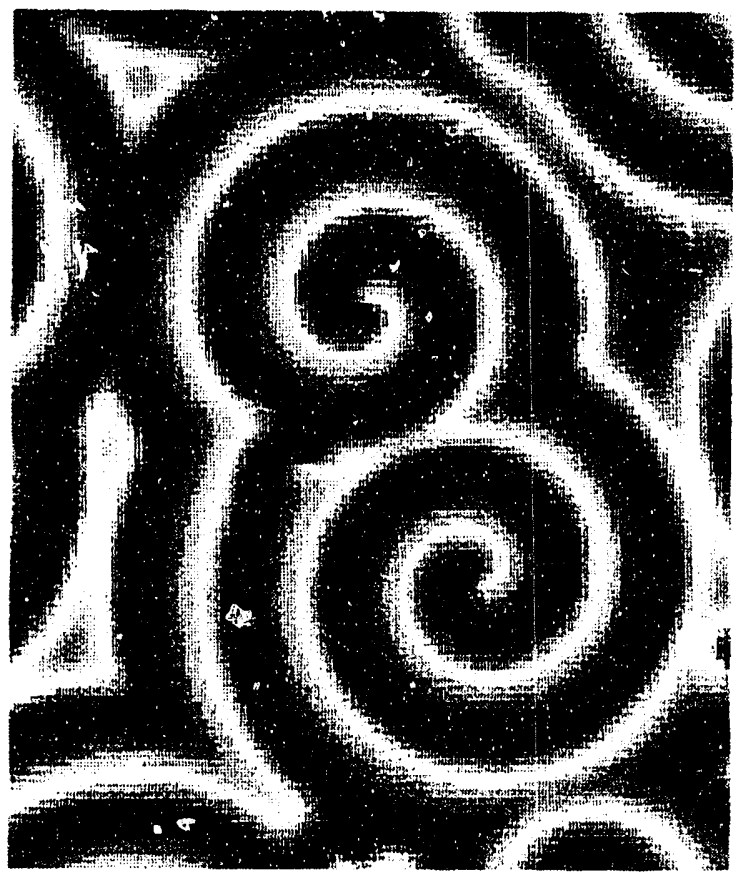

(d)

Figure 2.12: Phase portraits of spiral waves for $\beta=-0.75$ and $\alpha$ values of (a) 0.4, (b) 0.5, (c) 0.6, (d) 0.8 . Shown are $100 \times 84$ regions of $256 \times 256$ lattices. 


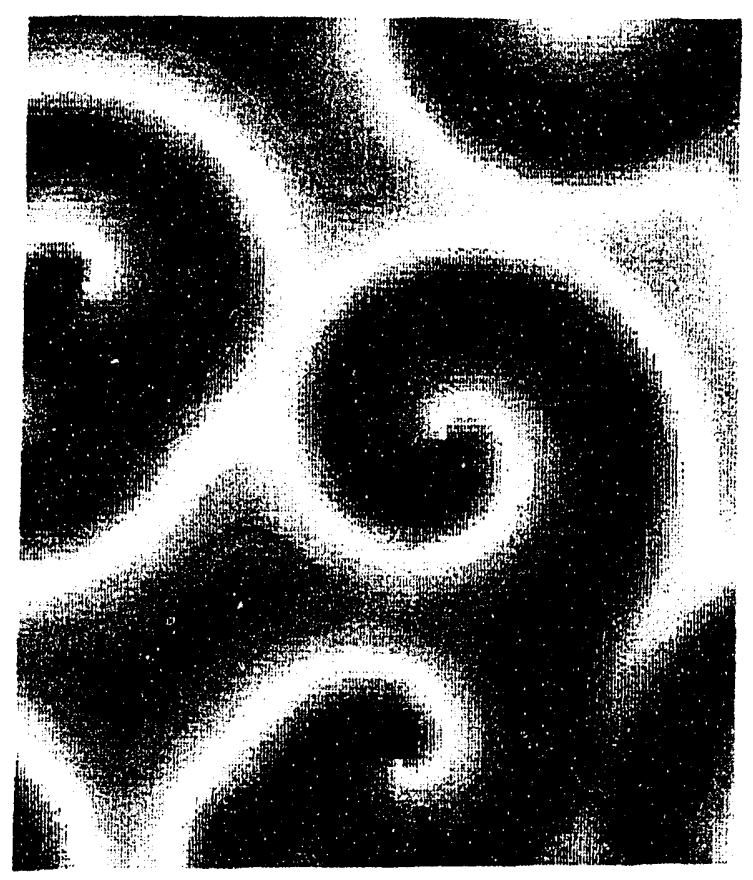

(a)

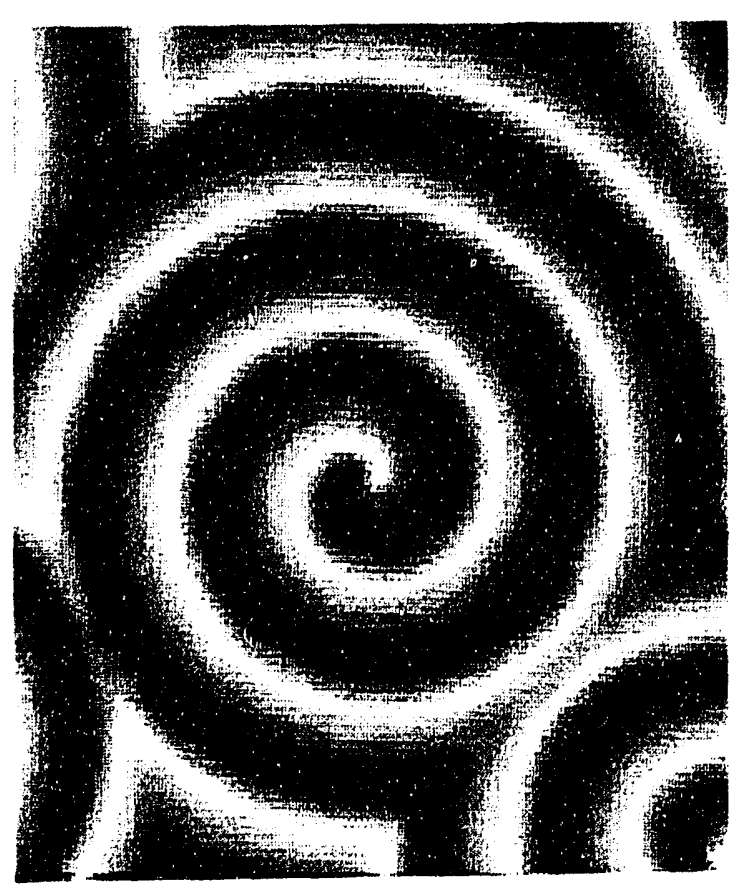

(c)

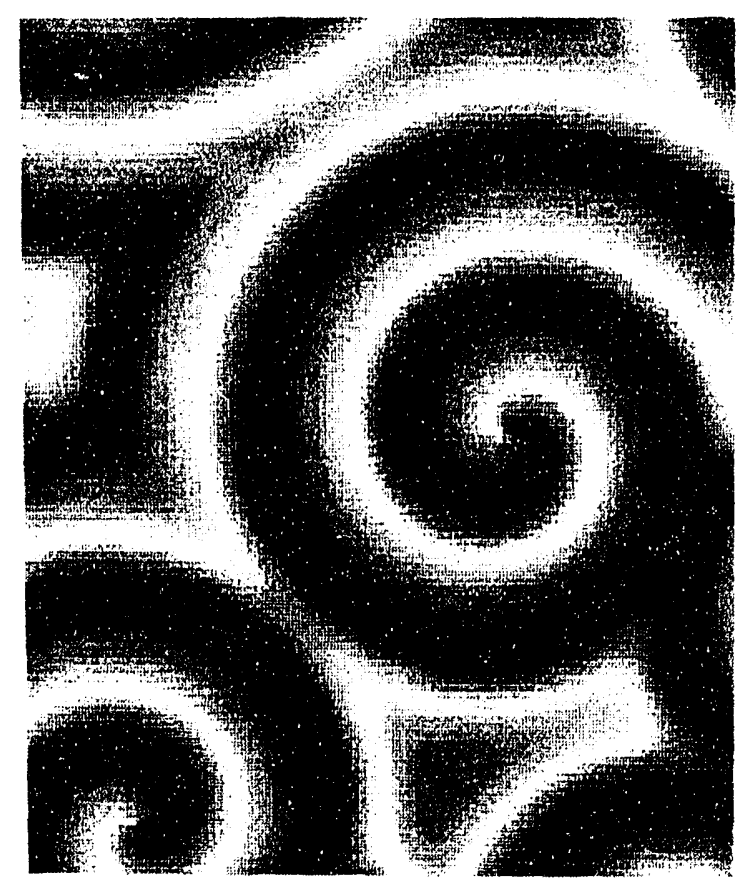

(b)

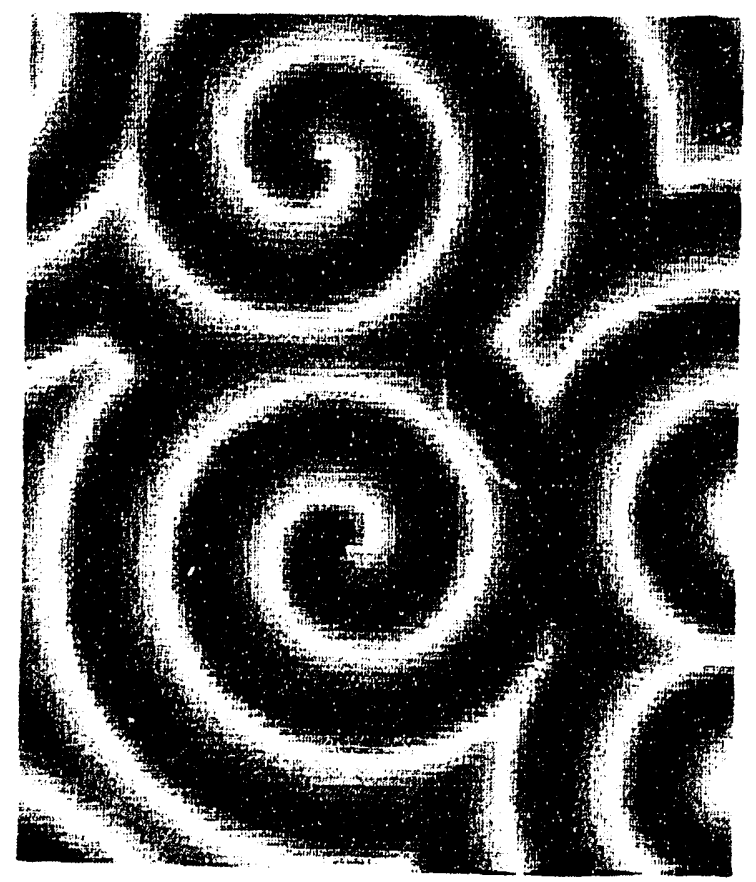

(d)

Figure 2.13: Phase portraits of spiral waves for $\beta=-1.25$ and $\alpha$ values of (a) 0 , (b) 0.2, (c) 0.4 , (d) 0.6 . Shown are $100 \times 84$ regions of $256 \times 256$ lattices. 


\begin{tabular}{|c|c|c|}
\hline \multicolumn{2}{|c|}{$(\alpha, \beta)$} & wavelength \\
\hline 0.4 & -0.25 & $61 \pm 10$ \\
0.6 & -0.25 & 23.9 \\
0.8 & -0.25 & 16.0 \\
1.0 & -0.25 & 14.4 \\
\hline
\end{tabular}

\begin{tabular}{|c|c|c|}
\hline \multicolumn{2}{|c|}{$(\alpha, \beta)$} & wavelength \\
\hline 0.4 & -0.75 & 17.55 \\
0.5 & -0.75 & 16.1 \\
0.6 & -0.75 & 15.05 \\
0.8 & -0.75 & 13.2 \\
\hline
\end{tabular}

\begin{tabular}{|c|c|c|}
\hline \multicolumn{2}{|c|}{$(\alpha, \beta)$} & wavelength \\
\hline 0.0 & -1.25 & 22.2 \\
0.2 & -1.25 & 18.6 \\
0.4 & -1.25 & 16.2 \\
0.6 & -1.25 & 13.75 \\
\hline
\end{tabular}

Table 2.1: Spiral wavelengths for various $\alpha ; \beta=-0.25,-0.75$, and -1.25 . 
Vortices (and antivortices) are uniformly distributed in space, and the average separation between neighboring defects is of order the correlation length of the field $A$. The latter fact is ascertained from measurements of the time-averaged correlation function $\left\langle\operatorname{Re}\left[A(\mathbf{r}) A^{*}(\mathbf{0})\right]\right\rangle$, which has been found to decrease exponentially with $|\mathbf{r}|$ $[27]$.

One striking characteristic of the turbulent states is their relatively high density. That there should be a "steady-state" density, can be seen as follows: Since the field correlation is as large as the vortex separation, one can assume a constant rate $c_{+}$of pair creation "far" from any extant vortices. Furthermore, since vortices annihilate by pairs, the rate of annihilation can be taken proportional to the vortex density squared, namely $c_{-} \rho^{2}$. The steady-state density that balances these two terms is then $\rho=\sqrt{c_{+} / c_{-}}$. (See Section 3.2 for more about this simple creation-annihilation model.)

For the turbulent states, we also present a visual catalog of sorts. We fix $\alpha$ at a relatively large value $(\alpha=2)$, and vary $\beta$ from 0 to -1.5 . The resulting amplitude portraits, made at a time $t=2000$, are presented in Figs. 2.14a-e.

\subsection{Transition Line}

Our numerical work has shown that, in the limit of long times $\left(\approx 10^{5}\right)$, the system either remains turbulent, or settles into a low-density state of frozen, randomlydistributed vortices, separated by well-defined domain walls [18]. The transition line $\alpha=\alpha_{0}(\beta)$ between these two phases can be found through the following procedure: Fix $\beta$, and choose a small $\alpha$ to ensure that the system will enter a frozen vortex state; increment $\alpha$ slightly with each time step. At some $\alpha$, the vortex density will 


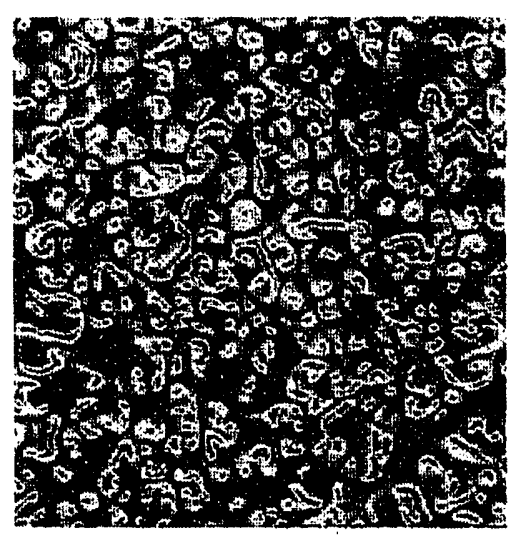

\section{(a)}

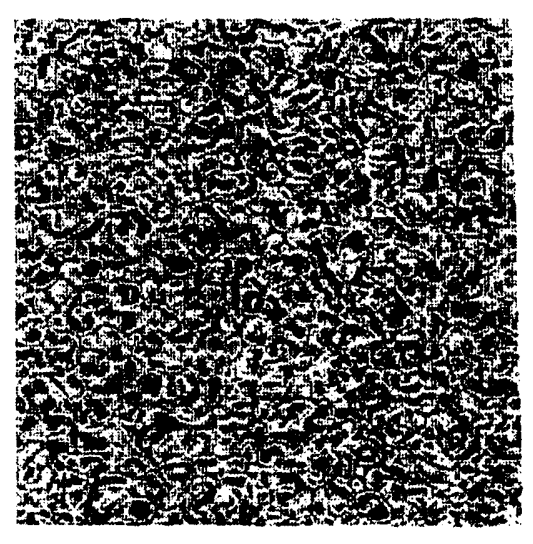

(c)

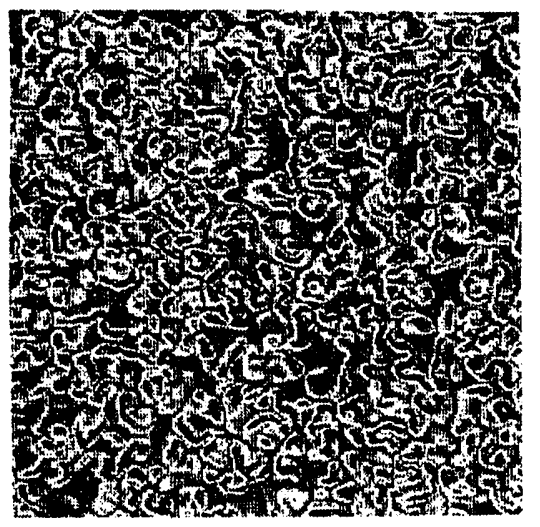

(b)

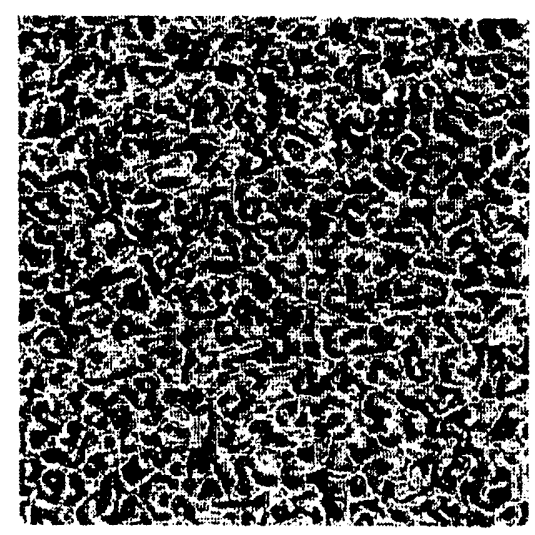

(d)

Figure 2.14: Amplitude portrait for $\alpha=2$ and $\beta=$ values of (a) 0 , (b) -0.5, (c) $-1,(d)-1.5$. 


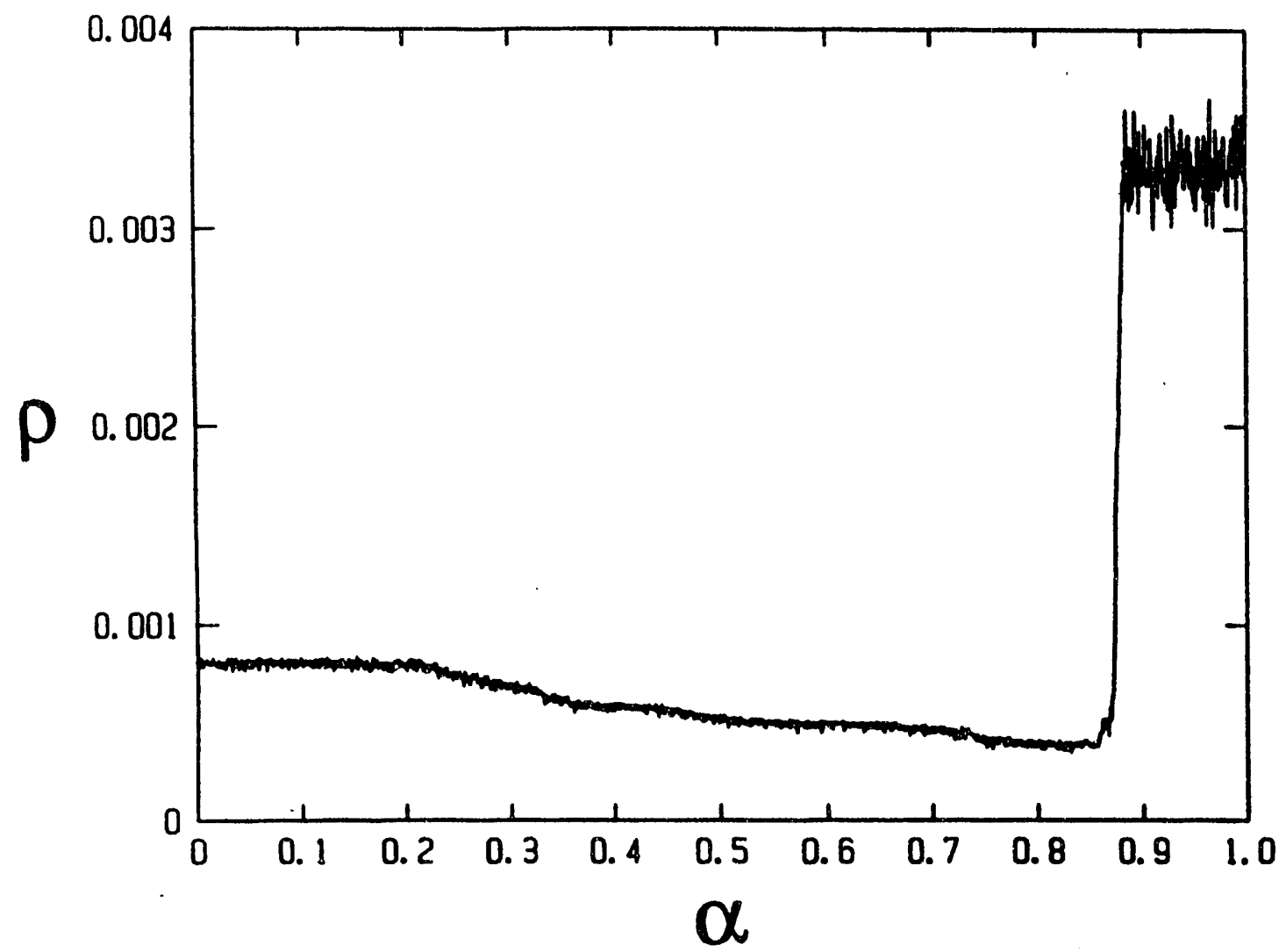

Figure 2.15: Vortex density as a function of $\alpha$, for $\beta=-1$. Density obtained by slowly increasing $\alpha$ at the rate $\dot{\alpha}=5 \cdot 10^{-6}$.

increase abruptly by an order of magnitude (cf. Fig. 2.15). The transition $\alpha$ can be read off from the position of the density jump. For example, averages over a few runs like that of Fig. 2.15 (where $\beta=-1$ and $\dot{\alpha}=5 \cdot 10^{-6}$ ) put the transition at $\alpha_{0}=0.855 \pm 0.003$. By systematically measuring $\alpha_{0}$ for a sequence of decreasing $\dot{\alpha}$, we can extrapolate to the limit of zero rate. For the above example, we found the asymptotic value $\alpha_{0}=0.8458$.

Performing the same analysis for a range of $\beta$ gives us the transition line to 


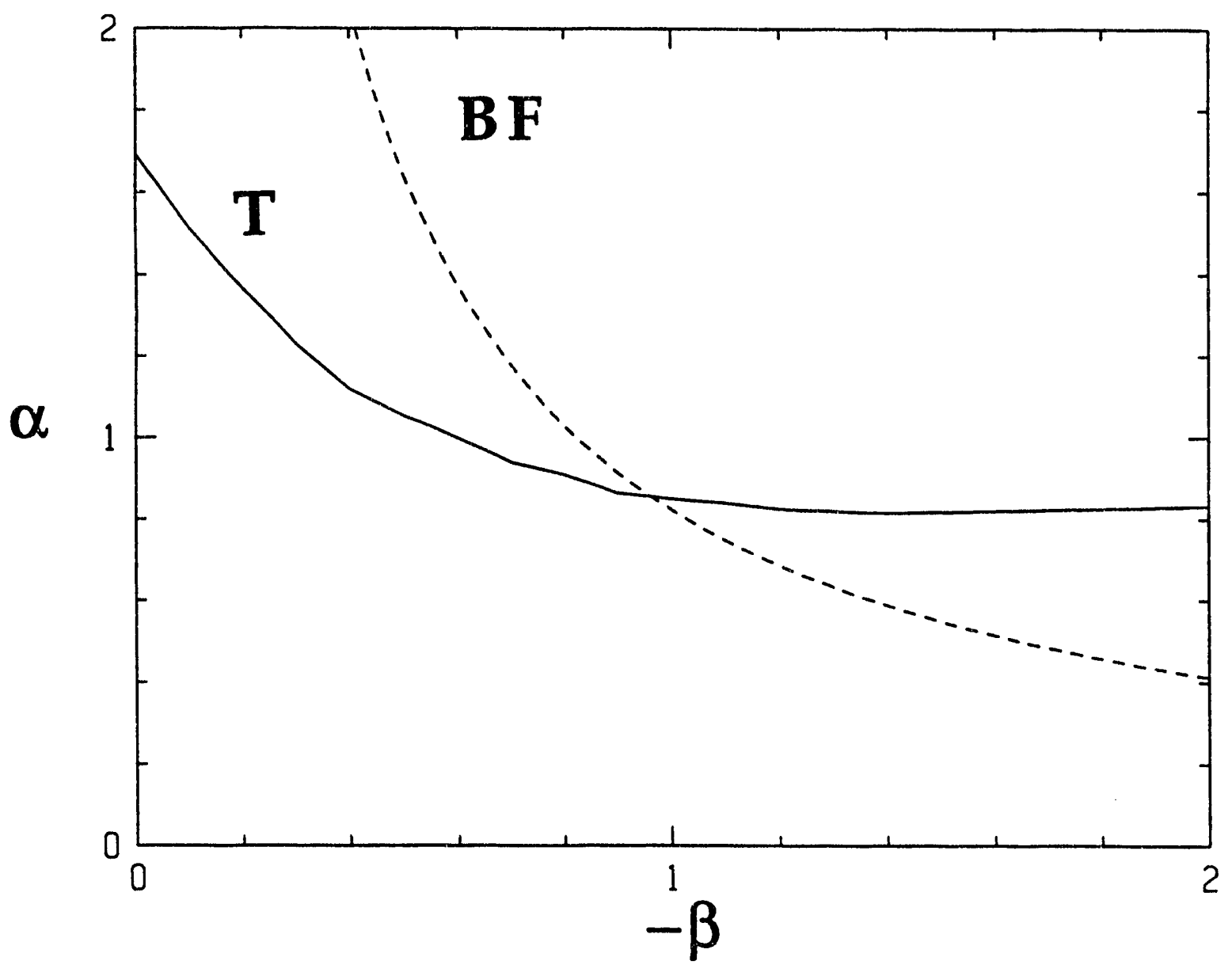

Figure 2.16: Phase diagram for $0 \leq \alpha \leq 2$, and $-2 \leq \beta \leq 0$. (T) Transition line $\alpha_{0}(\beta)$ to vortex turbulence (solid). (BF) Benjamin-Feir line (dashed). 
vortex turbulence $\alpha_{0}(\beta)$. We compare this line with the Benjamin-Feir line in Fig. 2.16. It is remarkable that the transition to turbulence can take place both below and above the Benjamin-Feir line. This directly contradicts the prediction that turbulence lies entirely within the Benjamin-Feir unstable region. Within the last year, a group in Germany has performed simulations based on pseudo-spectral codes and has confirmed our turbulence transition line [28]. 


\section{Chapter 3}

\section{Vortex Density and Decay}

\subsection{Density Traces}

There is a superficial similarity between the dynamics of the CGL equation with random initial conditions, and the relaxational, ordering behavior of deeply-quenched thermodynamic systems. For the latter case, the theory of phase-ordering processes [29] predicts the development of patterns characterized by a dynamical length having a power-law scaling in time. The existence of frozen vortex states (see Section $2.2)$, and therefore of frozen length scales, would seem to preclude this type of scaling law. However, if the system decays smoothly to zero density, then there is no obstacle to applying the phase-ordering ideas. This possibility is explored in Section 3.5.

In the absence of a theory of the dynamical phases in the CGL systenı, w' find that the plot of vortex number (or density) versus time provides a tool for directly tracing the different dynamical regimes. As an example representing the variety of dynamical changes, consider a $512 \times 512$ lattice with the parameter values 


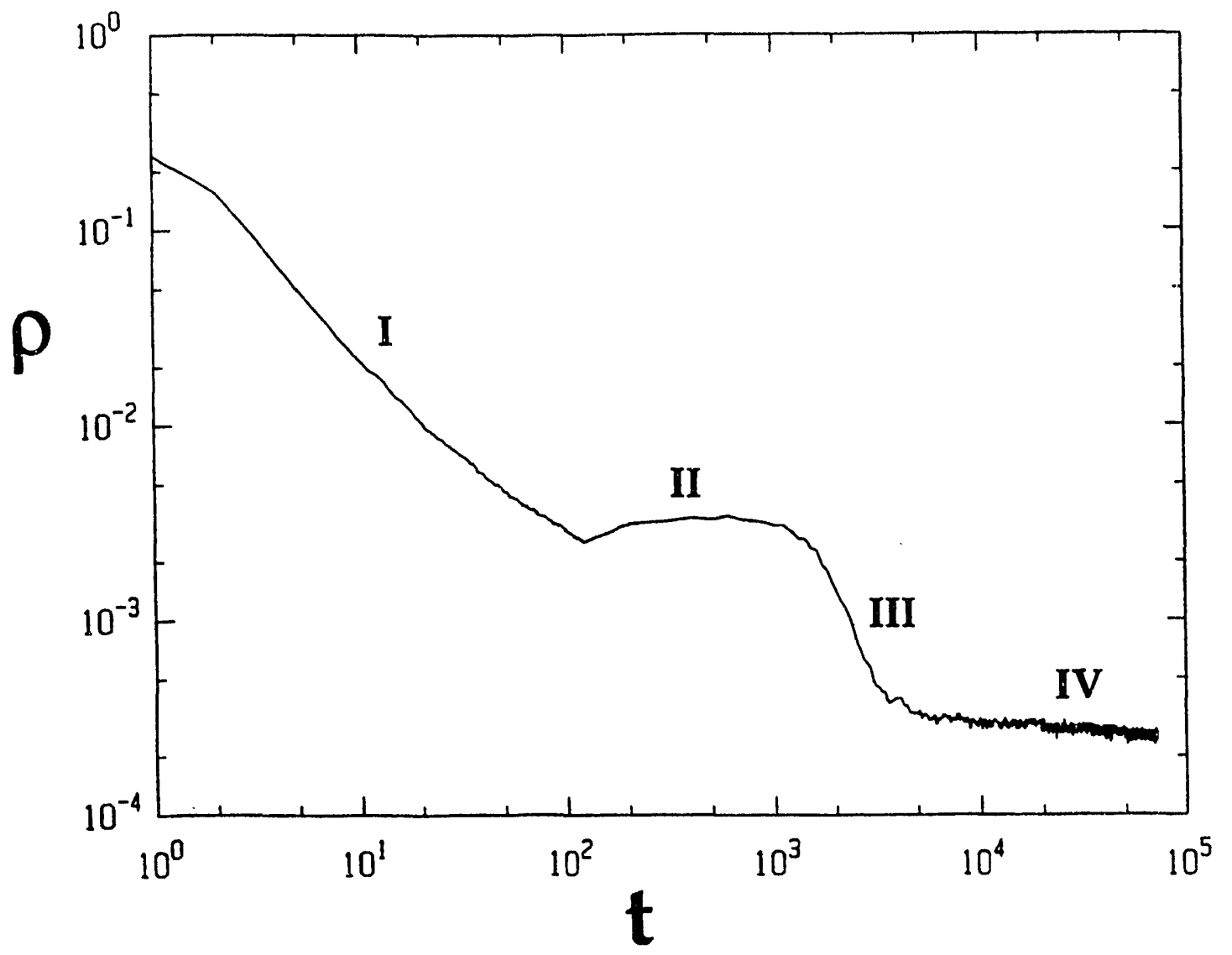

Figure 3.1: Decay of vortex density in time, from random initial conditions, for $\alpha=0.75, \beta=-1$. Four dynamical regimes (I)-(IV) are indicated.

$\alpha=0.75, \beta=-1$ (Fig. 3.1). Four distinct dynamical behaviors can be identified: (I) initial decay, (II) transient steady state, (III) decay to frozen state, (IV) frozen vortex state.

More generally, let us take a vertical slice through the $\alpha-\beta$ phase diagram (choosing here $\beta=-1$ ), and examine the density plots for a siccession of different $\alpha$ values. Fig. 3.2 is the result of this sweep, which examined a range of $\alpha$ from 0 to 2 , out to a time $t=10^{5}$ (plotted here to $t=72000$ so that the vertical scale could be read). Only the density traces labeled $c, d$ and $e$ of Fig. 3.2 can be placed in the same category as the trace in Fig. 3.1. These are distinguished by a local density minimum (between regimes I and II), a plateau-like transient (II), and a 


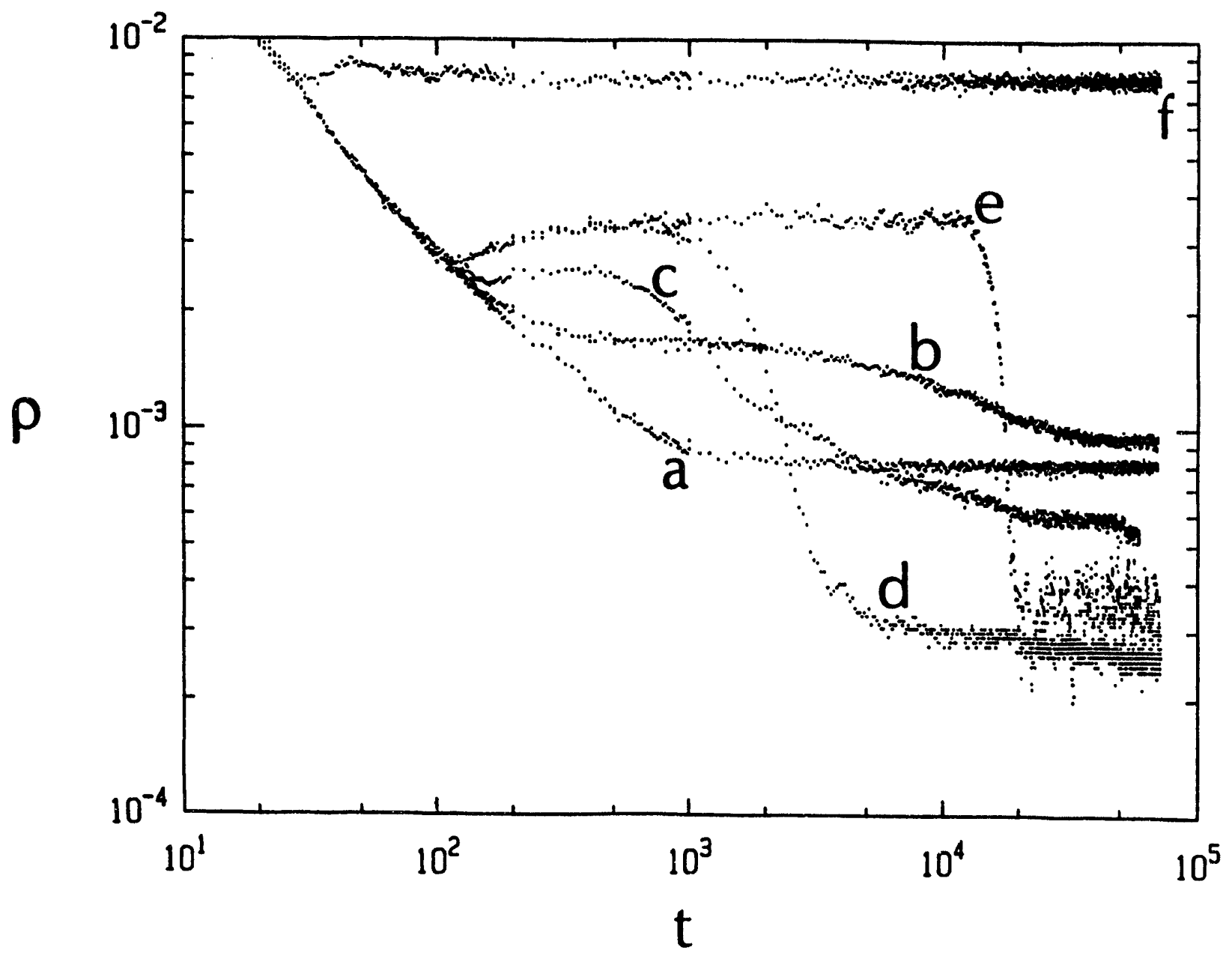

Figure 3.2: Vortex density $\rho(t)$ obtained starting from a random initial state for $\beta=-1$. The $\alpha$ values correspond to (a) 0 , (b) 0.25 , (c) 0.6 , (d) 0.75 , (e) 0.8 , and (f) 2 . 
relatively rapid drop in density (III). (Trace also displays a density minimum, bul followed by a presumably eternal turbulent state. This agrees with our previous placement of the turbulence transition line, i.e. case $f$ is far above the transition to turbulence.)

About the traces labeled by $a$ and $b$, we shall say very little in this work. Very slow decay to the frozen state (like class $b$ ) is briefly discussed in Section 3.3.

\subsection{Initial Decay}

The region of initially rapid decay (from times of order one, to a time $T_{s}$ of order 100 near the transition to turbulence) is well fit by a line of slope -1 . The fit is shown in Fig. 3.3. The same initial behavior is seen over the entire phase diagram, for (asymptotically) frozen and turbulent phases, independent of $\alpha$ and $\beta$. We can think of this rapid decay as stemming from the extremely high density of defects generated in the random initial conditions. The consequent decay in vortex density can be understood from a simple picture of vortex-antivortex annihilation and creation. The basic assumption is mean-field: the rate of annihilation is proportional to the density squared, since the density can only decrease through vortex-antivortex encounters. In other words, $\dot{\rho} \propto-\rho^{2}$, and this leads to the power-law decay $\rho \propto 1 / t$, as observed.

Moreover, an additional contribution comes from the creation of vortexantivortex pairs. We assume that, at these high initial densities, the pair creation rate is roughly a constant, $c_{+}$. The time rate-of-change of the density is then

$$
\dot{\rho}=c_{+}-c_{-} \rho^{2}
$$




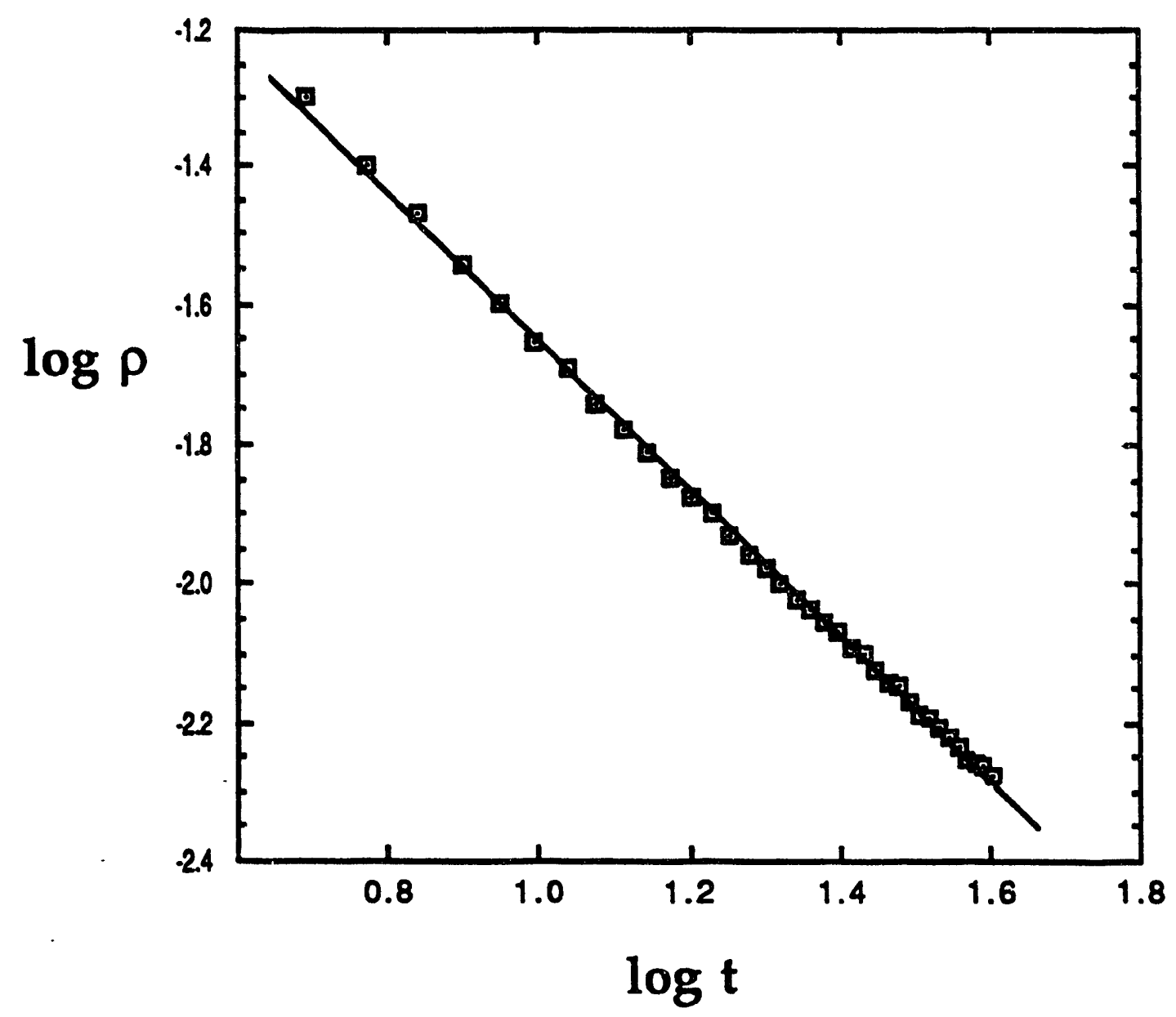

Figure 3.3: $\log -\log$ plot of density versus time, for $\alpha=0.8, \beta=-1$ and $512 \times 512$ system. Best-fit slope (shown) is $-1.06 \pm 0.04$. 
Recasting this equation in term of a rescaled density, $\rho=\sqrt{c_{+} / c_{-}} \rho^{\prime}$, gives

$$
\dot{\rho}^{\prime}=\sqrt{c_{+} c_{-}}\left(1-\rho^{2}\right) .
$$

Note that the derivative of $\operatorname{coth}(t)$ is simply $1-\operatorname{coth}^{2}(t)\left(\right.$ using $\cosh ^{2}(t)-\sinh ^{2}(t)=$ 1), and so the derivative of $\operatorname{coth}(k t)$ is $k-k \operatorname{coth}^{2}(k t)$. The solution to (3.1) is then easily read off from the above:

$$
\rho=\sqrt{c_{+} / c_{-}} \operatorname{coth}\left(\sqrt{c_{+} c_{-}} t\right)
$$

For small $t,(3.3)$ reduces to $\rho=\left(c_{-} t\right)^{-1}$, as argued above. On the other hand, for large $t,(3.3)$ approaches the constant $\sqrt{c_{+} / c_{-}}$. A more detailed version of this argument has been published [27].

With the initial decay curve showing no dependence on $\alpha$ and $\beta$, the fit in Fig. 3.3 provides an estimate of $c_{-} \simeq 4$. For an estimate of $c_{+}$, we need the initial decay to bottom out to a turbulent steady state, which we could take to be transient or non-transient. However, in either case, the density goes through a local minimum at a time $T_{s}$ before flattening out - behavior not allowed by our naive solution (3.3). Nevertheless, we can argue that the steady state density still corresponds to the constant $\sqrt{c_{+} / c_{-}}$found above. For example, in the case $\alpha=0.8, \beta=-1$ (trace $e$ in Fig. 3.2), this identification gives $c_{+} \simeq 4.5 \cdot 10^{-5} ;$ in the case $\alpha=2.0, \beta=-1$, we get $c_{+} \simeq 2.5 \cdot 10^{-4}$.

In this view, the dip in density would then correspond to the system undershooting and then returning to the steady state, as if the system, despite the underlying first order $(\dot{A})$ dynamics, had a second-order term enabling it to slosh back and forth in a potential well. 


\subsection{Slow decay to Frozen State}

The slow decay to a frozen state, for example trace $b$ of Fig. 3.2 , stands somewhat outside the domain of immediate concern in this thesis. Inasmuch as the features of the slow decry remain poorly understood, this case would merit a full chapter in a more complete work. However, at the very least, it is instructive to see the amplitude plots at different times during this slow decay. Figs. 3.4-3.6 are the amplitude fields, taken from a $1024 \times 1024$ system, for times $t=100,400,10^{4}$, respectively.

Some interesting information can be gleaned from these amplitude plots. By time 400, a high-density state with well-defined domain walls has formed. This state is wearly frozen. Between times 400 and $10^{4}$, the random lattice has decreased in vortex density, and regrouped considerably. A segregation into "large" vortices and "small" vortices has occurred, with the small vortices clumped into separate clusters of size comparable to a single large vortex. We classify this state as frozen, although from time to time a domain wall breaks down, resulting in vortex-antivortex annihilation. The segregation, rearrangernent and subsequent pair annihilation of the small, clump-bound vortices and antivortices are responsible for the very slow decay in overall density.

\subsection{Fast Decay to Frozen State}

\subsubsection{Parabola law: Numerics}

The slow decay featured in the last section disappears for higher a values that are still below the transition to turbulence. These systems possess a transient turbulent 


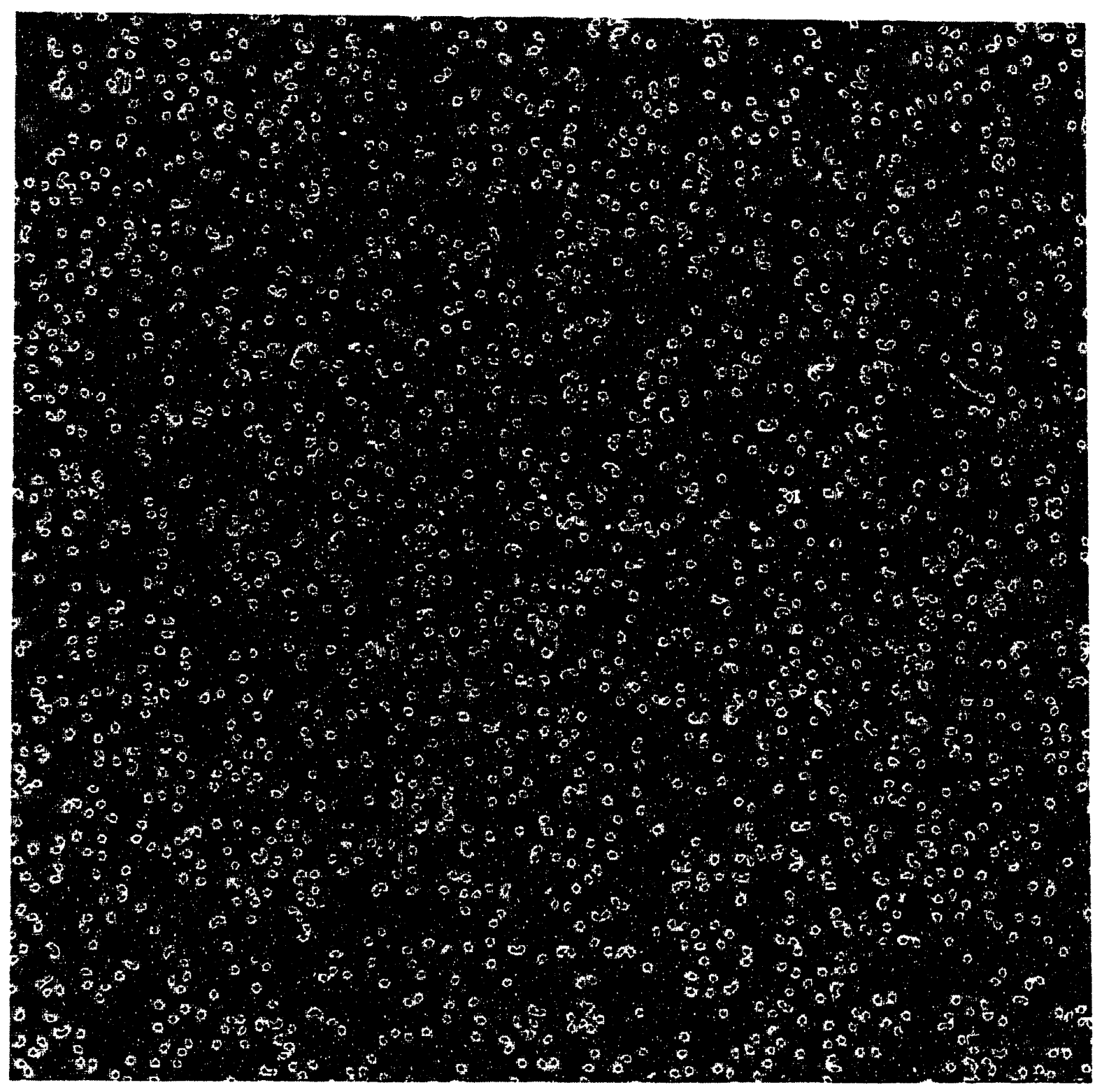

Figure 3.4: Amplitude for $\alpha=0.25, \beta=-1$, at time $t=100.760 \times 760$ region of a $1024 \times 1024$ lattice. 


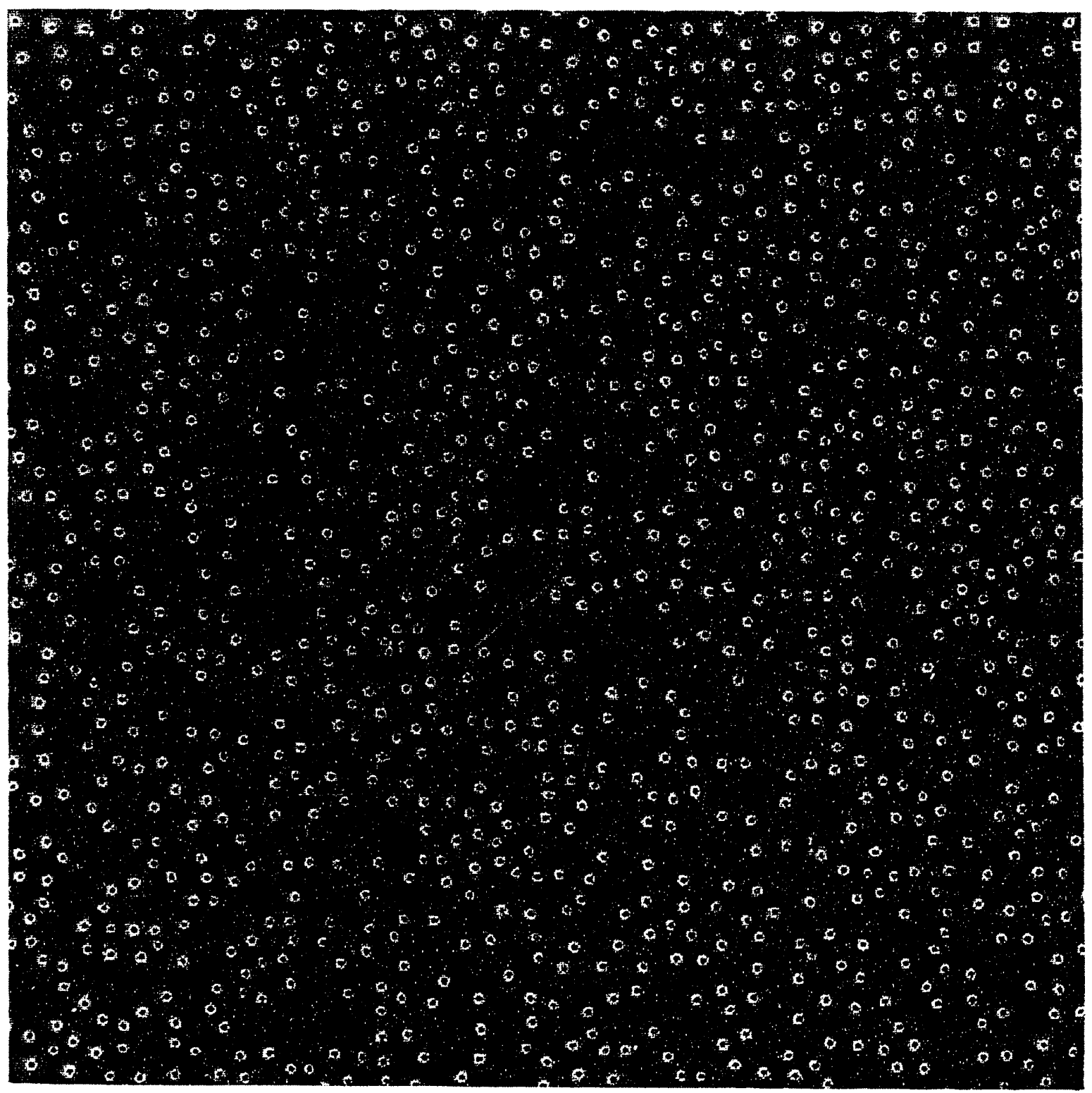

Figure 3.5: Amplitude at $t=400$, otherwise same parameters as in Fig. 3.4. 


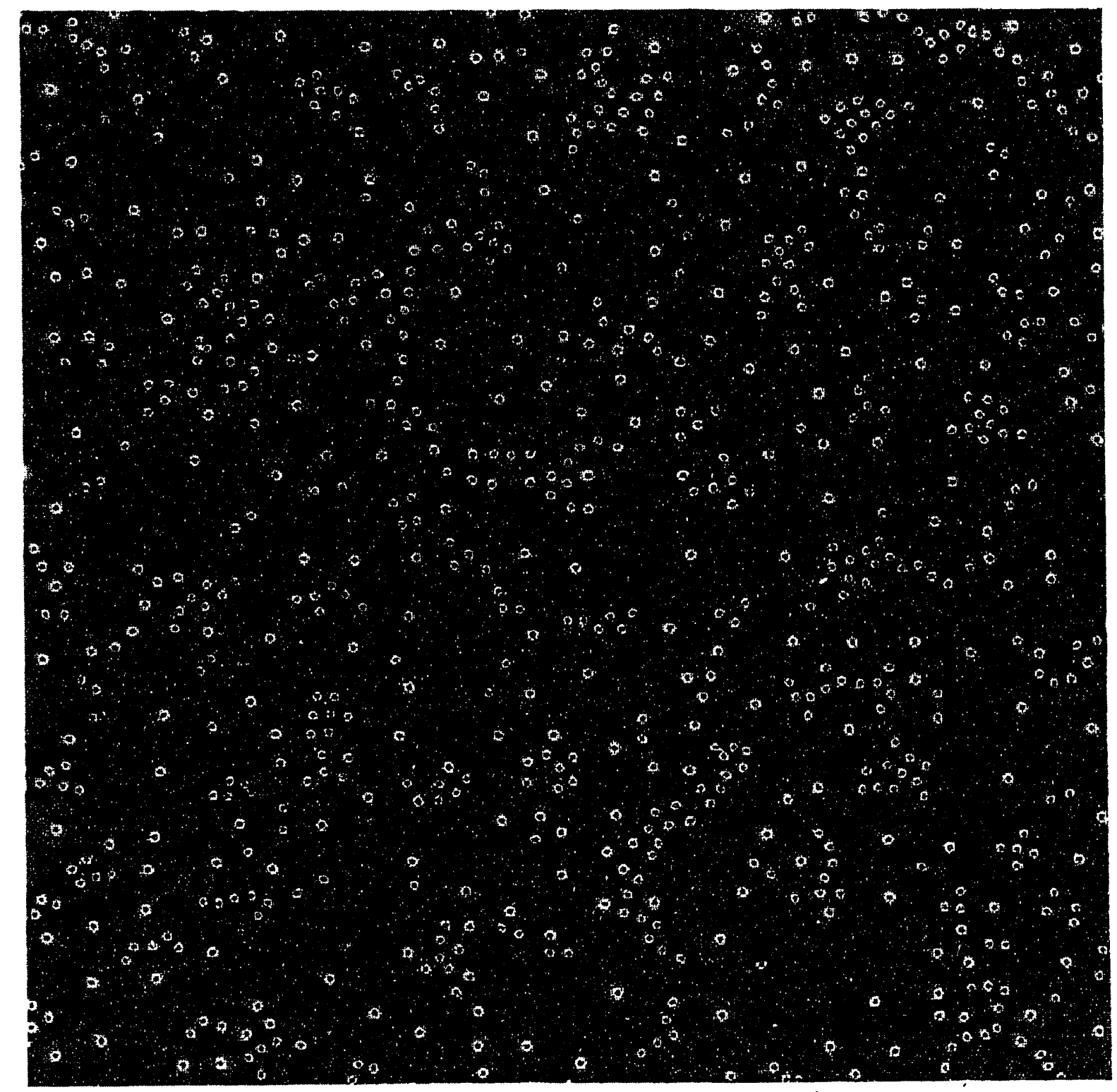

Figure 3.6: Amplitude at $t=10^{4}$, otherwise same parameters as in Fig. 3.4. 
state, and at some point rapidly decay out of this state to a frozen configuration. As we devote all of Chapter 4 to studying the transients (regime II in Fig. 3.1), in this section we content ourselves with considering the decay from the high, steady-state density to the low density of the frozen state (regime III of same figure).

We begin by redisplaying the fast decay data in Fig. 3.2. Cases $d$ ( $\alpha=0.75, \beta=$ $-1)$ and $e(\alpha=0.8, \beta=-1)$ in that plot have been plotted in Fig. 3.7 with linear density and linear time axes. The decay region, for much of its extent, appears to have a parabolic shape. Fig. 3.7 gives the best quadratic fit to each case. Let the quadratic $\rho=a+b t-c t^{2}$ be denoted by $(a, b,-c)$. Then the two curves are $d:\left(0.00345,2.63 \cdot 10^{-7},-6.55 \cdot 10^{-10}\right)$ and $e:\left(-0.00442,1.42 \cdot 10^{-6},-6.35 \cdot 10^{-11}\right)$. Shifting the time origin to the position of the maximum, yields the following two equations:

$$
\begin{aligned}
& \rho(\alpha=0.75)=0.00348-6.55 \cdot 10^{-10} t^{2}, \\
& \rho(\alpha=0.80)=0.00355-6.35 \cdot 10^{-11} t^{2} .
\end{aligned}
$$

\subsubsection{Nucleation}

To understand the parabola laws $(3.4,3.5)$ it helps to view the system as it decays out of the turbulent transient. To this end, we present in Figs. 3.8-3.10 amplitude portraits of a large system, with $\alpha=0.791, \beta=-1$, at an early, intermediate, and final stage of the decay, respectively. The phenomenon we observe in these pictures is nucleation. Vortex "droplets" nucleate out of the turbulent sea, and grow until the entire space is covered by the frozen phase. We therefore think of the transient turbulent state as unstable with respect to localized fluctuations of finite size; in other words, the transient state is metastable. We return to this idea in Chapter 4 , 


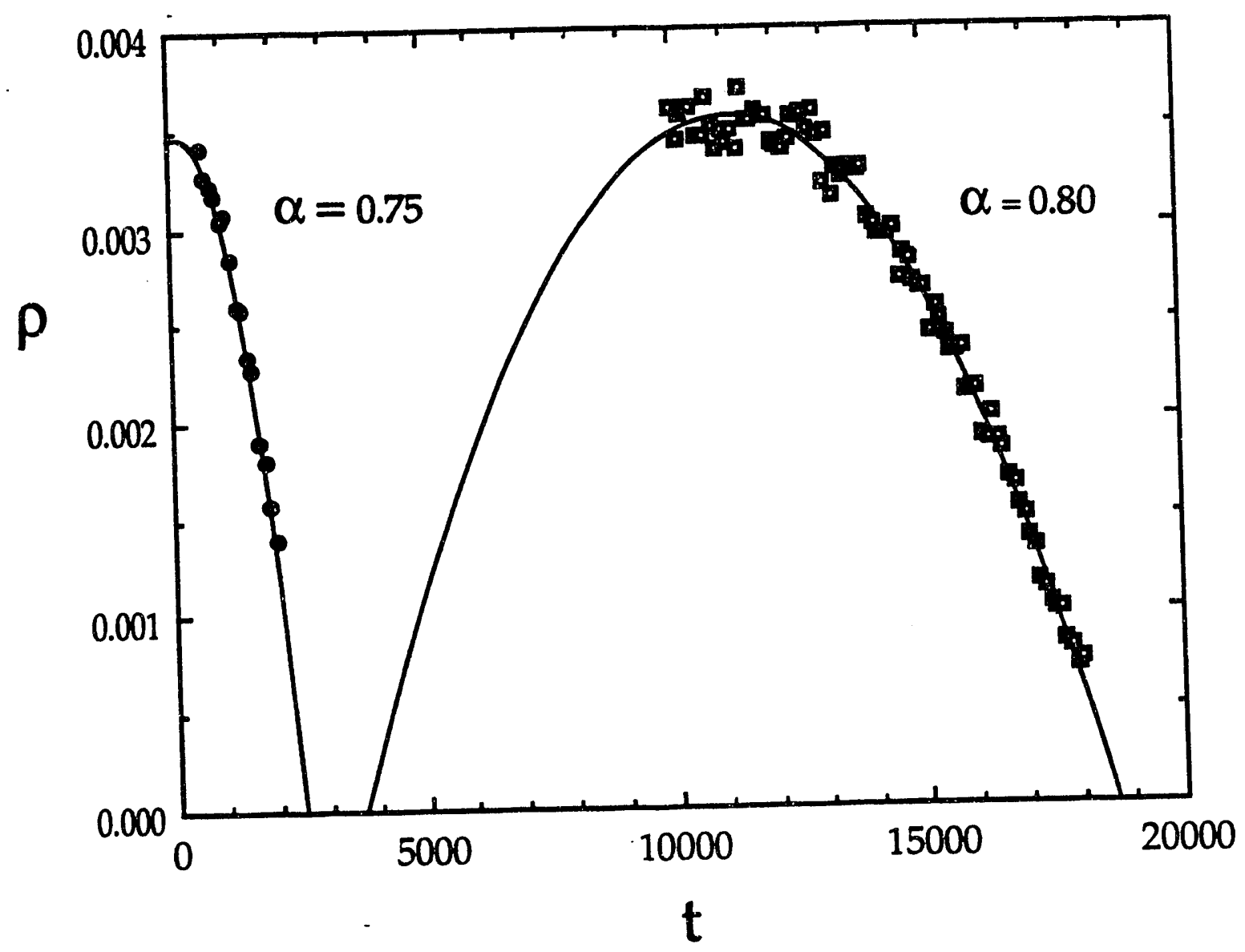

Figure 3.7: Density versus time for fast decay to frozen vortex state. Curves are best fits of the form $\rho=a+b t-c t^{2}$. Left curve: $\alpha=0.75, \beta=-1$. Right curve: $\alpha=0.8, \beta=-1$. 


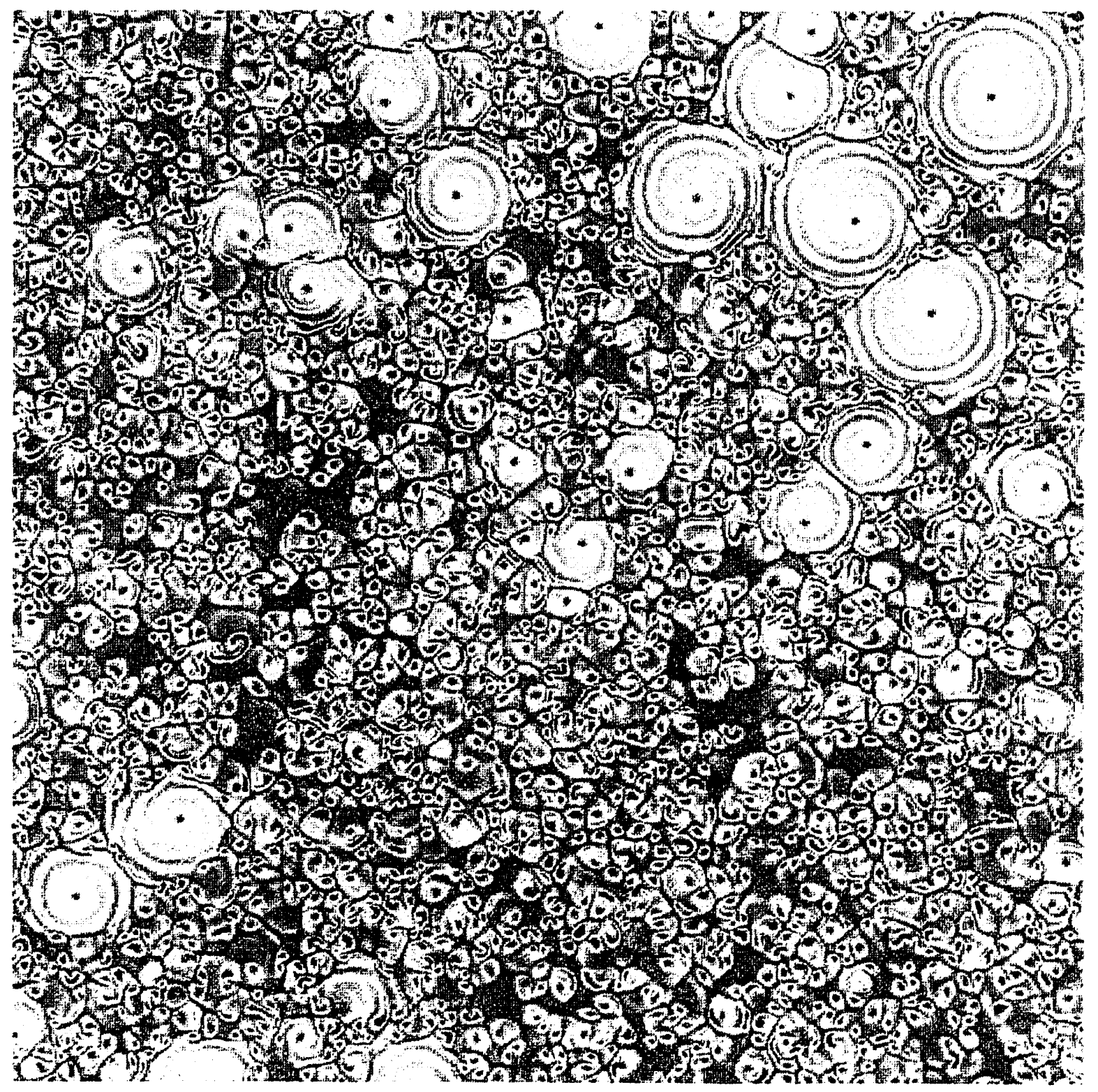

Figure 3.8: Beginning of nucleation, for $\alpha=0.791, \beta=-1$. Shown is an $760 \times 760$ region of a $1024 \times 1024$ lattice. 


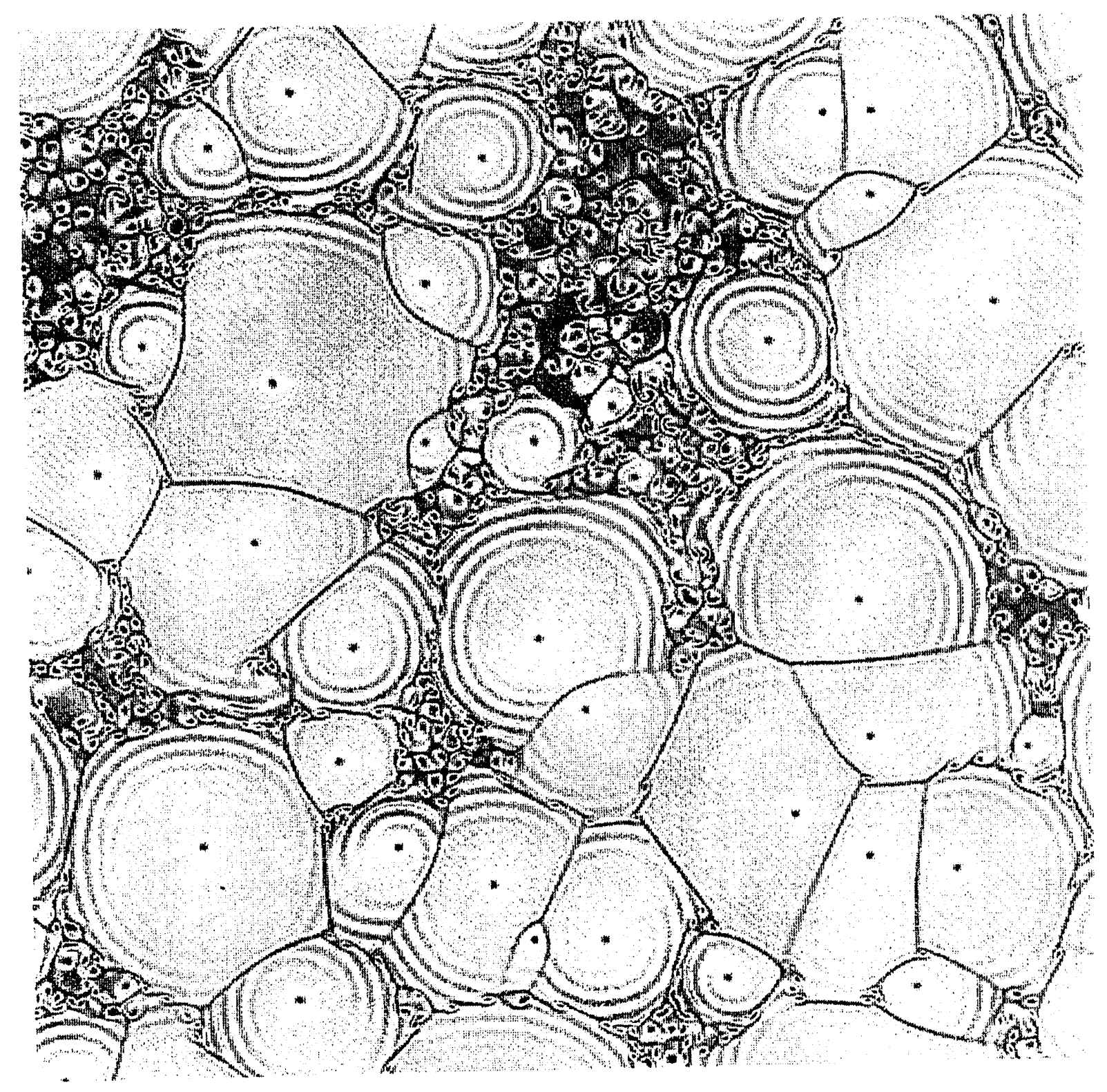

Figure 3.9: Intermediate stage of nucleation. Same parameters as Fig. 3.8. 


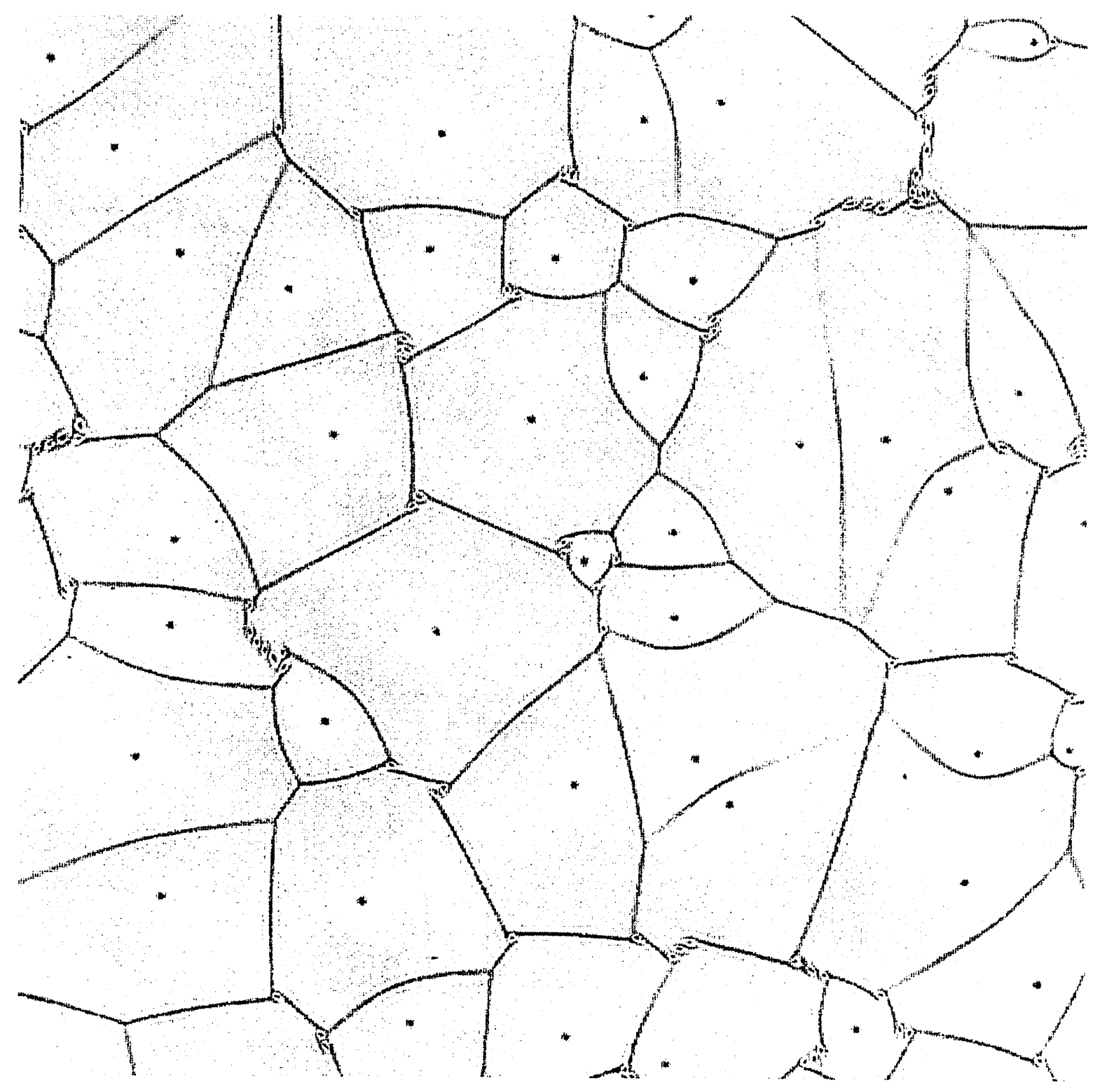

Figure 3.10: Nucleation is completed. Same parameters as Fig. 3.8. 
where some consequences of the nucleation picture are pursued.

\subsubsection{Parabola law: Model}

A very simple model consisting of expanding circular regions accounts for the parabola law. We assume that the radius of a vortex droplet increases with a constant velocity $v$, and that the density decays from $\rho_{\text {turb }}$ to $\rho_{\text {froz }}$ through the growth of a fixed, low density $\left(\rho_{f r o z}\right)$ of nucleation sites. The general idea is shown in the successive time frames of Fig. 3.11. In fact, the cartoon in Fig. 3.11 is somewhat too faithful to the phenomenon, since the model below assumes noninteracting vortices. The final frozen pattern in our simulations appears very much like the Voronoi (or Wigner-Seitz) cell construction of Fig. 3.11e.

Define $\Delta \rho=\rho_{t u r b}-\rho_{\text {froz }}, A_{\text {froz }}(t)$ equal to the area covered by the frozen phase at time $t$, and $L^{2}$ as the system size. Then

$$
\dot{\rho}=-\Delta \rho \dot{A}_{f r o z} / L^{2}
$$

where $r$ is the radius of any given droplet. Integrating this and matching the boundary conditions gives

$$
\rho=\rho_{t u r b}-\pi v^{2} \rho_{f r o z} \Delta \rho t^{2}
$$

Although the above model reproduces the quadratic law, it ignores some obvious features, such as the time-dependent density of nucleation sites. Such refinements should only affect the coefficients in (3.7). 


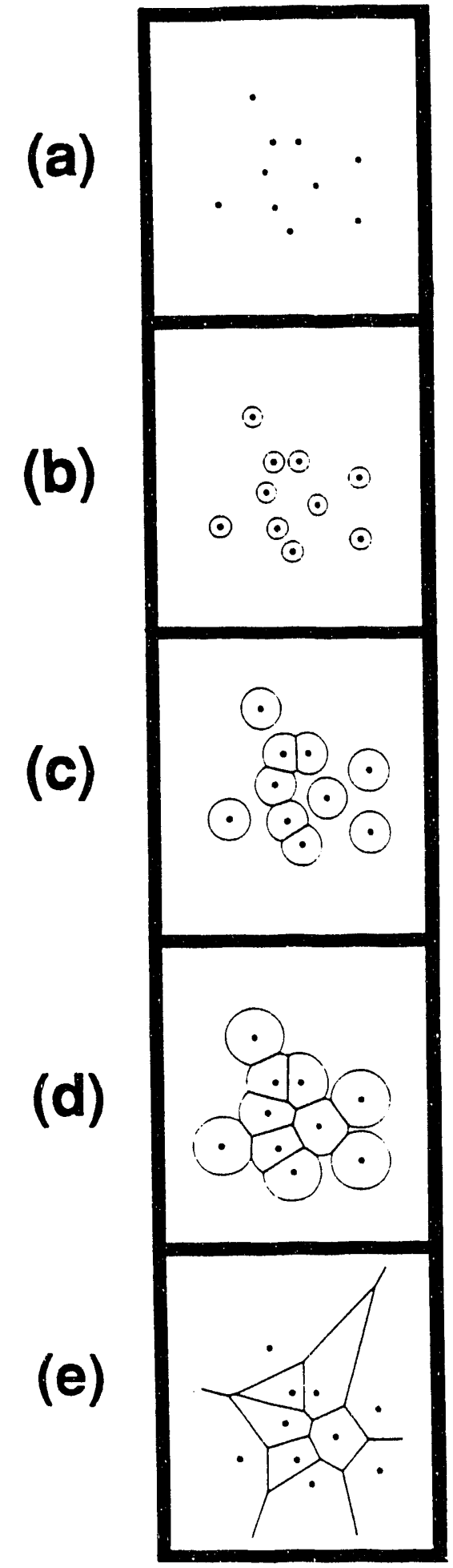

Figure 3.11: Cartoon of vortex droplet growth in five frames (a)-(e). 


\subsection{XY-type Decay}

\subsubsection{The exponent $\xi$}

For the special case $\alpha=\beta=0$ (the real Ginzburg-Landau equation), we present numerical evidence and general arguments to show that the vortex density follows a power law

$$
\rho \sim t^{-\xi}
$$

at late times with an exponent $\xi=3 / 4$. The generality of the arguments suggests that the exponent is universal for two-dimensional Coulomb-gas systems, including the important case of freely-decaying two-dimensional turbulence.

Let us begin with the numerical evidence for a power-law decay in the $\alpha=\beta=0$ system. Fig. 3.12 is our usual $\log$-log trace of density versus time. At early times, we again find that the density decays like $1 / t$ (i.e. $\xi=1$ ). The physical picture of a sea of vortices and antivortices pair annihilating and being pair-created was presented in Section 3.2. Fig. 3.13 displays the phase of $A$ at an early time. This result is also in agreement with the $X Y$ literature $[30,31]$, and with simulations of the scattering function by Mondello and Goldenfeld [32] (the latter speculate however that $\xi=1$ at very late stages). And, as in the CGL case, at later times, the vortices are more sparsely distributed (Fig. 3.14), making the annihilation rate non-trivial. Here we find numerically that the vortex decay follows the power law (3.8), with $\xi=0.74 \pm 0.02$ (Fig. 3.12). 


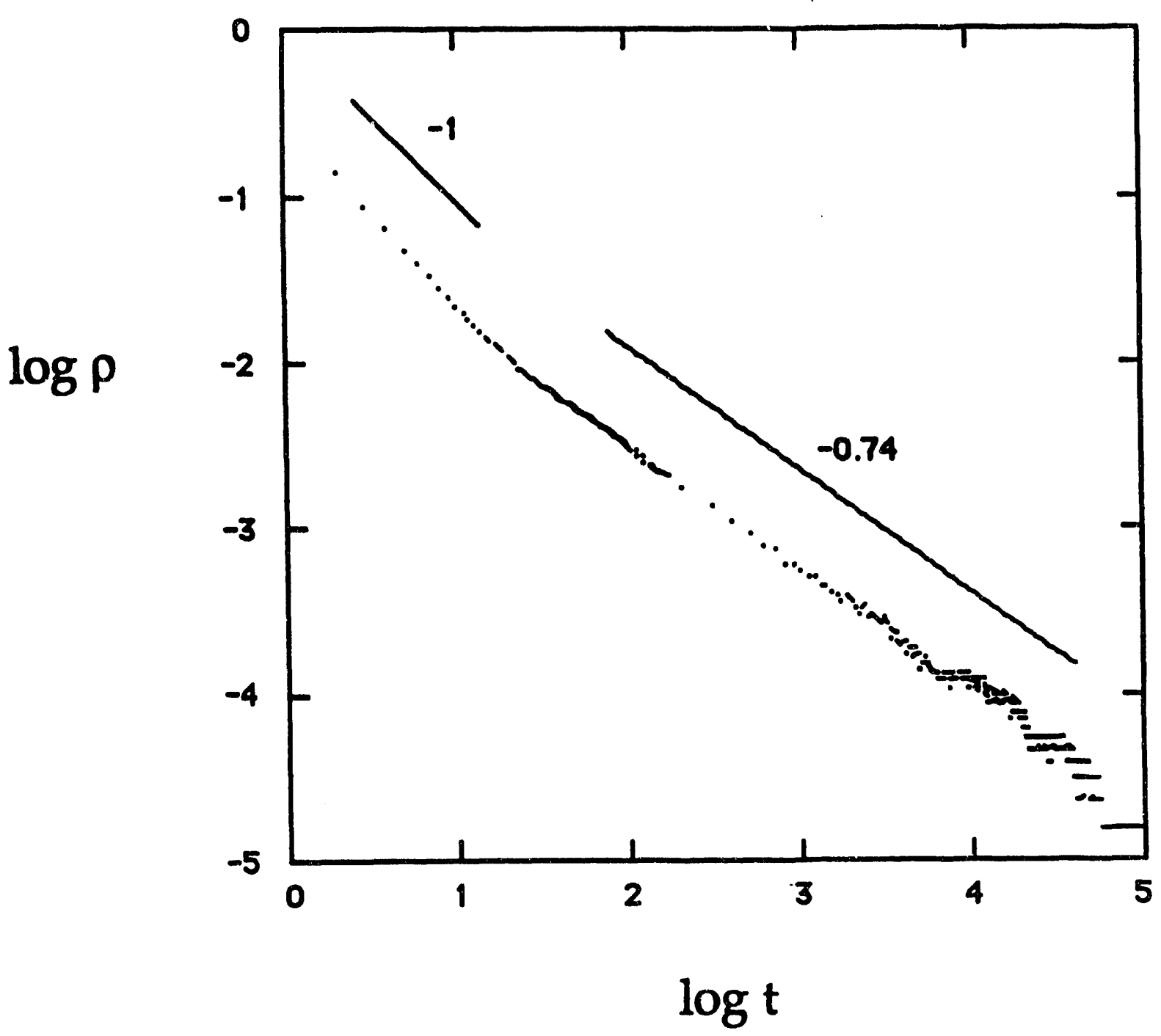

Figure 3.12: Log-log plot of density $\rho(t)$. Initial slope is compared with a slope of -1 . The asymptotic slope is found to be $\xi=0.74 \pm 0.02$. 


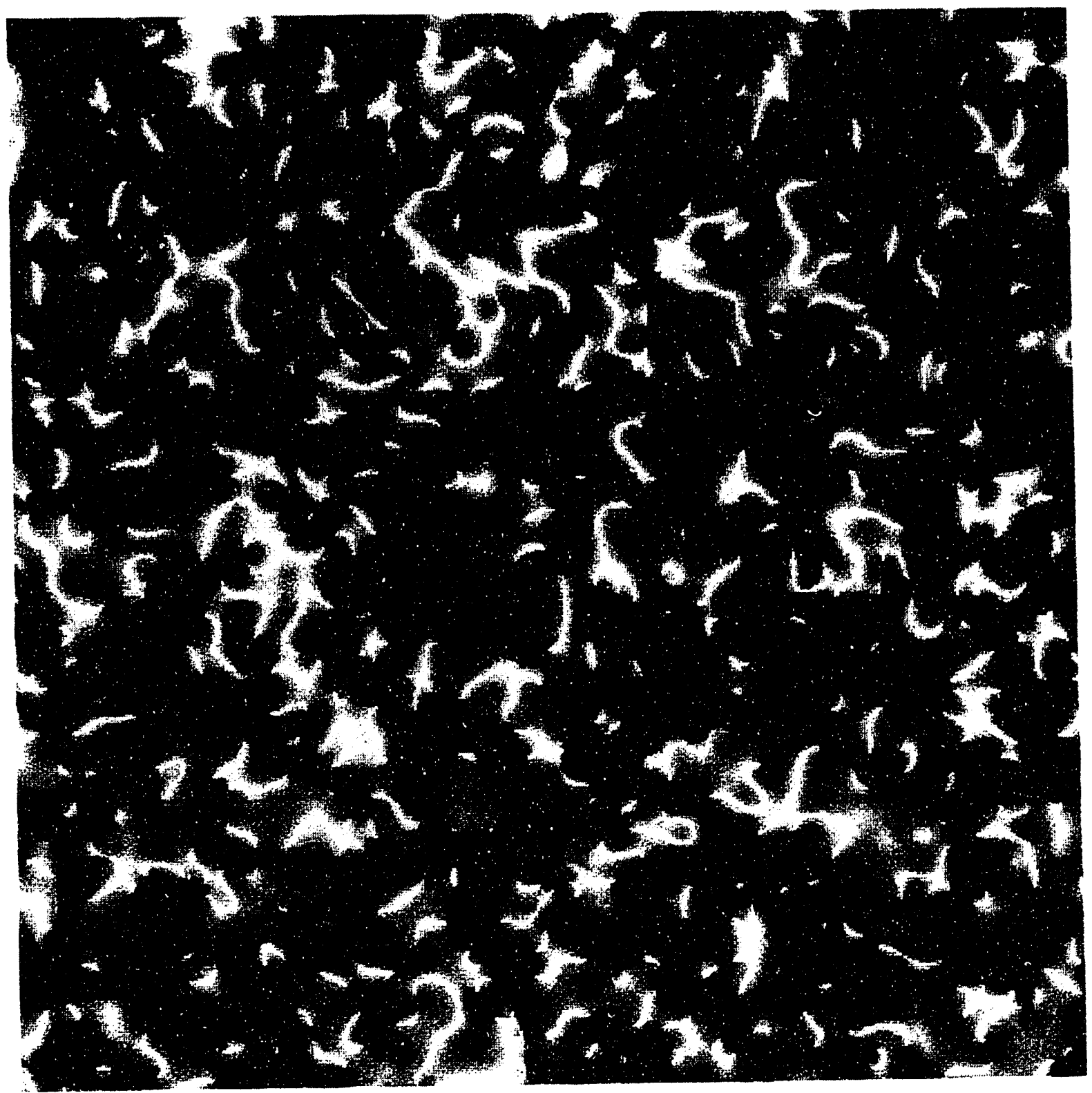

Figure 3.13: Phase field for the real Ginzburg-Landau equation at time $t=64$. Shown is a $380 \times 380$ section of a $512 \times 512$ lattice. 


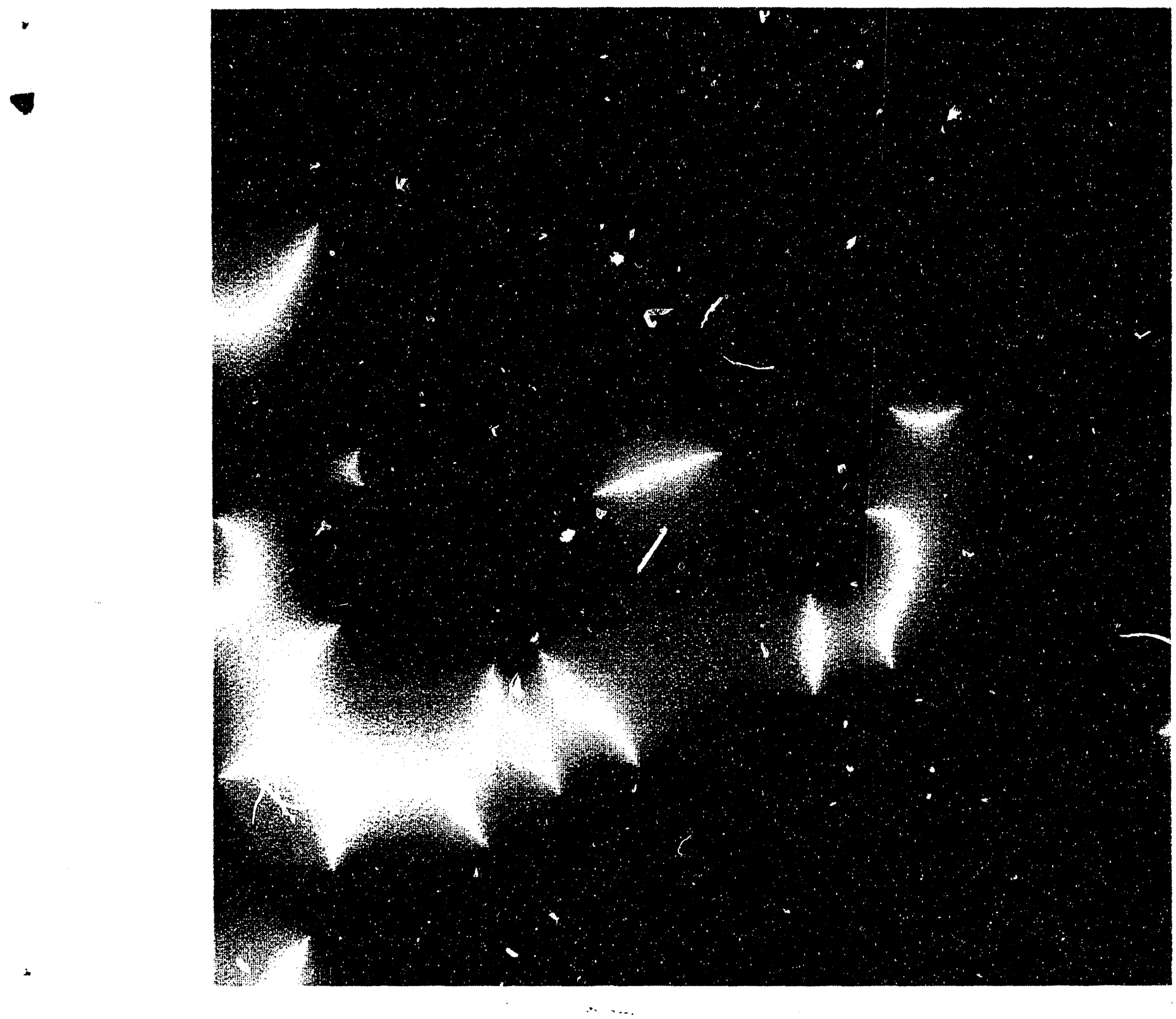

Figure 3.14: Phase field for the real Ginzburg-Landau equation at time $t=4096$. Same view as rig. 3.13 . 


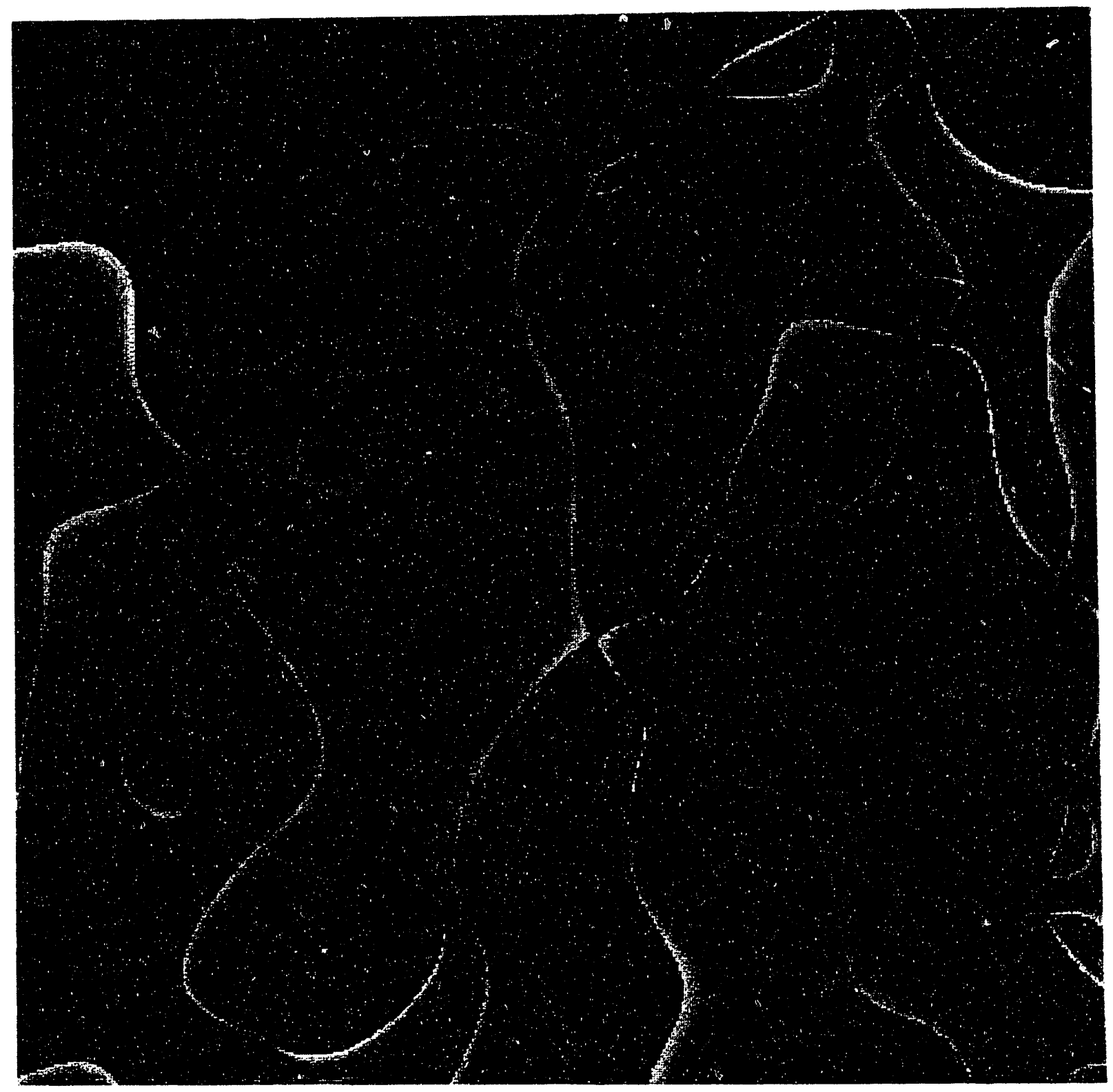

Figure 3.15: Phase portrait, as in Fig. 3.14, with phase divided into 20 bands to bring out the electrostatic analog. 


\subsubsection{Annihilation scale}

A length scale $R$, characteristic of time $t$, can be extracted by considering the average initial separation between vortices and antivortices that annihilate at time t. In Fig. 3.16, pairs of vortices and antivortices that later annihilate, are linked by straight lines, and labeled with their annihilation time. At late times (low density), the vortices and antivortices interact via a logarithmic pair potential $\Phi\left(\mathbf{r}_{i}, \mathbf{r}_{j}\right)=$

$-k \log (r)$, where $r=\left|\mathbf{r}_{i}-\mathbf{r}_{j}\right|$. The dissipative equation of motion, $\dot{r}=-\frac{\partial \Phi}{\partial r}$, relates the separation $R$ between a vortex and antivortex to their annihilation time $t$,

$$
R \sim t^{1 / 2}
$$

Computation of annihilation times for our system of many XY vortices on a $128 \times$ 128 lattice are in agreement with (3.9) (Fig. 3.17).

\subsubsection{Continuous ordering}

The theory of continuous ordering [33] provides another reason to expect a dynamical length scale $R(t)$ that increases according to (3.9). The vortex dynamics can be viewed as the non-equilibrium phase ordering arising from an instantaneous quench from a high-temperature to a zero-temperature configuration. One could arbitrarily divide the phase $\phi(\vec{r}, t)$ of $A$ into two states by assigning +1 to sites with $\operatorname{Im}(A)>0$, and -1 to sites with $\operatorname{Im}(A)<0$. In this way, the complex order parameter is transformed to an up-down spin. The vortices and antivortices lie on the $+1 /-1$ interface (Fig. 3.18).

With no locally-conserved order parameter, (3.9) is predicted by the LifshitzAllen-Cahn theory $[33,34]$. 


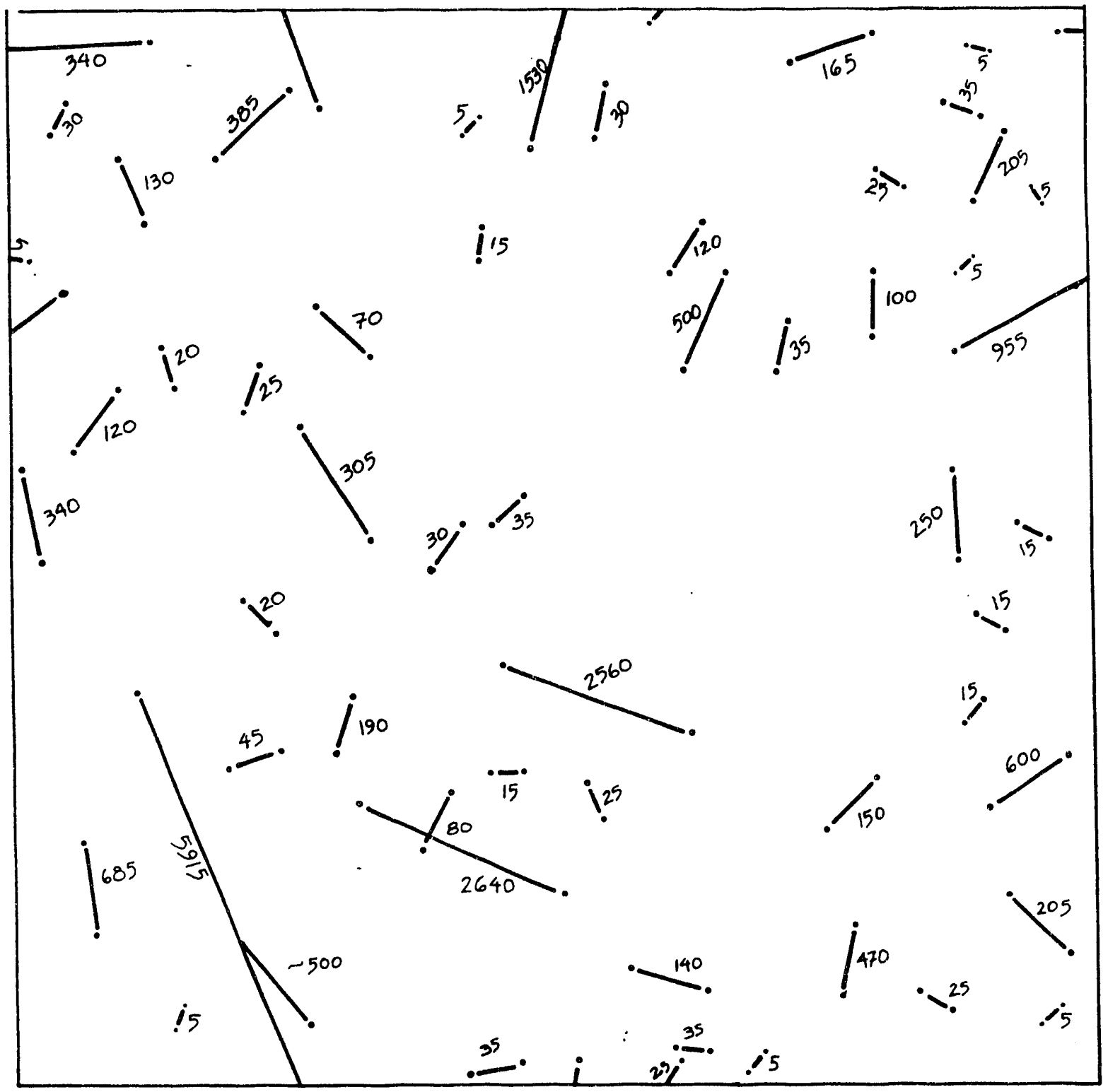

Figure 3.16: Vortex-antivortex pair positions at $t=30$. Straight lines connect the pairs and are labeled by their subsequent time of merger. Lattice size is $128 \times 128$. 


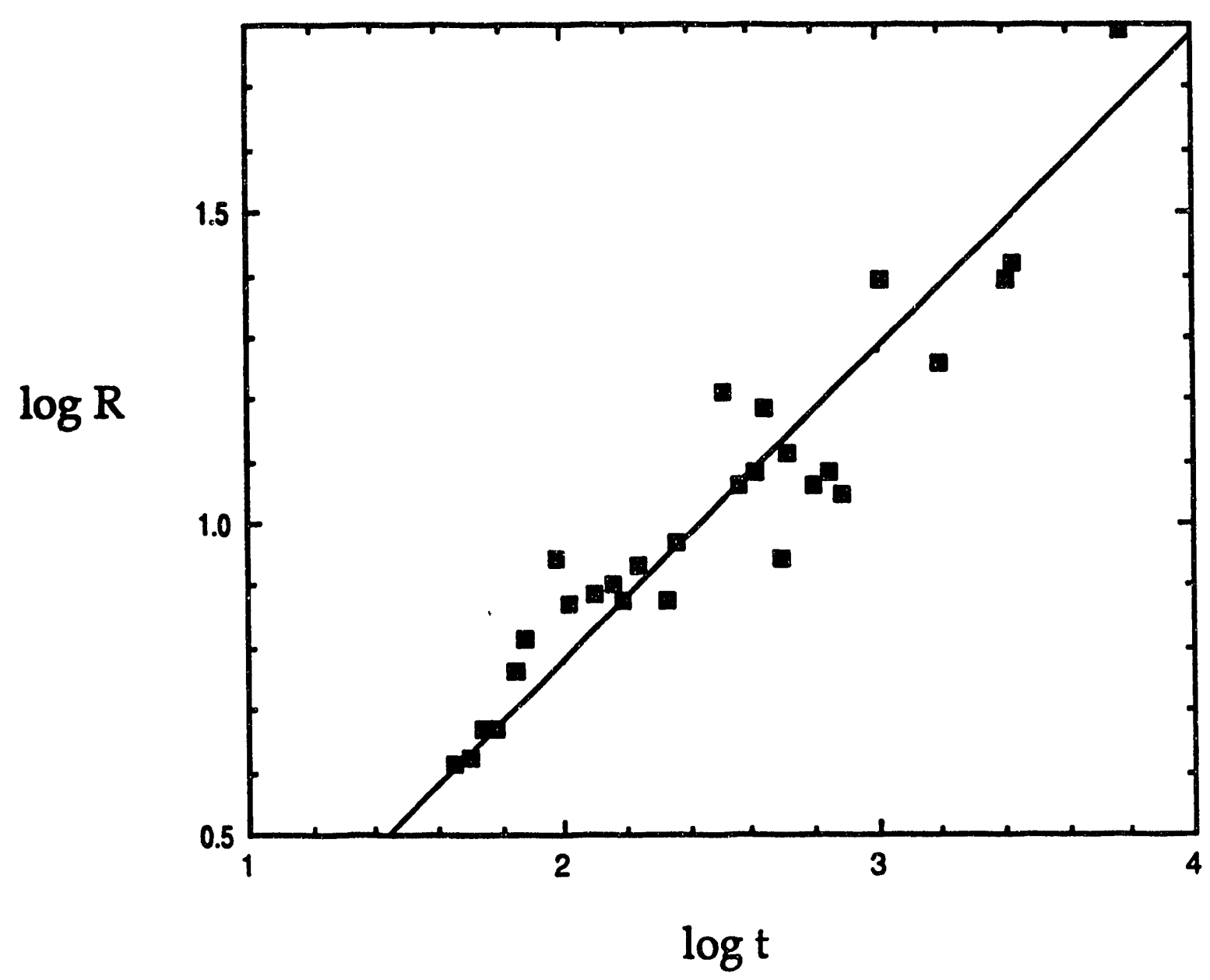

Figure 3.17: $\log$-log plot of vortex-antivortex separation $R$ (at time $t=30$ ) versus annihilation time. 


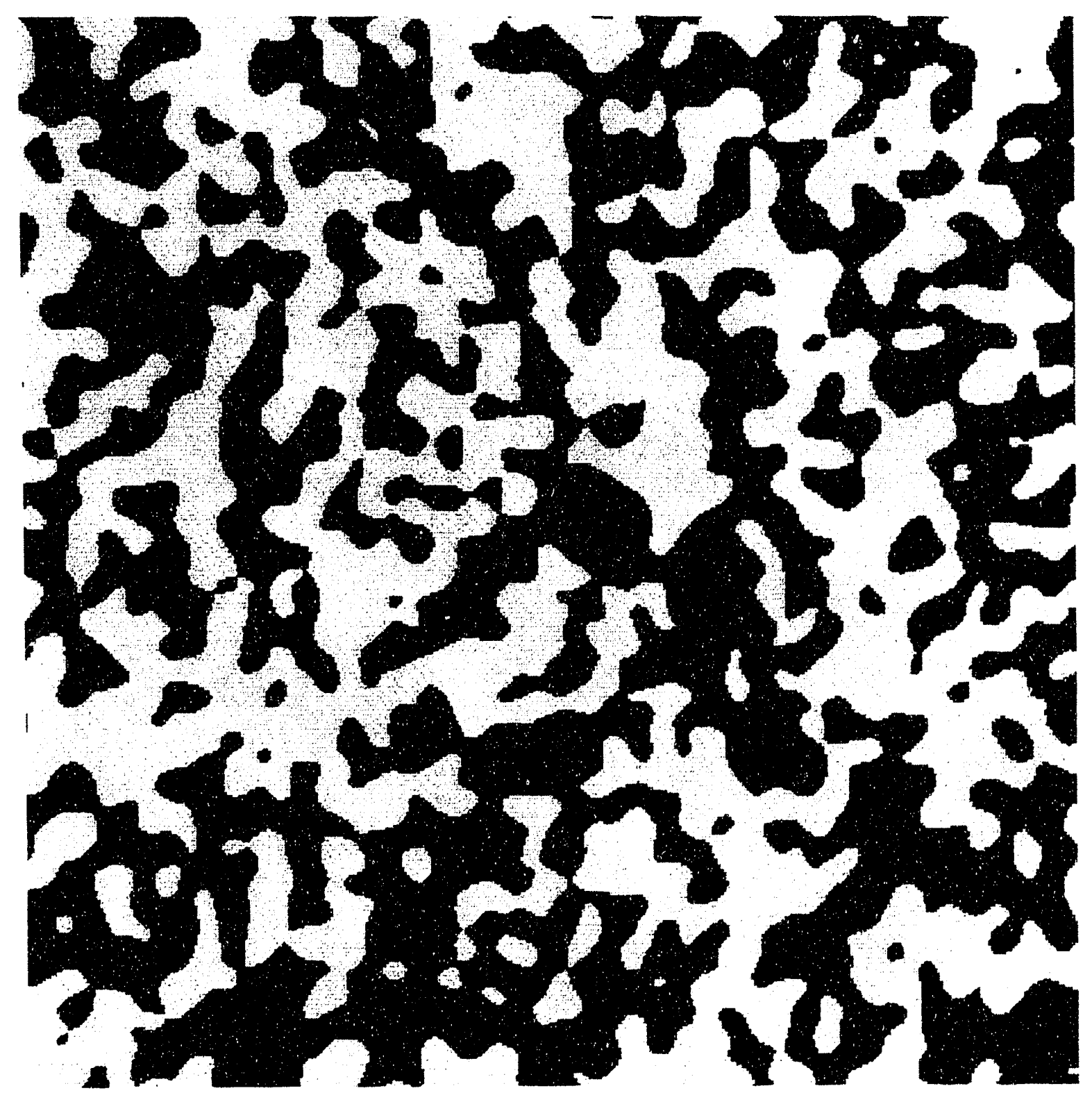

Figure 3.18: Phase $\phi$ divided into two states by assigning +1 (yellow) to sites with $\operatorname{Im}(A)>0$, and -1 (blue) to sites with $\operatorname{Im}(A)<0$. The vortice and antivortices must lie on the interface. Time $t=64$. 

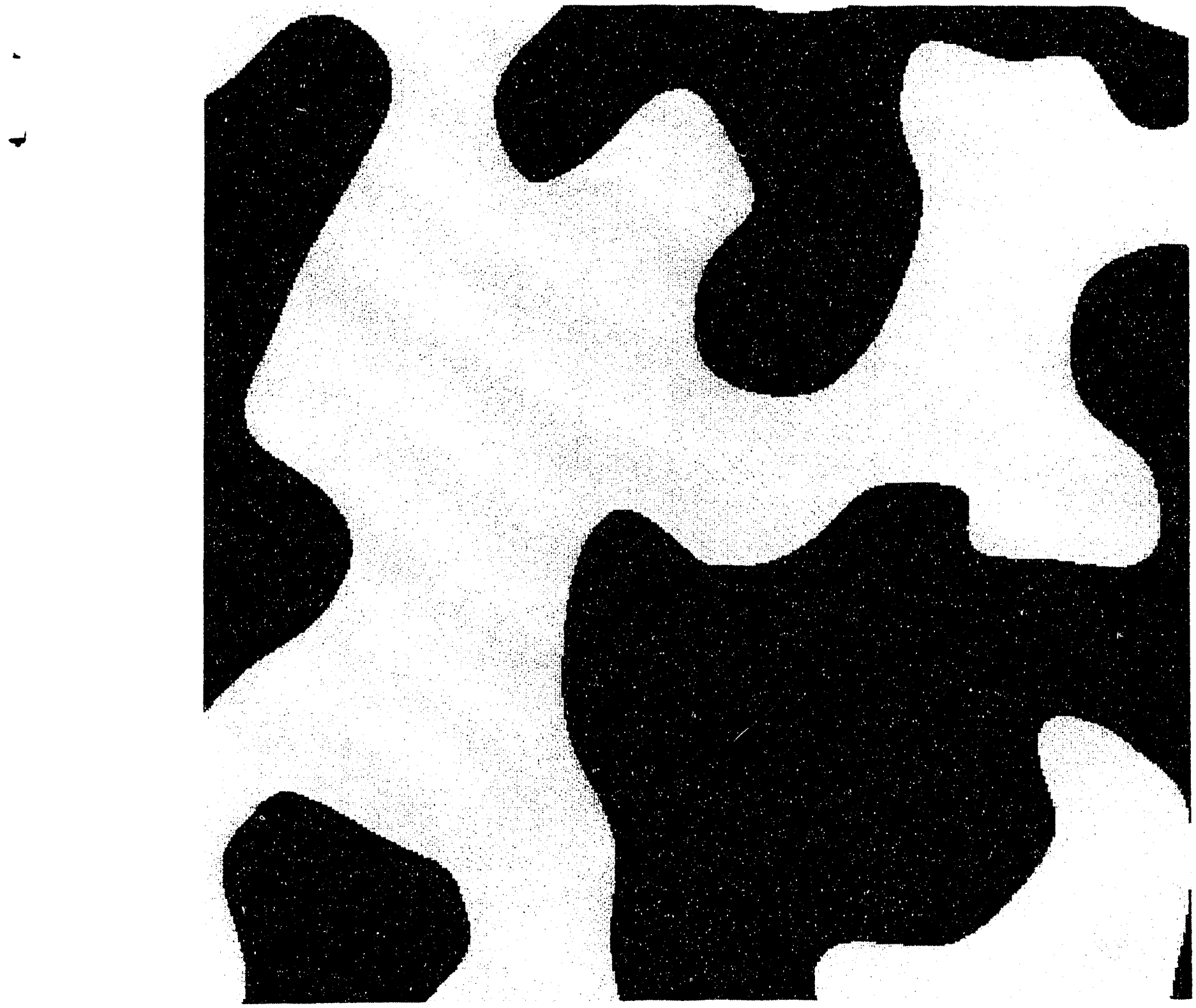

Figure 3.19: Phase plot with same parameters as previous figure. Time $t=4096$. 


\subsubsection{Interaction volume}

The decay can be understood in terms of the dynamical length $R(t)$, which we associate with an interaction volume $[35,36]$ : After a time $t$, all vortex-antivortex pairs within an area $A \sim R(t)^{2}$ have had a chance to annihilate. The number of remaining vortices $\rho(t) A$ equals $\delta\left(\Delta \phi_{A}\right) / 2 \pi$, where $\delta\left(\Delta \phi_{A}\right)$ is the standard deviation of the circulation $\Delta \phi$ over the perimeter enclosing the area $A$.

One might reason $[37,38]$ that because of the presence of free vortices in the initial high-temperature regime, the variance of $\Delta \phi_{\mathcal{A}}$ should scale like the area of $\mathcal{A}$. Though intuitively plausible, this 'area law' is incorrect. Based on a (hightemperature) uniform distribution of mutually-independent phase differences (mod $2 \pi$ ) between neighboring sites, Dhar [38] showed that, for large areas (late times), the variance of $\Delta \phi_{\mathcal{A}}$ at all temperatures is proportional to the perimeter of $\mathcal{A}$, i.e. $\delta^{2}\left(\Delta \phi_{\mathcal{A}}\right) \sim R$. Thus,

$$
\rho R^{2} \sim \delta\left(\Delta \phi_{A}\right) \sim \sqrt{R}
$$

In other words, $\rho \sim R^{-3 / 2}$, and (3.9) yields the exponent $\xi=3 / 4$.

\subsubsection{Freely-decaying turbulence}

The general arguments above suggest that $\xi$ is universal. This conclusion is further supported by recent numerical and experimental results obtained for freely-decaying two-dimensional turbulence. These results also point to universal vortex decay, from highly turbulent to laminar, vortex-free flows. The evolution of vorticity is here given by the fluid-dynamical equation

$$
\partial_{t} \omega+J(\psi, \omega)=\nu \nabla^{2} \omega, \quad \omega \equiv-\nabla^{2} \psi
$$


where $\omega$ is the scalar vorticity, $\psi$ the stream function, $\nu$ the kinematic viscosity, and $J(\psi, \omega)=\partial_{x} \psi \partial_{y} \omega-\partial_{x} \omega \partial_{y} \psi$ the Jacobian. For this system, McWilliams [39] found the exponent $\xi$ to be $\xi \simeq 0.71$. In later works $[40,41]$, the value is variously reported $\xi \simeq 0.72-0.75$, and preliminary results pointed to a universal $\xi$ value for a whole hierarchy of freely-decaying turbulence models. A recent turbulence experiment by Tabeling et al. [42] yielded $\xi=0.7 \pm 0.1$, in support of this conjecture.

In should be pointed out that the initial separation $R \sim \rho^{-2 / 3}$ between vortices that later merge (annihilate) differs from the average distance $l \sim \rho^{-1 / 2}$ between vortices (of both signs). It is a consequence of this, and not of anomalous diffusion, that close encounters between vortex pairs occur less frequently than expected from the random motion oi (sign-less) vortices [39]. 


\section{Chapter 4}

\section{Transient Turbulence}

\subsection{Transient Overview}

Of fundamental interest is the occurrence of transient turbulent states near the transition line that separates the frozen states from the turbulent states. In this chapter, we present present analytical and numerical results that explain the transient turbulent dynamics. Just below the transition to turbulence, we observe that turbulent states break down by the nucleation and growth of single-vortex droplets, leading to frozen states of stationary vortices. We derive a relation between nucleation time and radius, and determine their dependence on the distance to the turbulence transition line. 


\subsection{Critical line}

\subsubsection{Local density minimum}

To understand better the occurrence of transients, let us return to the density traces of Fig. 3.2. In that plot, the density was followed for times of order $10^{5}$ for a sequence of $\alpha$ values $(0,0.25,0.6,0.75,0.8,2)$ at fixed $\beta(\beta=-1)$. The traces corresponding to $0.6,0.75$, and 0.8 were associated with transient states of turbulence.

Through a comparison of density traces with their corresponding amplitude portraits, it was verified that the existence of the local density ininimum (at roughly $t=100)$ signals the beginning of a turbulent steady state (transient or otherwise). When this density minimum vanishes (as a function of $\alpha$ ), so does the transient turbulence. We call this critical $\alpha$ value, where the transient state first appears, $\alpha_{c}$, since it marks the boundary between two qualitatively different many-vortex dynamics. As an example, Fig. 3.2 shows that $\alpha_{c}(\beta=-1)$ lies between 0.25 and 0.6. A finer sweep through this particular $\alpha$ range puts this critical value at $\alpha_{c}=0.57 \pm 0.03$. Extending the analysis to other values of $\beta$, we find the curve $\alpha_{c}(\beta)$, plotted in Fig. 4.1 (as the dotted line with error bars) alongside the turbulence transition and Benjamin-Feir lines.

\subsubsection{Linear stability analysis}

The value $\alpha=\alpha_{c}(\beta)$ can be estimated by linear stability analysis (solid-circle line (ECK) in Fig. 4.1). The stability (with respect to small perturbations) of planewave solutions of the form $A(\mathrm{k})=R(\mathrm{k}) \exp [i(\mathrm{k} \cdot \mathrm{x}-\omega(\mathrm{k}) t)]$, can be worked out as 


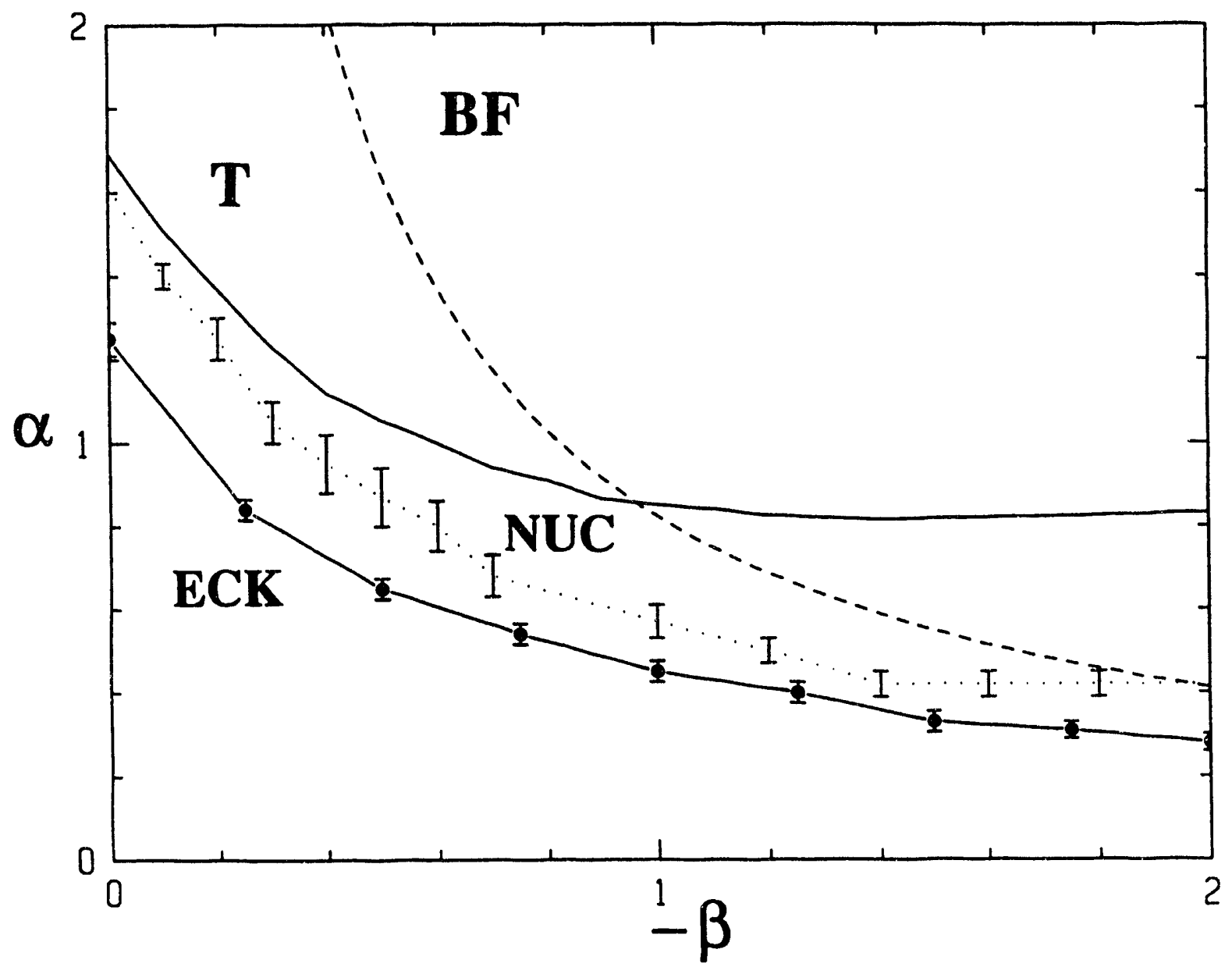

Figure 4.1: Phase diagram for $0 \leq \alpha \leq 2$, and $-2 \leq \beta \leq 0$. (NUC): Line $\alpha_{c}(\beta)$ below which nucleation is no longer observed (dotted, with error bars). (ECK): Eckhaus instability line (solid-circle, with error bars). (T): Transition line to vortex turbulence (solid). (BF): Benjamin-Feir line (dashed). 


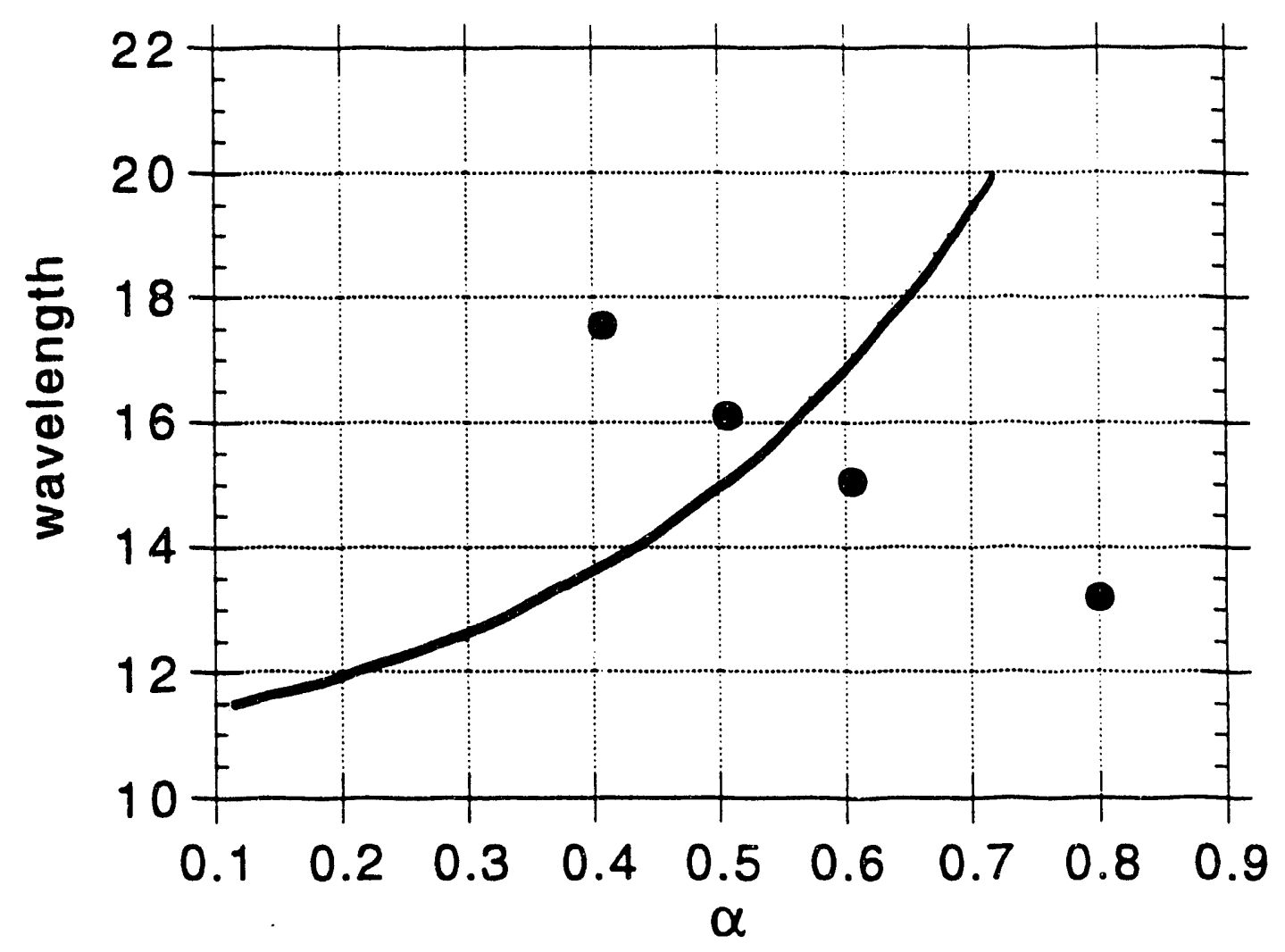

Figure 4.2: Linear instability threshold (solid line) and selected wavelengths (circles), plotted as wavelength versus $\alpha . \beta=-0.75$.

an extension to the $\mathrm{k}=0$ analysis of Section 1.4.1. Bohr et al. [16] have done this for the case of the coupled-map construction. As an example of their analysis, the bold curve in Fig. 4.2 is a plot of the smallest unstable $\alpha$ for a fixed wavelength $\lambda=2 \pi /|\mathbf{k}|$. (In this example, $\beta=-0.75$.) The circles in the figure correspond to the "natural" wavelengths found in Table 2.1. The intersection of the two curves provides an estimate of $\alpha_{c}$. In other words, for $\alpha>\alpha_{c}$, the plane-wave solution with the selected, parameter-dependent wavelength is linearly unstable (Eckhaus: instability (43]). From Fig. 4.2, we estimate $\alpha_{c}=0.55 \pm 0.01$, in good agreement with the observation of the onset of metastability reported in the previous section. 
As the Fig. 4.1 shows, the linear instability estimate is always lower than the one found from nucleation. This is to be expected since the magnitudes of the eigenvalues determining the instability grow very slowly with $\alpha$ above the instability line, and the corresponding nucleation times $T$ become too short to be resolved. Also one must keep in mind the fact that the stability line is calculated assuming a plane-wave state with the selected wavelength - the existence of the vortex cores is not taken into account.

\subsection{Near the Transition Line}

\subsubsection{Absolute instability}

The Eckhaus instability is of convective type: although the linear instability signifies exponential growth, this growth is only observed in a frame moving with the group velocity. This was noted recently by Aranson et al. [28], who further conjectured (with support from simulation) that the onset of turbulence $\alpha=\alpha_{0}$ is close to the onset of absolute instability, where the exponential instability takes place even in the rest frame. This conjecture has been checked for our coupled-map representation by extending the stability analysis to the complex plane and computing the eigenvalue $\chi$ that corresponds to the saddle point [44]. In fact one finds that $\chi$ passes through 1 very near the onset of turbulence and we now show how this can be used to estimate the transient lifetime $T$ and find its dependence on the parameters. 


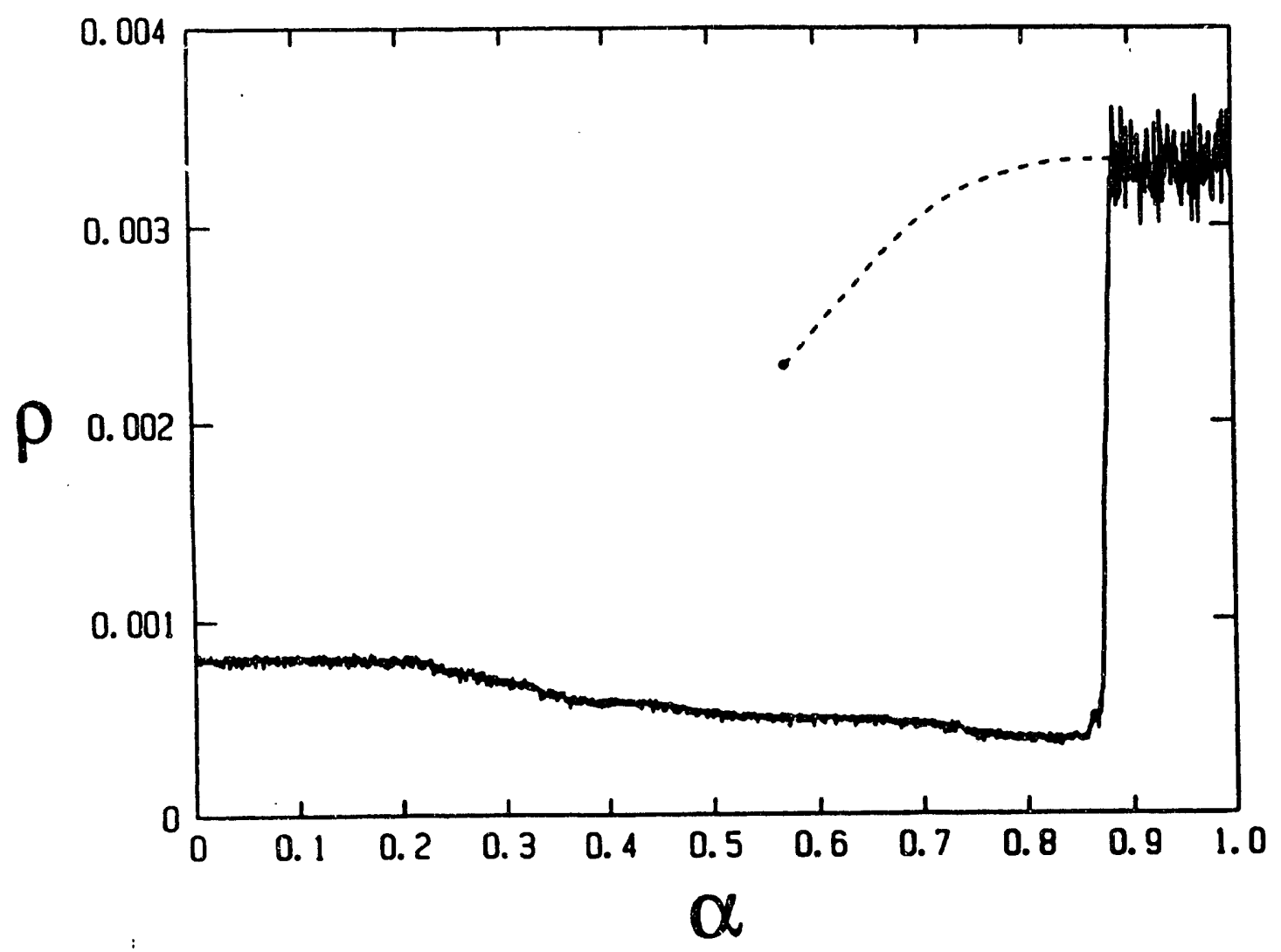

Figure 4.3: Vortex density versus $\alpha(\beta=-1)$, as in Fig. 2.15, with addition of the density curve for the metastable (transient) states. The - represents the onset of transient turbulence, and corresponds to the dotted line (NUC) in Fig. 4.1.

\subsubsection{Nucleation time}

In previous sections, we saw that for alpha above the crit:2] curve $a_{c}$, a density minimum occurs at $T_{s}$, after which the density assumes a roughly constant value. In Chapter 3, we associated the end of this transient with the nucleation of vortex droplets, and hence refered to the transient as a metastable state. We can keep track of these metastablı states by plotting their density as a furiction of $\alpha$ (keeping $\beta$ fixed), and superposing this plot onto our earlier Fig. 2.15.

We define the transient lifetime $T$ so that after time $T_{s}+T$, the system just be- 
gins its decay to the frozen state. More exactly, the transient time $T$ was computed by determining when the vortex density had decayed by two standard deviations from its average value in the metastable turbulent state. We have found that the average transient lifetime diverges as the transition line is approached [18]. (This last result was also found in [24], where the transient lifetime was identified by

measuring the Lyapunov exponent.) We can think of the time $T$ as a nucleation time - the time required to nucleate a growing droplet.

\subsection{Geometrical Nucleation}

\subsubsection{Outer perturbations}

A nucleating droplet contains only one vortex (spiral wave). A necessary condition for the droplet to form is that this central vortex survives outer turbulent perturbations for a time period $\Delta t$ large enough to locally stabilize the spiral wave. A perturbation decays like $\chi^{t}$, so the time needed is $\Delta t \sim|\log \chi|^{-1}$. If $v$ denotes the (average) turbulent vortex velocity, we are led to the following condition: for a droplet to form, the distance $R$ from the 'nucleating' vortex to the closest perturtation center (outer vortex) must he at least

$$
R=v \Delta t \sim v|\log x|^{-1}
$$

For fixed $\beta$, an expansion to lowest order in $\alpha$ yields $v \sim v_{0}$ and $\log \lambda \sim\left(a-a_{0}\right)$, where $v_{0}$ is the turbulent velocity at the instability. Thus, by (4.1),

$$
R \sim\left(\alpha_{0}-\alpha\right)^{-1}
$$




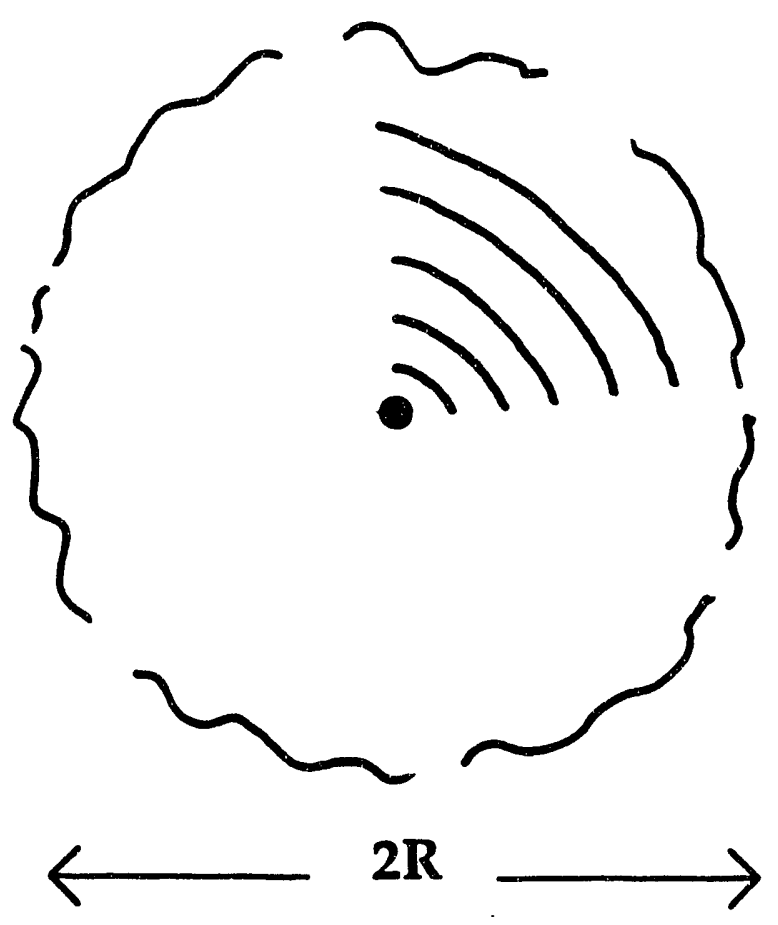

Figure 4.4: Vortex droplet with amplitude waves, buffetted by outer perturbations. 


\subsubsection{Divergence}

The nucleation time $T$ is the time it takes for a nucleating droplet to form. From the considerations above, $T$ is the time we have to wait before a single vortex somewhere becomes separated from all other vortices by a distance larger than $R$. If we assume that the vortices move randomly, then $T \sim 1 / p$, where $p$ is the probability that an area $\pi R^{2}$, that contains a single vortex, exists in one realization of randomly-distributed vortices. In each region of size $\pi R^{2}$, the probability $p_{1}$ of finding exactly one vortex is given by the binomial form

$$
p_{1}=N r(1-r)^{N-1}, \quad r \equiv n / N
$$

where $n=\rho \pi R^{2}$, and $N$ is the total number of vortices. Moreover, $1-p=$ $\left[1-p_{1}\right]^{N / n}$. For $1<<n<<$, we have $p \sim N e^{-n}$, and thus by (4.2)

$$
\log \left(T / T_{N}\right) \sim\left(\alpha_{0}-\alpha\right)^{-2}
$$

where the time scale $T_{N}$ decreases with $N$. (The size dependent time scale $T_{N}$ is associated only with the time needed to form the first droplet, not with the time to reach the final frozen state.)

We have determined $T(\alpha)$ in our simulations (for $\beta=-1$ ), averaged over five runs. As shown in Fig. 4.5, the analytic form (4.4) fits the data quite well. Furthermore, a two-variable fit, gave best agreement for $\alpha_{0}(-1)=0.84 \pm 0.005$ and and exponent (for $\alpha_{0}-\alpha$ ) of $-1.97 \pm 0.10$, in excellent agreement with our theory [18]. 


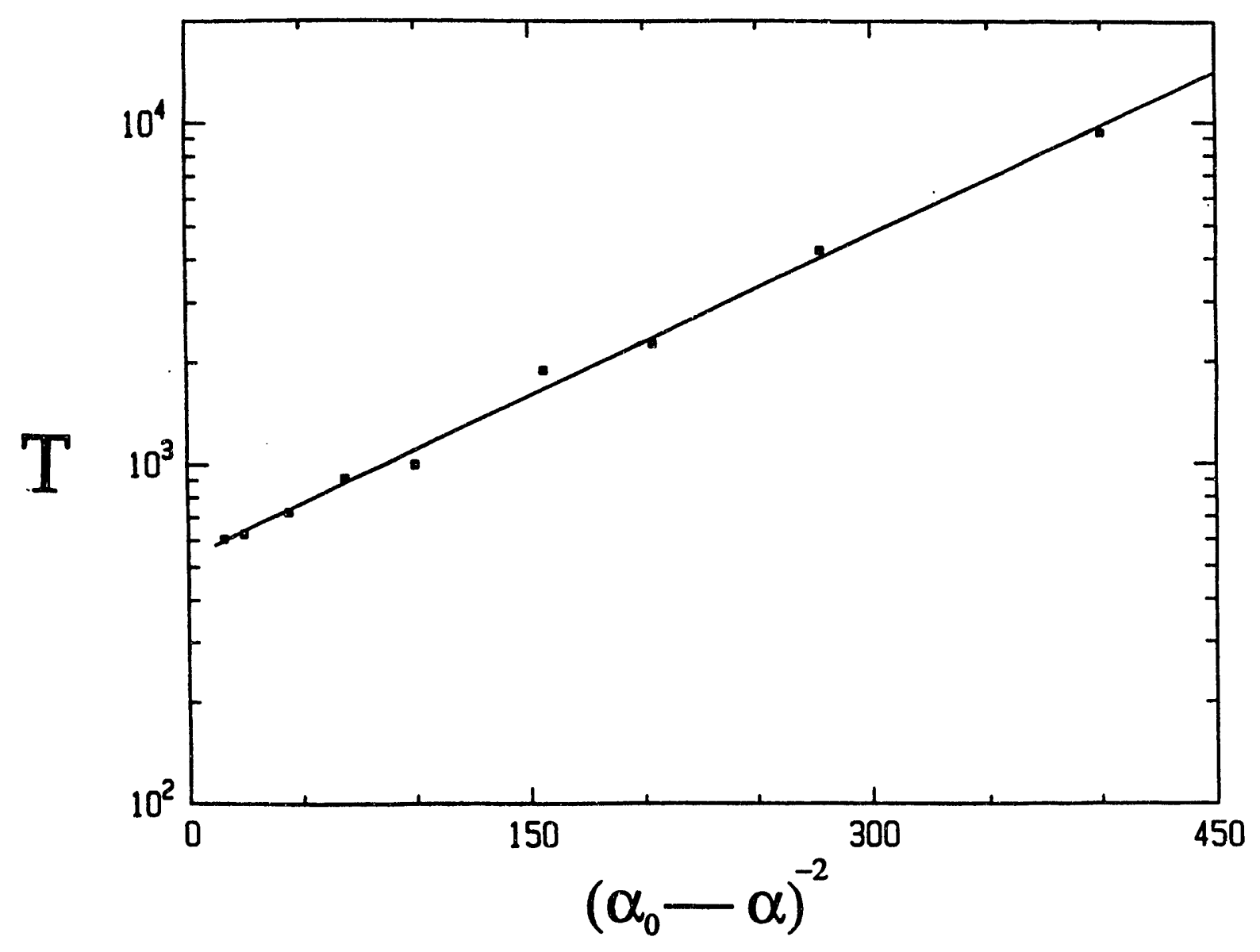

Figure 4.5: Semilogarithmic plot of average nucleation time $T$ versus $\left(\alpha_{0}-\alpha\right)^{-2}$ (for $\beta=-i$ ). Svstem size is $512 \times 512 . \alpha_{0}(-1)=0.85$. 


\section{Chapter 5}

\section{Conclusions and Extensions}

\subsection{Conclusions}

We briefly summarize the main results of this thesis:

From random initial conditions, we found a low-density frozen state of stationary vortices.

We located the transition to vortex turbulence by measuring the vortex density and observing an order-of-magnitude jump from its value in the frozen state. We presented a phase diagram showing the transition line between frozen and turbulent states. A metastability curve corresponding to transient turbulence was also located in the phase diagram.

We explored the properties of metastable turbulence which precedes a range of asymptotic frozen states. We observed that they break down by the random appearance and growth of single-vortex droplets, and gave evidence for this nucleation model.

Finally, we measured the lifetime of these transient states as the turbulence 
transition is approached, and found it diverges according to a simple law. We explained this law through the nucleation model.

\subsection{Extensions}

\subsubsection{Suprises in quadrant III}

Back in Section 1.4.2, we restricted our subsequent attention to the quadrant of the phase diagram having $\alpha \geq 0$ and $\beta \leq 0$ (quadrant II). Later, we saw that the turbulent transition line and the Benjamin-Feir line were quite different - not suprisingly, the stability of the homogeneous state was not a good predictor for the many-body vortex states. Thus, the study of a Benjamin-Feir stable quadrant, quadrant I $(\alpha \geq 0, \beta \geq 0)$ for instance, is an appropriate extension of the present thesis.

As a quick sortie into this unexplored region, imagine beginning from a frozen vortex state at $\alpha=0, \beta=-1$, and lowering $\alpha$ at a fixed rate. This will take us into quadrant III, which we know from the symmetries of the CGL to be equivalent to quadrant I. We have performed only relatively fast $\alpha$ sweeps $\left(\dot{\alpha} \approx 10^{-2}\right)$ into quadrant III. This procedure has uncovered a turbulent region at $\alpha \approx 5$ (for $\beta=$ $-1)$. The turbulence is dominated by stringy structures of zero amplitude and finite length, which wriggle back and forth, shedding other strings like wavefronts, and dissipating at their ends, leaving a vortex behind. The configuration is then one of a finite density of vortices interspersed between the rapidly moving turbulent filaments.

The particular instability excited by these fast sweeps is equally fantastic. The 


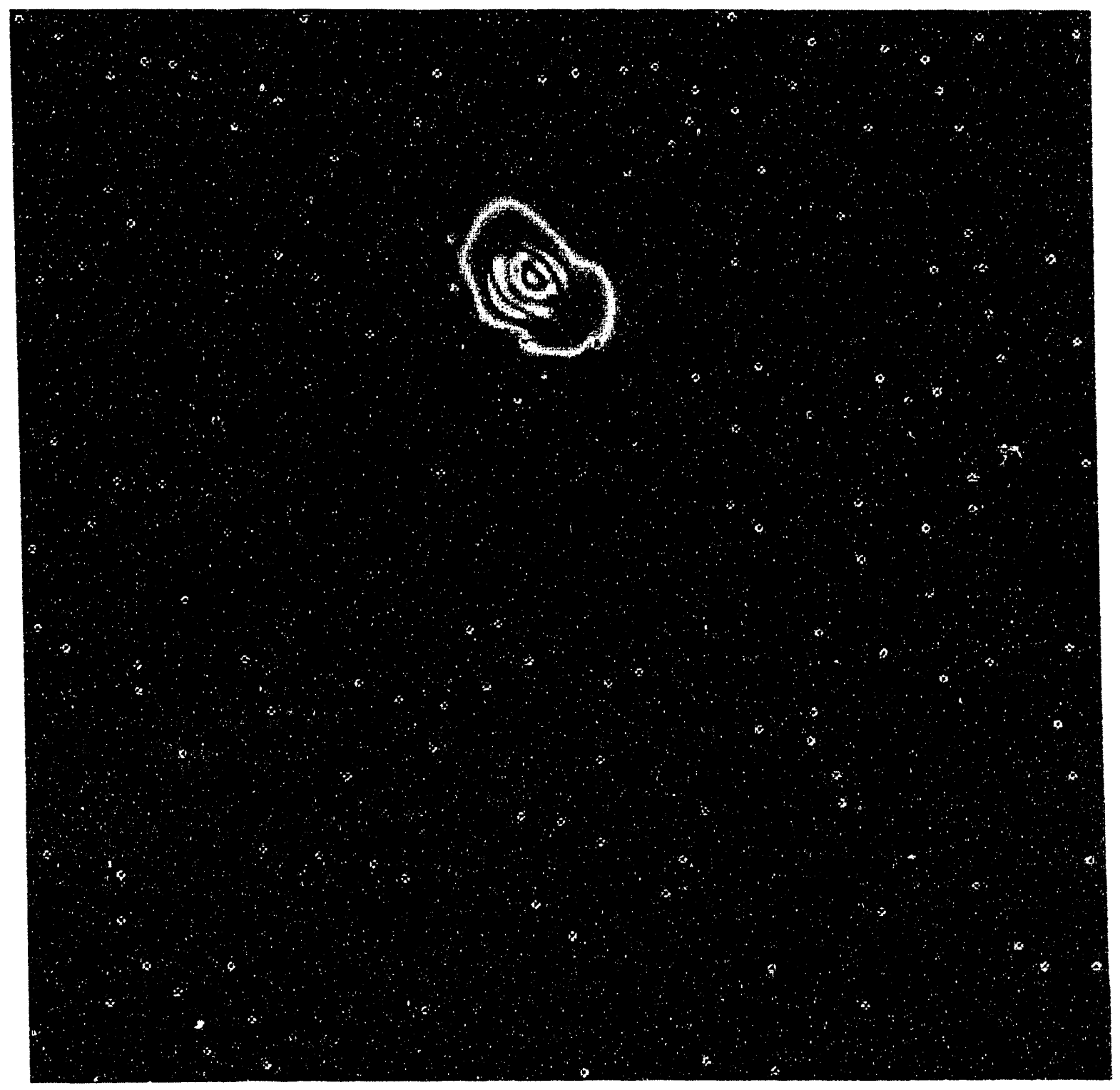

Figure 5.1: "Spindle body" resulting from a recent vortex-antivortex annihilation event (amplitude portrait). Parameters are $\alpha=-5.5, \quad \beta=-1$; system size is $375 \times 375$. 


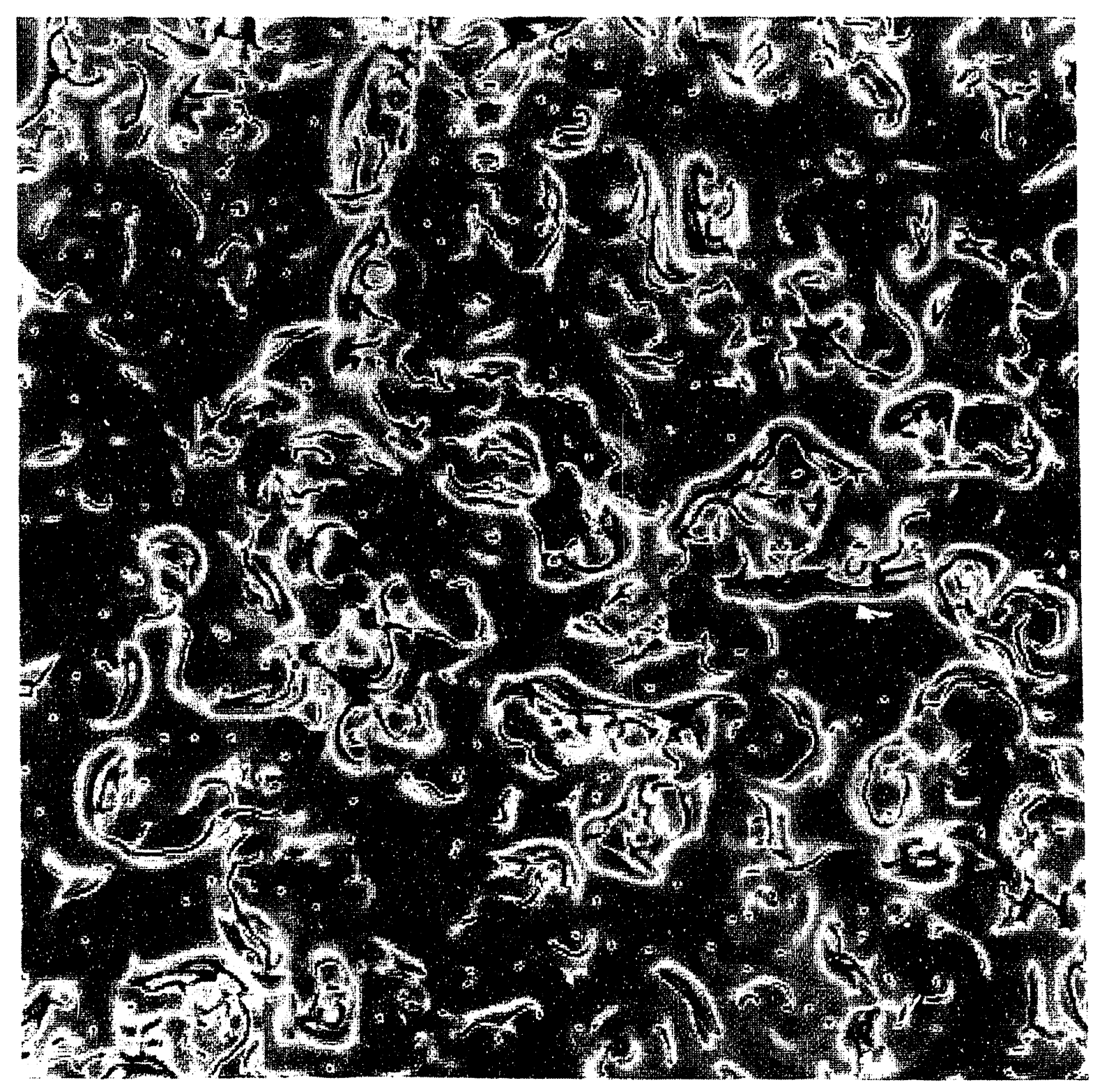

Figure 5.2: Filamentary turbulence. Same parameters as Fig. 5.1. 
entire turbulent pattern is initiated by a single vortex-antivortex annihilation. When a single wavefront from this annihilation event decreases in amplitude to zero amplitude, a short curved arc of zero amplitude results. Very quickly. a small. but steadily expanding, "spindle body" (Fig. 5.1) is created by the motion and wavefront shedding of the curved segment. The edges of this turbulent region expand at a high velocity, and soon the entire region is filled with this filamentary turbulence (Fig. 5.2).

\subsubsection{Vortex lines in three dimensions}

Of great physical and chemical relevance is the behavior of the CGL equation in three dimensions. (See reference [25] ior a phenomenological analysis of defects in three-dimensional excitable media.) The coupled-map scheme described in Section 1.3 is applicable to lattices in any dimension. For $d=3$, we must simply modify the discrete Laplacian in (1.28) by averaging now over the 6 nearest neighbors to a site of a cubic lattice. Fig. 5.3 shows the result of simulating the CGL equation after 200 time steps, on a $64 \times 64 \times 64$ lattice, with the parameters $\alpha=0.5, \beta=-1$. In the figure, an amplitude isosurface is imaged, with shadings drawn from the phase values along the isosurface. In this way, a number of vortex tubes appear, each surrounding a vortex line of zero amplitude. The shading indicates a twist in the phase field around each vortex line.

To perform a study analogous to the present two-dimensional one, we need an analog of the vortex density in three dimensions. A natural choice is the total length of the vortex lines. Furthermore, we can think of our two-dimensional system as simply a planar cross-section of the three-dimensional system - vortices and antivortices are then just slices through vortex lines. Since a vortex line can thread 


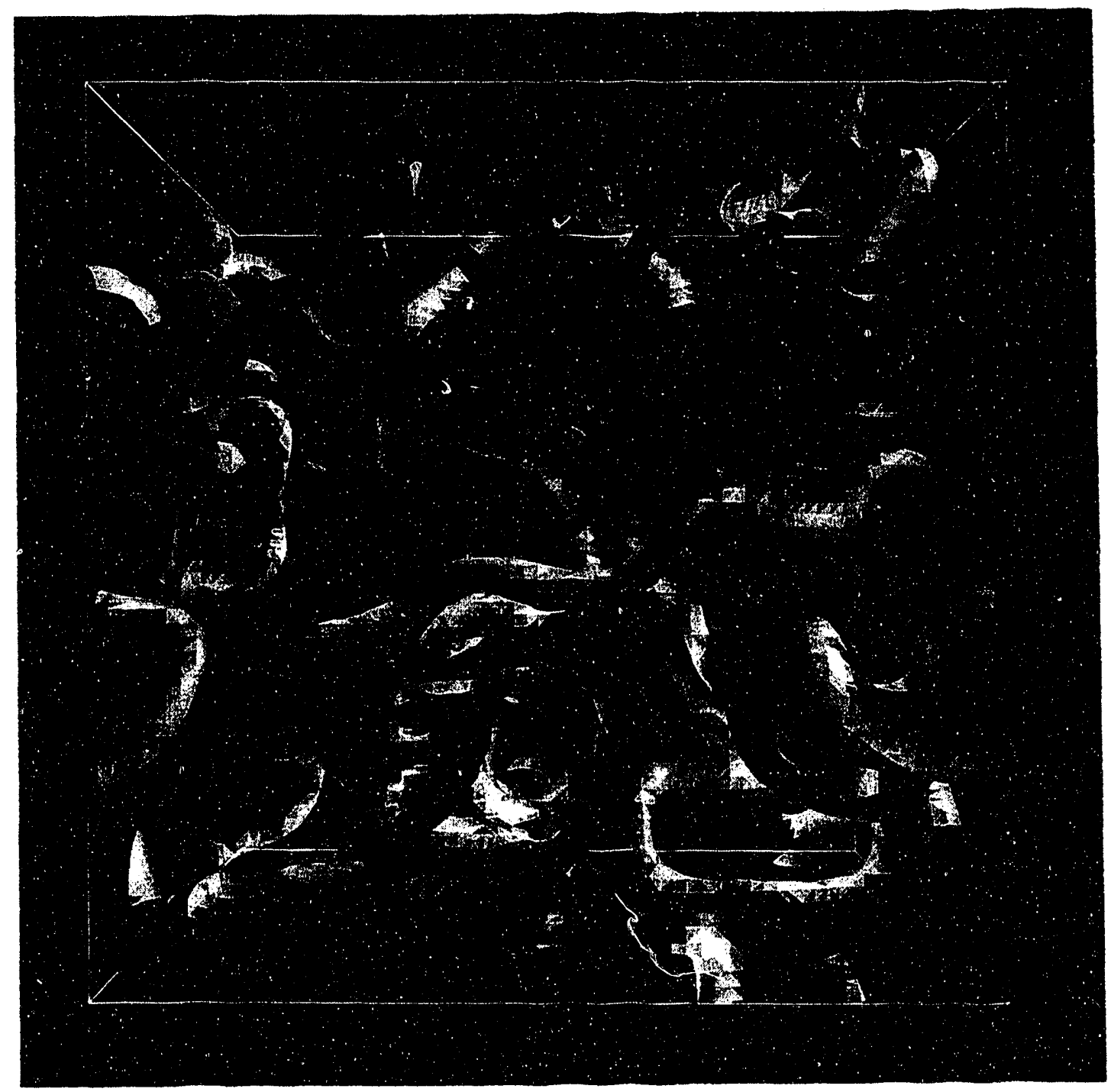

Figure 5.3: Vortex tubes in three dimensions, at $t=200$. An amplitude isosurface of $|A|=0.15$ is displayed, shaded by the phase value at each point of the isosurface. Parameters are $\alpha=0.5$, bet $a=-1$; system size is $64 \times 64 \times 64$. 
through this plane more than once, vortex-antivortex creation and annihitation is then just the passage of a vortex hairpin into the plane and out of the plane, respectively. It would be very interesting study the corresponding turbulent phase of the vortex lines, since now only the complex motions of vortex lines are necessary: to produce it. 


\section{Bibliography}

[1] B. P. Belousov, in Oscillations and Traveling Waves in Chemical Systems, edited by R. Field and M. Burger (Wiley, New York, 1985).

[2] A. M. Zhabotinskii Biofizika 9 (3) 306 (1964).

[3] A. N. Zaikin and A. M. Zhabotinskii Nature 225535 (1970).

[4] A. M. Zhabotinskii, in Oscillations and Traveling Waves in Chemical Systems. edited by R. Field and M. Burger (Wiley, New Vork, 1985).

[5] J. J. Tyson The Belousov-Zhabotinskii Reaction (Springer-Verlag, Berlin. 1976).

[6] K. Kaneko Prog. Theor. Phys. 741033 (1985).

[7] T. Bohr and O. B. Christensen Phys. Rev. Lett. 632161 (1989).

[8] D. Stassinopoulos. G. Huber and P. Alstrøm Phys. Rev. Lett. 643007 (1990).

[9] E. Hopf Ber. Math.-Phys. Ki. Sächs. Akad. Wiss. Leipzig 941 (1912).

[10] G. looss and D. D. Joseph Elementary Stability and Bifurcation Theory (Springer-Verlag. New York, 1980). 
[11] M. Golubitsky and D. C. Schaeffer Singularitits and (iroups.s in Bifurculion Theory (Springer-Verlag, New York, 1985).

[12] A. C. Newell and J. A. Whitehead J. Fluid Mech. 38279 (1969).

[13] Y. Kuramoto Chemical Oscillations, Waves, and Turbulence (SpringerVerlag, Berlin, 1984).

[14] Y. Oono and S. Puri Phys. Rev. Lett. 58, 836 (1987).

[15] T. Bohr, A. W. Pedersen, M. H. Jensen and D. A. Rand, in New Trends in Nonlinear Dynamics and Pattern Forming Phenomenn: The Cieometry of Nonequilibrium, edited by P. Coullet and P. Huerre (Plenum. New York. 1989).

[16] T. Bohr, A. W. Pedersen, M. H. Jensen and D. A. Rand, in Nonlinear Evolution of Spatio-Temporal Structures in Dissipative Continuous Systems, edited by F. Busse and L. Kramer (Plenum, New York, 1990).

[17] T. B. Benjamin and J. E. Feir J. Fluid Mech. 27417 (1967).

[18] G. Huber, P. Alstrøm and T. Bohr Phys. Rev. Lett. 692380 (1992).

[19] P. Glansdorff and I. Prigogine Thermodynamic Theory of Structure, Stability and Fluctuations (Wiley, London, 1971).

[20] 1. Prigogine From Being to Becoming (Freeman. San Francisco. 1980).

[21] I. Prigogine and R. Lefever J. Chem. Phys. 48 1695 (1968).

[22] P. Coullet. L. Gil and J. Lega Phys. Rev. Lett. 621619 (1989). 
[23] E. Bodenschatz, M. Kaiser, L. Kramer, W. Pesch, A. Weber and W. Zimmermann, in New Trends in Nonlinear Dynamics and Pattern Forming Phenomena: The Geometry of Nonequilibrium, edited by P. Coullet and P. Huerre (Plenum, New York, 1989).

[24] T. Bohr, A. W. Pedersen and M. H. Jensen Phys. Rev. A 423626 (1990).

[25] A. T. Winfree When Time Breaks Down (Princeton, Princeton, 198i).

[26] C. Elphick and E. Meron, preprint (1989).

[27] L. Gil, J. Lega and J. L. Meunier Phys. Rev. A 411138 (1990).

[28] I. S. Aranson, L. B. Aranson, L. Kramer and A. Weber, preprint (1991); A. Weber, L. Kramer, I. S. Aranson and L. B. Aranson, preprint (1991).

[29] J. D. Gunton, M. San Miguel and P. S. Sahni, in Phase Transitions and Critical Phenomena, vol. 8, edited by C. Domb and J. L. Lebowitz (Academic, New York, 1983).

[30] R. Loft and T. A. DeGrand Phys. Rev. B 358528 (1987).

[31] H. Toyoki Phys. Rev. A 42911 (1990).

[32] M. Mondello and N. Goldenfeld Phys. Rev. A 425865 (19y0).

[33] I. M. Lifshitz Sov. Phys. JETP 15939 (1962).

[34] S. M. Allen and J. W: Cahn Acta Metall. 271085 (1979).

[35] S. F. Burlatskii Teor. Eksp. Kihim. 14483 (1978).

[36] F. Leyvraz and S. Redner Phys. Rev. Leit. 662168 (1991). 
[37] J. M. Kosterlitz and D. J. Thouless J. Phys. C: Solid St. Phys. 5 1:1 (1972).

[38] D. Dhar Phys. Lett. A 8119 (1981).

[39] J. C. McWilliams J. Fluid Mech. 219361 (1990).

[40] G. F. Carnevale. J. C. McWilliams, Y. Pomeau, J. B. Weiss and W. R. Young Phys. Rev. Lett. 662735 (1991).

[41] J. B. Weiss and J. C. McWilliams Phys. Fluids A (submitted).

[42] P. Tabeling, S. Burkhart, O. Cardoso and H. Willaime Phys. Rev. Lett. 67 $3772(1991)$.

[43] W. Eckhaus Studies in Non-linear Stability Theory (Springer, New York, 1965).

[44] T. Bohr, personal communication.

[45] N. Kopell and L. N. Howard Stud. Appl. Math. 52291 (1973).

[46] Y'. Kuramoto and T. Tsuzuki Prog. Theor. Phys. 55356 (1976).

[47] Y. Kuramoto Suppl. Prog. Theor. Phys. 64346 (1978).

[48] P. Minnhagen Rev. Mod. Phys. 591001 (1987).

[49] X.-G. Wu and R. Kapral J. Chem. Phys. 941411 (1991). 

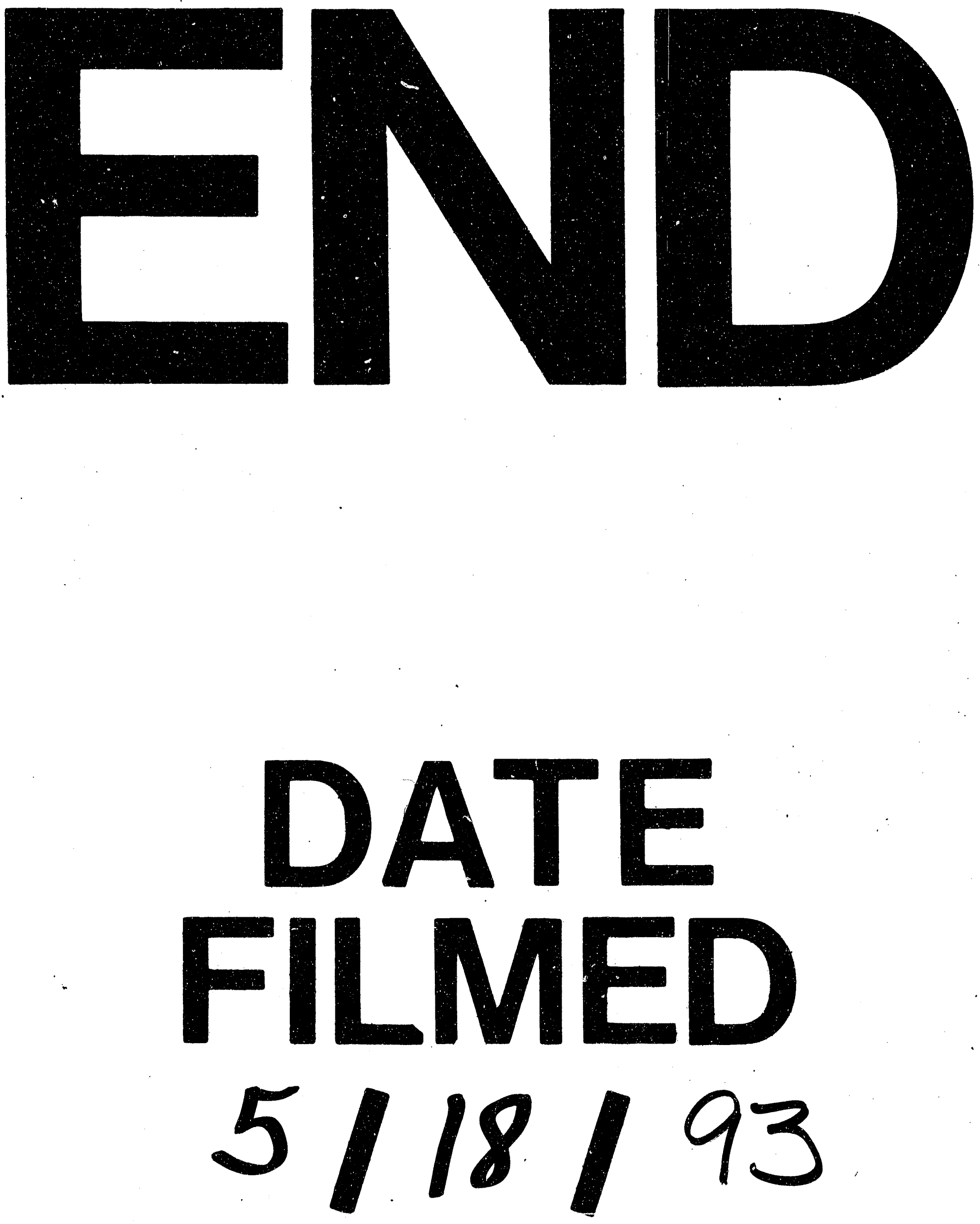
